\title{
Asymmetric Friedel-Crafts Reaction of Indoles with Imines by an Organic Catalyst
}

\author{
Yong-Qiang Wang, Jun Song, Ran Hong, Hongming Li and Li Deng* \\ Department of Chemistry \\ Brandeis University \\ Waltham, Massachusetts 02454-9110. \\ *To whom correspondence should be addressed
}

\section{Supporting Information}

General Information. ${ }^{1} \mathrm{H}$ and ${ }^{13} \mathrm{C}$ NMR spectra were recorded on a Varian instrument (400 $\mathrm{MHz}$ and $100 \mathrm{MHz}$, respectively) and internally referenced to tetramethylsilane signal or residual protio solvent signals. Data for ${ }^{1} \mathrm{H}$ NMR are recorded as follows: chemical shift $(\delta$, ppm), multiplicity ( $\mathrm{s}$, singlet; $\mathrm{d}$, doublet; $\mathrm{t}$, triplet; q, quartet; $\mathrm{m}$, multiplet), intergration, coupling constant $(\mathrm{Hz})$. Data for ${ }^{13} \mathrm{C} \mathrm{NMR}$ are reported in terms of chemical shift $(\delta, \mathrm{ppm})$. Infrared spectra were recorded on a Perkin Elmer FT-IR Spectrometer and are reported in frequency of absorption. Low resolution mass spectra for all the new compounds were performed by 70SE EI+, and were recorded and exact mass spectra on a 70-VSE-B high resolution mass spectrometer. Specific rotations were measured on a Jasco Digital Polarimeter.

High performance liquid chromatography (HPLC) analysis was performed on a HewlettPackard 1100 Series instrument equipped with a quaternary pump, using a Daicel Chiralcel OD Column (250 x $4.6 \mathrm{~mm})$. UV absorption was monitored at $220 \mathrm{~nm}$ or at $254 \mathrm{~nm}$.

Materials: $N$-sulfonyl imines $\mathbf{2 b},{ }^{1} \mathbf{2} \mathbf{c},{ }^{2} \mathbf{2 d - 2 f},{ }^{1} \mathbf{2 g},{ }^{3} \mathbf{2 h}-\mathbf{2} \mathbf{l}^{1}$ were prepared according to literature procedures. $N$-sulfonyl imine 2a and indoles $\mathbf{1 A - 1 F}$, were purchased from Aldrich Inc. and used without further purification. Cinchona alkaloids QD-4a-c, ${ }^{4} \mathbf{5}, 6$ and $7^{5}$ were prepared according to literature procedures (QD-4a-c, 5, 6 were prepared from quinidine, and 7 was prepared from quinine). 

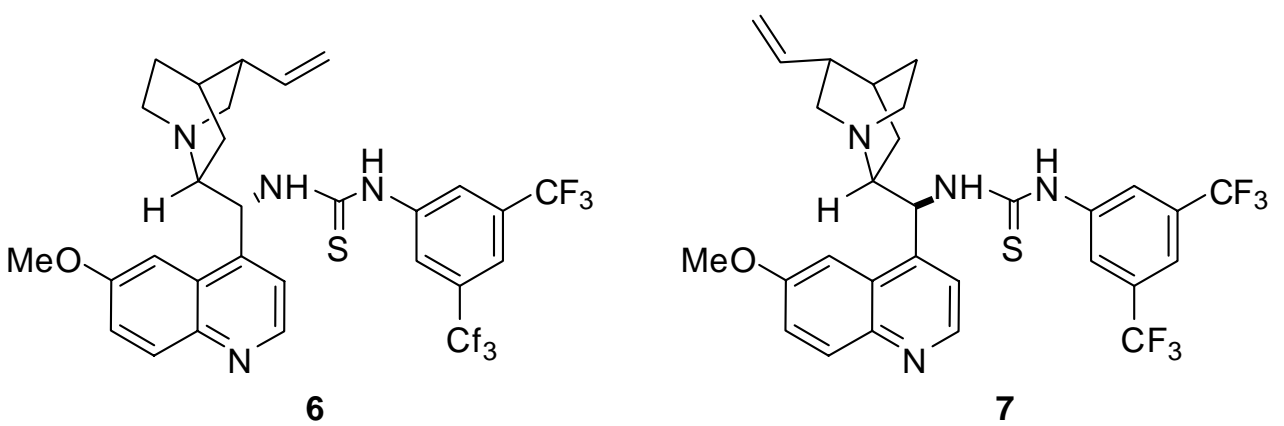

\section{Preparation of $\mathrm{N}$-sulfonyl imine $\mathbf{2 m}$ :}

A mixture of Benzyloxyacetaldehyde (1.502 g, $10.0 \mathrm{mmol}), p$-Toluenesulfonamide $(1.712 \mathrm{~g}, 10.0 \mathrm{mmol})$, sodium $p$-Tolenesulfinate $(1.782 \mathrm{~g}, 10.0 \mathrm{mmol})$ in formic acid ( $95 \%, 15 \mathrm{~mL})$ and $\mathrm{H}_{2} \mathrm{O}(15 \mathrm{~mL})$ was stirred for $24 \mathrm{~h}$ at room temperature. The resulting white precipitate was collected by filtration, washed with $\mathrm{H}_{2} \mathrm{O}(3 \times 10 \mathrm{~mL})$, and hexane ( $2 \times 10 \mathrm{~mL})$, then dissolved in $\mathrm{CH}_{2} \mathrm{Cl}_{2}(100 \mathrm{~mL})$, followed by addition of $\mathrm{H}_{2} \mathrm{O}(35 \mathrm{~mL})$ and Sat. aq $\mathrm{NaHCO}_{3}(35 \mathrm{~mL})$. The solution was well stirred for $10 \mathrm{~min}$ at room temperature. The organic phase was collected, the aqueous phase was extracted with $\mathrm{CH}_{2} \mathrm{Cl}_{2}(3 \times 70 \mathrm{~mL})$. The combined organic layers were dried over $\mathrm{MgSO}_{4}$, concentrated to yield $N$-sulfonyl imine $\mathbf{2 m}$ as a colorless oil $(2.128 \mathrm{~g}, 70 \%)$. This material was used without further purification. ${ }^{1} \mathrm{H}$ NMR $\left(400 \mathrm{MHz}, \mathrm{CDCl}_{3}\right) \delta 2.45(\mathrm{~s}, 3 \mathrm{H}), 4.35(\mathrm{~d}, J=3.2$ Hz, 2H), 4.60 (s, 2 H), 7.28-7.39 (m, 7H), 7.81 (d, $J=8.4 \mathrm{~Hz}, 2 \mathrm{H}), 8.56$ (t, $J=2.8 \mathrm{~Hz}$, $1 \mathrm{H}) ;{ }^{13} \mathrm{C}$ NMR $\left(100 \mathrm{MHz}, \mathrm{CDCl}_{3}\right) \delta 22.4,71.0,74.4,128.8,129.0,129.1,129.3,130.6$, 134.6, 137.2, 145.8, 175.8; IR (neat) v 3282, 2922, 1644, 1324, 1163, 1091, $680 \mathrm{~cm}^{-1}$; HRMS: calcd for $\left(\mathrm{M}^{+}\right) \mathrm{C}_{16} \mathrm{H}_{17} \mathrm{NO}_{3} \mathrm{~S}: 303.0929$; found 303.0928 .

General procedure for asymmetric addition of indoles to $\mathrm{N}$-Sulfonyl imines:<smiles>[R]C=[NH2+]</smiles>

2

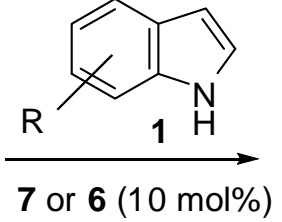

EtOAc, $50{ }^{\circ} \mathrm{C}$

8-72 h

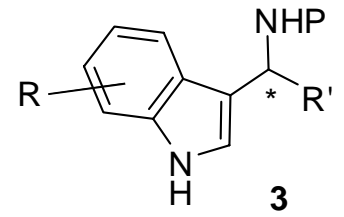

3

To a mixture of $N$-sulfonyl imines $2(0.3 \mathrm{mmol}), 7$ or $6(10 \mathrm{~mol} \%)$ in EtOAc $(0.15 \mathrm{~mL})$ was added indoles $(0.6 \mathrm{mmol})$ in one portion. The resulting mixture was heated to $50{ }^{\circ} \mathrm{C}$ and kept at $50{ }^{\circ} \mathrm{C}$ for $8-72$ hours (as indicated in Tables 2 and 3). The reaction mixture 
was subjected to flash chromatography to afford the desired product 3. For entry 15 in Table 2, the reactions were carried out at $70{ }^{\circ} \mathrm{C}$. For entry 5 in Table $3,0.30 \mathrm{~mL}$ of toluene was used, and the reactions were carried out at $-25^{\circ} \mathrm{C}$.

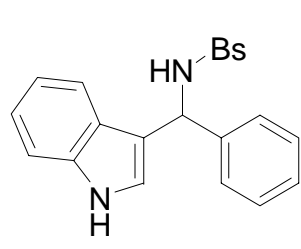

(-)-3Aa This product was obtained as a green foam in $87 \%$ yield after flash chromatography (silica gel: Ethyl acetate / Hexanes $=1 / 3$ ) and in $94 \%$ ee as determined by HPLC [Daicel Chiralcel OD, Hexanes / $\mathrm{IPA}=60 / 40,1.0 \mathrm{ml} \cdot \mathrm{min}^{-1}, \lambda=254 \mathrm{~nm}, \mathrm{t}$ (major) $=11.5 \mathrm{~min}, \mathrm{t}$ $(\operatorname{minor})=7.5 \mathrm{~min}]$ from a reaction catalyzed by $7(10 \mathrm{~mol} \%)$ at $50{ }^{\circ} \mathrm{C}$ for $48 \mathrm{~h} .[\alpha]_{\mathrm{D}}{ }^{25}=-$ 17.9 (c 1.01, Acetone); ${ }^{1} \mathrm{H}$ NMR (400 MHz, $\left.\mathrm{CDCl}_{3}\right) \delta 5.34$ (d, $\left.J=7.2 \mathrm{~Hz}, 1 \mathrm{H}\right), 5.85$ (d, $J$ $=7.2 \mathrm{~Hz}, 1 \mathrm{H}), 6.56(\mathrm{~s}, 1 \mathrm{H}), 6.98(\mathrm{t}, J=7.6 \mathrm{~Hz}, 1 \mathrm{H}), 7.08-7.20(\mathrm{~m}, 6 \mathrm{H}), 7.21-7.30(\mathrm{~m}$, 4H). $7.40(\mathrm{t}, J=7.4 \mathrm{~Hz}, 1 \mathrm{H}), 7.62(\mathrm{~d}, J=8.0 \mathrm{~Hz}, 2 \mathrm{H}), 7.98(\mathrm{br}, 1 \mathrm{H}) ;{ }^{13} \mathrm{C}$ NMR $(100$ $\left.\mathrm{MHz}, \mathrm{CDCl}_{3}\right) \delta 55.0,111.3,116.1,119.2,119.9,122.4,123.9,125.3,127.0,127.1$, 127.4, 128.3, 128.6, 132.2, 136.5, 140.0, 140.3; IR (neat) v 1457, 1447, 1158; HRMS: calcd for $\left(\mathrm{M}+\mathrm{NH}_{4}{ }^{+}\right) \mathrm{C}_{21} \mathrm{H}_{22} \mathrm{~N}_{3} \mathrm{O}_{2} \mathrm{~S}: 380.1433$; found 380.1435 .

$(+)-3$ Aa This product was obtained as a green foam in $96 \%$ yield and $92 \%$ ee from a reaction catalyzed by $6(10 \mathrm{~mol} \%)$ at $50{ }^{\circ} \mathrm{C}$ for $48 \mathrm{~h}$.

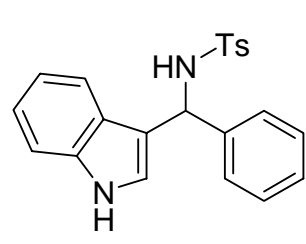

(-)-3Ab This product was obtained as a colorless solid in 96\% yield after flash chromatography (silica gel: Ethyl acetate / Hexanes $=1 / 2$ ) and in $94 \%$ ee as determined by HPLC [Daicel Chiralcel OD, Hexanes $/ \mathrm{IPA}=60 / 40,1.0 \mathrm{ml} \cdot \mathrm{min}^{-1}, \lambda=220 \mathrm{~nm}, \mathrm{t}$ (major) $=10.9$ min, $\mathrm{t}($ minor $)=6.9 \mathrm{~min}]$ from a reaction catalyzed by $7(10 \mathrm{~mol} \%)$ at $50{ }^{\circ} \mathrm{C}$ for $60 \mathrm{~h}$; $[\alpha]_{\mathrm{D}}^{25}=-17.0\left(c 1.0, \mathrm{CHCl}_{3}\right)$; m. p. $73-75{ }^{\circ} \mathrm{C} ;{ }^{1} \mathrm{H} \mathrm{NMR}\left(400 \mathrm{MHz}, \mathrm{CDCl}_{3}\right) \delta 2.34(\mathrm{~s}$, $3 \mathrm{H}), 4.98(\mathrm{~d}, J=7.2 \mathrm{~Hz}, 1 \mathrm{H}), 5.82(\mathrm{~d}, J=7.2 \mathrm{~Hz}, 1 \mathrm{H}), 6.61(\mathrm{~s}, 1 \mathrm{H}), 6.97$ (t, $J=7.6 \mathrm{~Hz}$, $1 \mathrm{H}), 7.05$ (d, $J=8.0 \mathrm{~Hz}, 2 \mathrm{H}), 7.08-7.29(\mathrm{~m}, 8 \mathrm{H}), 7.52$ (d, $J=8.0 \mathrm{~Hz}, 2 \mathrm{H}), 8.02(\mathrm{br}, 1 \mathrm{H})$; ${ }^{13} \mathrm{C}$ NMR $\left(100 \mathrm{MHz}, \mathrm{CDCl}_{3}\right) \delta 22.1,55.7,112.0,116.9,119.9,120.5,123.1,124.5$, $126.0,127.8,127.9,128.0,129.0,129.9,137.2,138.0,140.6,140.9,143.7$; IR (neat) $v$ 
1456, 1320, 1157, 1093, 743, $668 \mathrm{~cm}^{-1}$; HRMS: calcd for $\left(\mathrm{M}+\mathrm{NH}_{4}{ }^{+}\right) \mathrm{C}_{22} \mathrm{H}_{24} \mathrm{~N}_{3} \mathrm{O}_{2} \mathrm{~S}$ : 394.1589; found 394.1589.

(+)-3Ab This product was obtained as a colorless solid in $96 \%$ yield and $94 \%$ ee from a reaction catalyzed by $6(10 \mathrm{~mol} \%)$ at $50{ }^{\circ} \mathrm{C}$ for $60 \mathrm{~h}$.

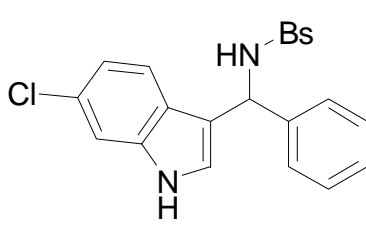

(-)-3Ba This product was obtained as a pink solid in $86 \%$ yield after flash chromatography (silica gel: Ethyl acetate / Hexanes $=1$ /3) and in $90 \%$ ee as determined by HPLC [Daicel Chiralcel OD, Hexanes $/ \mathrm{IPA}=70 / 30,1.0 \mathrm{ml} \cdot \mathrm{min}^{-1}, \lambda=254 \mathrm{~nm}, \mathrm{t}$ (major) $=8.2$ min, $\mathrm{t}($ minor $)=6.1 \mathrm{~min}]$ from a reaction catalyzed by $7(10 \mathrm{~mol} \%)$ at $50{ }^{\circ} \mathrm{C}$ for $72 \mathrm{~h} ; \mathrm{m}$. p. $182-183{ }^{\circ} \mathrm{C}$; ${ }^{1} \mathrm{H}$ NMR $\left(400 \mathrm{MHz}, \mathrm{CD}_{3} \mathrm{COCD}_{3}\right) \delta 5.92(\mathrm{~d}, J=8.4 \mathrm{~Hz}, 1 \mathrm{H}), 6.85$ (s, $1 \mathrm{H}), 6.93(\mathrm{~d}, J=8.4 \mathrm{~Hz}, 1 \mathrm{H}), 7.14-7.22(\mathrm{~m}, 3 \mathrm{H}), 7.26-7.40(\mathrm{~m}, 7 \mathrm{H}), 7.47$ (t, $J=7.6 \mathrm{~Hz}$, $1 \mathrm{H}), 7.70(\mathrm{~d}, J=7.2 \mathrm{~Hz}, 2 \mathrm{H}), 10.23(\mathrm{br}, 1 \mathrm{H}) ;{ }^{13} \mathrm{C} \mathrm{NMR}\left(100 \mathrm{MHz}, \mathrm{CD}_{3} \mathrm{COCD}_{3}\right) \delta 55.1$, 111.4, 116.7, 119.6, 120.7, 124.9, 125.4, 127.0, 127.2, 127.3, 127.5, 128.3, 128.8, 132.0, 137.6, 141.4, 142.2; IR (neat) $\vee$ 1448, 1318, 1159; HRMS: calcd for $\left(\mathrm{M}+\mathrm{NH}_{4}{ }^{+}\right)$ $\mathrm{C}_{21} \mathrm{H}_{21} \mathrm{~N}_{3} \mathrm{O}_{2} \mathrm{SCl}$ : 414.1043; found 414.1031.

$(+)-3 B a$ This product was obtained as a pink solid in $88 \%$ yield and $95 \%$ ee from a reaction catalyzed by $6(10 \mathrm{~mol} \%)$ at $50{ }^{\circ} \mathrm{C}$ for $72 \mathrm{~h} .[\alpha]_{\mathrm{D}}{ }^{25}=+10.3(c 1.04$, Acetone).

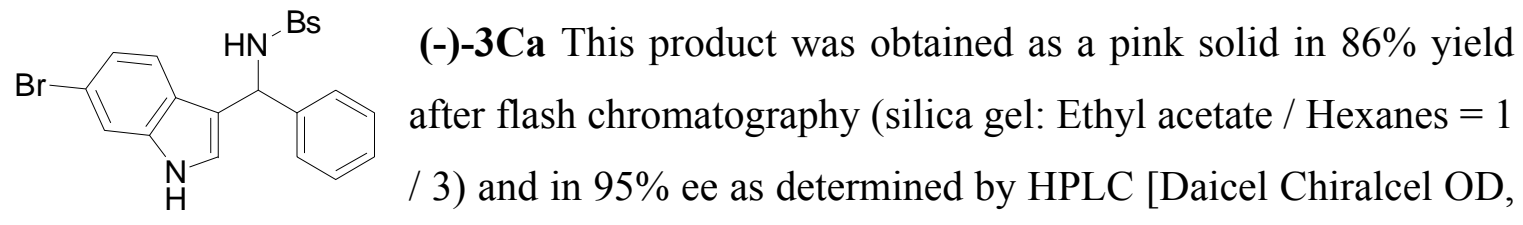
Hexanes $/ \mathrm{IPA}=60 / 40,1.0 \mathrm{ml} \cdot \mathrm{min}^{-1}, \lambda=254 \mathrm{~nm}, \mathrm{t}$ (major) $=7.3 \mathrm{~min}, \mathrm{t}($ minor $)=5.7$ $\mathrm{min}]$ from a reaction catalyzed by $7(10 \mathrm{~mol} \%)$ at $50{ }^{\circ} \mathrm{C}$ for $60 \mathrm{~h}$. $[\alpha]_{\mathrm{D}}{ }^{25}=-19.8(c \mathrm{c} 1.04$, Acetone); m. p. $172-173{ }^{\circ} \mathrm{C} ;{ }^{1} \mathrm{H}$ NMR $\left(400 \mathrm{MHz}, \mathrm{CD}_{3} \mathrm{COCD}_{3}\right) \delta 5.92(\mathrm{~d}, J=8.4 \mathrm{~Hz}$, $1 \mathrm{H}), 6.82(\mathrm{~s}, 1 \mathrm{H}), 7.04(\mathrm{dd}, J=2.0,8.8 \mathrm{~Hz}, 1 \mathrm{H}), 7.10-7.22(\mathrm{~m}, 3 \mathrm{H}), 7.24-7.36(\mathrm{~m}, 6 \mathrm{H})$. $7.44(\mathrm{t}, J=7.2 \mathrm{~Hz}, 1 \mathrm{H}), 7.54$ (s, 1H), $7.68(\mathrm{~d}, J=7.6 \mathrm{~Hz}, 2 \mathrm{H}), 10.21$ (br, $1 \mathrm{H}) ;{ }^{13} \mathrm{C} \mathrm{NMR}$ $\left(100 \mathrm{MHz}, \mathrm{CD}_{3} \mathrm{COCD}_{3}\right) \delta 55.0,114.4,115.0,116.6,121.0,122.2,125.1,125.3,127.0$, 
127.1, 127.5, 128.3, 128.7, 132.0, 138.0, 141.3, 142.1; IR (neat) $\vee 1448,1318,1158$; HRMS: calc'd for $\left(\mathrm{M}+\mathrm{NH}_{4}{ }^{+}\right) \quad \mathrm{C}_{21} \mathrm{H}_{21} \mathrm{~N}_{3} \mathrm{O}_{2} \mathrm{SBr}$ : 458.0529; found 458.0538 .

$(+)-3 \mathrm{Ca}$ This product was obtained as a pink solid in $88 \%$ yield and $95 \%$ ee from a reaction catalyzed by $6(10 \mathrm{~mol} \%)$ at $50{ }^{\circ} \mathrm{C}$ for $72 \mathrm{~h}$.

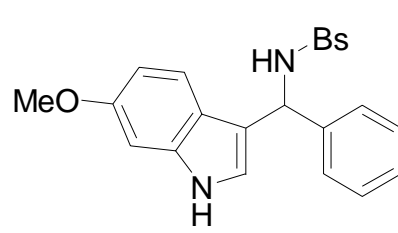

(-)-3Da This product was obtained as a gray foam in $89 \%$ yield after flash chromatography (silica gel: Ethyl acetate / Hexanes = $1 / 3$ ) and in $92 \%$ ee as determined by HPLC [Daicel Chiralcel $\mathrm{OD}$, Hexanes $/ \mathrm{IPA}=60 / 40,1.0 \mathrm{ml} \cdot \mathrm{min}^{-1}, \lambda=254 \mathrm{~nm}, \mathrm{t}$ $($ major $)=9.7 \mathrm{~min}, \mathrm{t}(\operatorname{minor})=6.7 \mathrm{~min}]$ from a reaction catalyzed by $7(10 \mathrm{~mol} \%)$ at 50 ${ }^{\circ} \mathrm{C}$ for $36 \mathrm{~h} .[\alpha]_{\mathrm{D}}{ }^{25}=-19.2\left(c 1.01\right.$, Acetone); ${ }^{1} \mathrm{H}$ NMR $\left(400 \mathrm{MHz}, \mathrm{CDCl}_{3}\right) \delta 3.74(\mathrm{~s}, 3 \mathrm{H})$, $5.88(\mathrm{~d}, J=7.6 \mathrm{~Hz}, 1 \mathrm{H}), 6.59(\mathrm{dd}, J=2.4,8.4 \mathrm{~Hz}, 1 \mathrm{H}), 6.63(\mathrm{~d}, J=2.0 \mathrm{~Hz}, 1 \mathrm{H}), 6.88(\mathrm{~d}$, $J=2.0 \mathrm{~Hz}, 1 \mathrm{H}), 7.08-7.20(\mathrm{~m}, 5 \mathrm{H}), 7.33(\mathrm{t}, J=8.0 \mathrm{~Hz}, 4 \mathrm{H}), 7.44(\mathrm{t}, J=7.6 \mathrm{~Hz}, 1 \mathrm{H}), 7.70$ (d, $J=8.0 \mathrm{~Hz}, 1 \mathrm{H}), 9.84(\mathrm{br}, 1 \mathrm{H}) ;{ }^{13} \mathrm{C} \mathrm{NMR}\left(100 \mathrm{MHz}, \mathrm{CDCl}_{3}\right) \delta 55.0,55.4,94.6,109.4$, $116.3,120.0,120.5,122.9,127.0,127.1,127.5,128.2,128.7,132.0,138.0,141.7,142.2$, 156.7; IR (neat) v 1448, 1307, 1160; HRMS: calcd for $\left(\mathrm{M}+\mathrm{H}^{+}\right) \mathrm{C}_{22} \mathrm{H}_{21} \mathrm{~N}_{2} \mathrm{O}_{2} \mathrm{~S}$ : 393.1273; found 393.1247 .

(+)-3Da This product was obtained as a gray solid in $90 \%$ yield and $93 \%$ ee from a reaction catalyzed by $6(10 \mathrm{~mol} \%)$ at $50{ }^{\circ} \mathrm{C}$ for $36 \mathrm{~h}$.

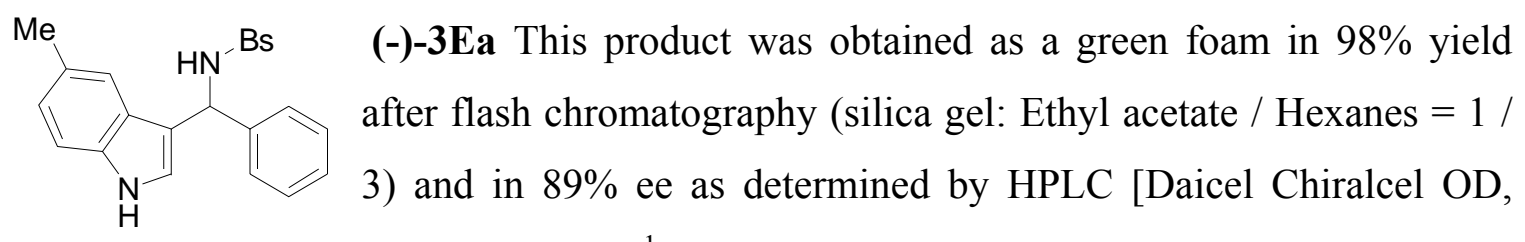
Hexanes $/ \mathrm{IPA}=60 / 40,1.0 \mathrm{ml} \cdot \mathrm{min}^{-1}, \lambda=254 \mathrm{~nm}, \mathrm{t}$ (major) $=12.0 \mathrm{~min}, \mathrm{t}($ minor $)=5.7$ $\mathrm{min}$ ] from a reaction catalyzed by $7(10 \mathrm{~mol} \%)$ at $50{ }^{\circ} \mathrm{C}$ for $48 \mathrm{~h} .[\alpha]_{\mathrm{D}}{ }^{25}=-19.2(c 1.03$, Acetone); ${ }^{1} \mathrm{H}$ NMR (400 MHz, $\left.\mathrm{CD}_{3} \mathrm{OD}\right) \delta 2.29$ (s, 3H), 5.79 (s, 1H), $6.54(\mathrm{~s}, 1 \mathrm{H}), 6.87$ (d, $J=8.0 \mathrm{~Hz}, 1 \mathrm{H}), 6.99$ (s, 1H), 7.08-7.20 (m, 4H), 7.20-7.32 (m, 4H), 7.39 (t, $J=7.6$ $\mathrm{Hz}, 1 \mathrm{H}), 7.65(\mathrm{dd}, J=1.2,8.8 \mathrm{~Hz}, 2 \mathrm{H}) ;{ }^{13} \mathrm{C}$ NMR $\left(100 \mathrm{MHz}, \mathrm{CD}_{3} \mathrm{OD}\right) \delta 21.8,56.3$, 112.1, 116.4, 119.7, 124.4, 125.4, 127.5, 128.08, 128.12, 128.6, 129.16, 129.20, 129.7, 
133.2, 136.7, 142.8, 142.9; IR (neat) $\vee 1448,1322,1159$; HRMS: calcd for $\left(\mathrm{M}+\mathrm{NH}_{4}{ }^{+}\right)$ $\mathrm{C}_{22} \mathrm{H}_{24} \mathrm{~N}_{3} \mathrm{O}_{2}$ : 394.1572; found 394.1589.

$(+)-3-E a$ This product was obtained as a green foam in $90 \%$ yield and $91 \%$ ee from a reaction catalyzed by $6(10 \mathrm{~mol} \%)$ at $50{ }^{\circ} \mathrm{C}$ for $48 \mathrm{~h}$.

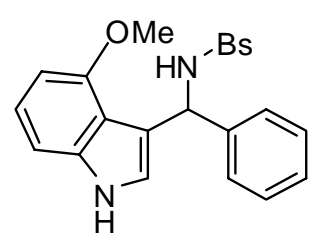

(-)-3Fa This product was obtained as a white solid in $88 \%$ yield after flash chromatography (silica gel: Ethyl acetate / Hexanes $=1 / 3$ ) and in $91 \%$ ee as determined by HPLC [Daicel Chiralcel OD, Hexanes / $\mathrm{IPA}=70 / 30,1.0 \mathrm{ml} \cdot \mathrm{min}^{-1}, \lambda=254 \mathrm{~nm}, \mathrm{t}$ (major) $=8.3 \mathrm{~min}, \mathrm{t}$ $($ minor $)=6.6 \mathrm{~min}]$ from a reaction catalyzed by $7(10 \mathrm{~mol} \%)$ at $50{ }^{\circ} \mathrm{C}$ for $72 \mathrm{~h} .[\alpha]_{\mathrm{D}}{ }^{25}=-$ 31.5 (c 1.25, Acetone); m. p. 61-62 ${ }^{\circ} \mathrm{C}$; ${ }^{1} \mathrm{H}$ NMR (400 MHz, $\left.\mathrm{CD}_{3} \mathrm{COCD}_{3}\right) \delta 3.73$ (s, $3 \mathrm{H}), 6.10(\mathrm{~d}, J=9.2 \mathrm{~Hz}, 1 \mathrm{H}), 6.43(\mathrm{~d}, J=7.2 \mathrm{~Hz}, 1 \mathrm{H}), 6.90$ (t, $J=8.8 \mathrm{~Hz}, 2 \mathrm{H}), 6.96(\mathrm{t}, J$ $=8.0 \mathrm{~Hz}, 1 \mathrm{H}), 7.18(\mathrm{t}, J=7.6 \mathrm{~Hz}, 4 \mathrm{H}), 7.25-7.32(\mathrm{~m}, 3 \mathrm{H}), 7.55-7.64(\mathrm{~m}, 2 \mathrm{H}), 10.03(\mathrm{br}$, $1 \mathrm{H}) ;{ }^{13} \mathrm{C} \mathrm{NMR}\left(100 \mathrm{MHz}, \mathrm{CD}_{3} \mathrm{COCD}_{3}\right) \delta 54.8,55.4,99.9,105.3,115.3,115.9,122.7$, $122.9,126.6,126.8,127.3,128.0,128.3,131.6,138.8,142.0,143.56,153.4$; IR (neat) $v$ 3386, 1324, 1159; HRMS: calc'd for $\left(\mathrm{M}+\mathrm{NH}_{4}{ }^{+}\right) \mathrm{C}_{22} \mathrm{H}_{24} \mathrm{~N}_{3} \mathrm{O}_{3} \mathrm{~S}$ : 410.1548; found 410.1538 .

$(+)-3 F a$ This product was obtained as a white solid in $94 \%$ yield and $89 \%$ ee from a reaction catalyzed by $6(10 \mathrm{~mol} \%)$ at $50{ }^{\circ} \mathrm{C}$ for $72 \mathrm{~h}$.

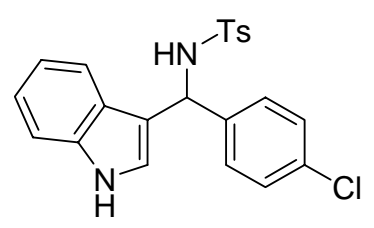

(-)-3Ac This product was obtained as a colorless solid in $98 \%$ yield after flash chromatography (silica gel: Ethyl acetate / Hexanes $=1 / 2$ ) and in $94 \%$ ee as determined by HPLC [Daicel Chiralcel OD, Hexanes $/ \mathrm{IPA}=60 / 40,1.0 \mathrm{ml} \cdot \mathrm{min}^{-1}, \lambda=220 \mathrm{~nm}$, $\mathrm{t}($ major $)=11.6 \mathrm{~min}, \mathrm{t}($ minor $)=7.5 \mathrm{~min}]$ from a reaction catalyzed by $7(10 \mathrm{~mol} \%)$ at 50 ${ }^{\circ} \mathrm{C}$ for $28 \mathrm{~h} ;[\alpha]_{\mathrm{D}}{ }^{25}=-16.8\left(c 1.0, \mathrm{CHCl}_{3}\right)$; m. p. $183-184{ }^{\circ} \mathrm{C} ;{ }^{1} \mathrm{H}$ NMR $(400 \mathrm{MHz}$, $\left.\mathrm{CDCl}_{3}\right) \delta 2.37(\mathrm{~s}, 3 \mathrm{H}), 5.29(\mathrm{~d}, J=7.6 \mathrm{~Hz}, 1 \mathrm{H}), 5.78(\mathrm{~d}, J=7.6 \mathrm{~Hz}, 1 \mathrm{H}), 6.59(\mathrm{~s}, 1 \mathrm{H})$, $6.99(\mathrm{t}, J=8.0 \mathrm{~Hz}, 1 \mathrm{H}), 7.05-7.31(\mathrm{~m}, 9 \mathrm{H}), 7.52(\mathrm{~d}, J=8.8 \mathrm{~Hz}, 2 \mathrm{H}), 8.04(\mathrm{br}, 1 \mathrm{H}) ;{ }^{13} \mathrm{C}$ 
NMR (100 MHz, $\left.\mathrm{CDCl}_{3}\right) \delta 21.1,55.1,112.1,116.4,119.7,120.7,123.3,124.4,125.8$, $127.8,129.1,129.3,130.0,133.8,137.2,137.8,139.4,144.0$; IR (neat) $\vee 1327,1156$, 1089, 1011, $747 \mathrm{~cm}^{-1}$; HRMS: calcd for $\left(\mathrm{M}+\mathrm{NH}_{4}{ }^{+}\right) \quad \mathrm{C}_{22} \mathrm{H}_{23} \mathrm{~N}_{3} \mathrm{O}_{2} \mathrm{SCl}$ : 428.1200; found 428.1194.

$(+)-3$ Ac This product was obtained as a colorless solid in $98 \%$ yield and $93 \%$ ee from a reaction catalyzed by $6(10 \mathrm{~mol} \%)$ at $50{ }^{\circ} \mathrm{C}$ for $28 \mathrm{~h}$.

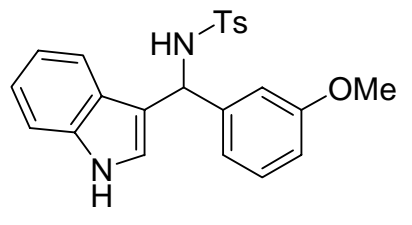

(-)-3Ad This product was obtained as a pink solid in 94\% yield after flash chromatography (silica gel: Ethyl acetate / Hexanes = $1 / 2$ ) and in $92 \%$ ee as determined by HPLC [Daicel Chiralcel $\mathrm{OD}$, Hexanes $/ \mathrm{IPA}=60 / 40,1.0 \mathrm{ml} \cdot \mathrm{min}^{-1}, \lambda=220 \mathrm{~nm}, \mathrm{t}($ major $)=11.7 \mathrm{~min}, \mathrm{t}($ minor $)=$ $8.5 \mathrm{~min}]$ from a reaction catalyzed by $7(10 \mathrm{~mol} \%)$ at $50{ }^{\circ} \mathrm{C}$ for $72 \mathrm{~h} ;[\alpha]_{\mathrm{D}}{ }^{25}=-13.2(\mathrm{c}$ 1.0, Acetone); m. p. $165-166{ }^{\circ} \mathrm{C} ;{ }^{1} \mathrm{H}$ NMR $\left(400 \mathrm{MHz}, \mathrm{CDCl}_{3}\right) \delta 2.35$ (s, 3H), 3.66 (s, $3 \mathrm{H}), 5.22(\mathrm{~d}, J=6.8 \mathrm{~Hz}, 1 \mathrm{H}), 5.81(\mathrm{~d}, J=6.8 \mathrm{~Hz}, 1 \mathrm{H}), 6.65(\mathrm{~s}, 1 \mathrm{H}), 6.68-6.75(\mathrm{~m}, 2 \mathrm{H})$, $6.80(\mathrm{~d}, J=7.6 \mathrm{~Hz}, 1 \mathrm{H}), 6.99(\mathrm{t}, J=7.2 \mathrm{~Hz}, 1 \mathrm{H}), 7.02-7.18(\mathrm{~m}, 4 \mathrm{H}), 7.20-7.32(\mathrm{~m}, 2 \mathrm{H})$, $7.53(\mathrm{~d}, J=7.6 \mathrm{~Hz}, 2 \mathrm{H}), 8.02(\mathrm{br}, 1 \mathrm{H}) ;{ }^{13} \mathrm{C} \mathrm{NMR}\left(100 \mathrm{MHz}, \mathrm{CDCl}_{3}\right) \delta 21.4,55.0,55.1$, 111.3, 112.6, 113.0, 116.1, 119.2, 119.6, 119.9, 122.4, 123.8, 125.3, 127.1, 129.2, 129.3, 136.5, 137.4, 141.8, 143.0, 159.4; IR (neat) v 1457, 1320, 1156, 1092, 1035, $745 \mathrm{~cm}^{-1}$; HRMS: calcd for $\left(\mathrm{M}+\mathrm{NH}_{4}{ }^{+}\right) \mathrm{C}_{23} \mathrm{H}_{26} \mathrm{~N}_{3} \mathrm{O}_{3} \mathrm{~S}: 424.1695$; found 424.1687 .

(+)-3Ad This product was obtained as a pink solid in $95 \%$ yield and $90 \%$ ee from a reaction catalyzed by $6(10 \mathrm{~mol} \%)$ at $50{ }^{\circ} \mathrm{C}$ for $72 \mathrm{~h}$.

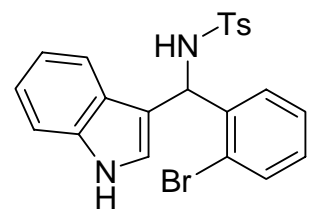

(-)-3Ae This product was obtained as a colorless solid in $98 \%$ yield after flash chromatography (silica gel: Ethyl acetate / Hexanes $=1 / 2$ ) and in $93 \%$ ee as determined by HPLC [Daicel Chiralcel OD, Hexanes $/ \mathrm{IPA}=60 / 40,1.0 \mathrm{ml} \cdot \mathrm{min}^{-1}, \lambda=220 \mathrm{~nm}, \mathrm{t}($ major $)=11.3 \mathrm{~min}, \mathrm{t}($ minor $)=5.6$ $\mathrm{min}]$ from a reaction catalyzed by $7(10 \mathrm{~mol} \%)$ at $50{ }^{\circ} \mathrm{C}$ for $18 \mathrm{~h} ;[\alpha]_{\mathrm{D}}{ }^{25}=-67.8(c=1.0$, Acetone); m. p. $201-202{ }^{\circ} \mathrm{C} ;{ }^{1} \mathrm{H}$ NMR $\left(400 \mathrm{MHz}, \mathrm{CD}_{3} \mathrm{COCD}_{3}\right) \delta 2.35$ (s, 3H), 6.30 (d, $J$ 
$=8.0 \mathrm{~Hz}, 1 \mathrm{H}), 6.55(\mathrm{~s}, 1 \mathrm{H}), 6.94(\mathrm{t}, J=7.6 \mathrm{~Hz}, 1 \mathrm{H}), 7.10(\mathrm{q}, J=8.4 \mathrm{~Hz}, 2 \mathrm{H}), 7.14-7.26$ (m, 4H), 7.33 (t, $J=9.2 \mathrm{~Hz}, 2 \mathrm{H}), 7.46(\mathrm{~d}, J=8.0 \mathrm{~Hz}, 1 \mathrm{H}), 7.60-7.70(\mathrm{~m}, 3 \mathrm{H}), 10.09$ (br, $1 \mathrm{H}) ;{ }^{13} \mathrm{C}$ NMR $\left(100 \mathrm{MHz}, \mathrm{CD}_{3} \mathrm{COCD}_{3}\right) \delta 20.7,54.0,111.6,114.9,119.1,119.3,122.0$, 123.0, 124.7, 126.4, 127.2, 127.6, 128.7, 129.3, 129.4, 132.6, 137.1, 138.9, 140.9, 142.9; IR (neat) $v$ 1440, 1328, 1158, 1020, $744 \mathrm{~cm}^{-1}$; HRMS: calcd for $\left(\mathrm{M}+\mathrm{NH}_{4}{ }^{+}\right)$ $\mathrm{C}_{22} \mathrm{H}_{23} \mathrm{~N}_{3} \mathrm{O}_{2} \mathrm{SBr}$ : 472.0694; found 472.0692 .

$(+)-3$ Ae This product was obtained as a colorless solid in $98 \%$ yield and $93 \%$ ee from a reaction catalyzed by $6(10 \mathrm{~mol} \%)$ at $50{ }^{\circ} \mathrm{C}$ for $18 \mathrm{~h}$.

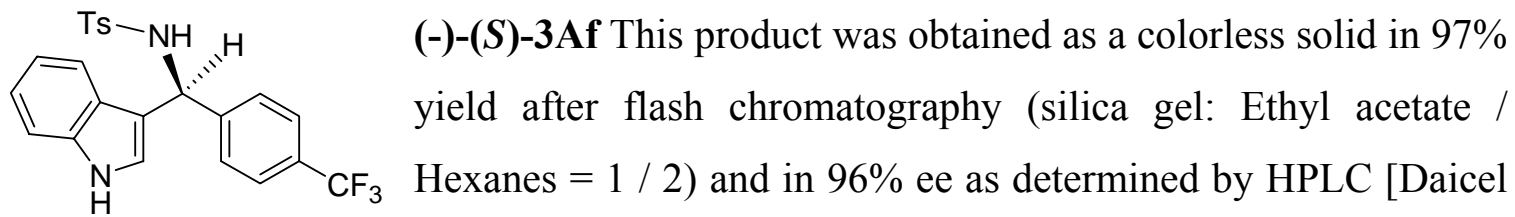
Chiralcel OD, Hexanes $/ \mathrm{IPA}=60 / 40,1.0 \mathrm{ml} \cdot \mathrm{min}^{-1}, \lambda=220 \mathrm{~nm}, \mathrm{t}$ (major) $=11.9 \mathrm{~min}, \mathrm{t}$ $($ minor $)=7.4 \mathrm{~min}]$ from a reaction catalyzed by $7(10 \mathrm{~mol} \%)$ at $50{ }^{\circ} \mathrm{C}$ for $8 \mathrm{~h}$; The absolute configuration of (-)-3Af was determined as $\boldsymbol{S}$ by X-ray diffraction as detailed below (the sample for X-ray analysis was obtained by recrystallization from acetone / hexane). $[\alpha]_{\mathrm{D}}{ }^{25}=-34.3$ (c 1.0, Acetone); m. p. 213-214 ${ }^{\circ} \mathrm{C} ;{ }^{1} \mathrm{H}$ NMR $(400 \mathrm{MHz}$, $\left.\mathrm{CD}_{3} \mathrm{COCD}_{3}\right) \delta 2.31(\mathrm{~s}, 3 \mathrm{H}), 5.96(\mathrm{~d}, J=8.0 \mathrm{~Hz}, 1 \mathrm{H}), 6.77(\mathrm{~s}, 1 \mathrm{H}), 6.93(\mathrm{t}, J=7.6 \mathrm{~Hz}$, 1H), 7.08 (t, $J=8.0 \mathrm{~Hz}, 1 \mathrm{H}), 7.13(\mathrm{~d}, J=8.4 \mathrm{~Hz}, 2 \mathrm{H}), 7.25$ (d, $J=7.6 \mathrm{~Hz}, 1 \mathrm{H}), 7.28-7.38$ (m, 2H), 7.44-7.60 (m, 6H), $10.14(\mathrm{br}, 1 \mathrm{H}) ;{ }^{13} \mathrm{C}$ NMR (100 MHz, $\left.\mathrm{CD}_{3} \mathrm{COCD}_{3}\right) \delta 20.6$, $54.8,111.6,115.4,119.3,122.0,124.3,125.0,125.1,126.0,127.2,128.3,129.3,137.2$, 139.0, 142.7, 146.3; IR (neat) $v 1423,1326,1158,1116,753 \mathrm{~cm}^{-1}$; HRMS: calcd for $\left(\mathrm{M}+\mathrm{NH}_{4}{ }^{+}\right) \mathrm{C}_{23} \mathrm{H}_{23} \mathrm{~N}_{3} \mathrm{O}_{2} \mathrm{SF}_{3}: 462.1463$; found 462.1483 .

$(+)-(\boldsymbol{R})$-3Af This product was obtained as a colorless solid in 97\% yield and $97 \%$ ee from a reaction catalyzed by $6(10 \mathrm{~mol} \%)$ at $50{ }^{\circ} \mathrm{C}$ for $8 \mathrm{~h}$.

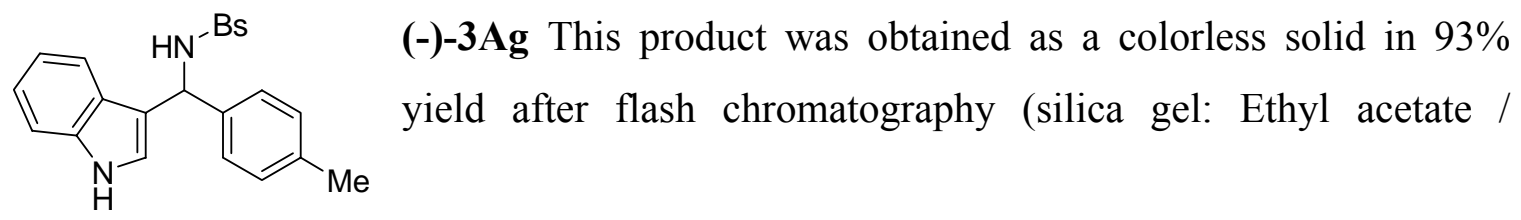


Hexanes $=1 / 2$ ) and in 92\% ee as determined by HPLC [Daicel Chiralcel OD, Hexanes / $\mathrm{IPA}=60 / 40,1.0 \mathrm{ml} \cdot \mathrm{min}^{-1}, \lambda=220 \mathrm{~nm}, \mathrm{t}$ (major) $=10.5 \mathrm{~min}, \mathrm{t}($ minor $\left.)=6.8 \mathrm{~min}\right]$ from a reaction catalyzed by $7(10 \mathrm{~mol} \%)$ at $50{ }^{\circ} \mathrm{C}$ for $46 \mathrm{~h}$; m. p. $147-148{ }^{\circ} \mathrm{C} ;{ }^{1} \mathrm{H}$ NMR $(400$ $\left.\mathrm{MHz}, \mathrm{CDCl}_{3}\right) \delta 2.27(\mathrm{~s}, 3 \mathrm{H}), 5.21(\mathrm{~d}, J=7.2 \mathrm{~Hz}, 1 \mathrm{H}), 5.82(\mathrm{~d}, J=7.2 \mathrm{~Hz}, 1 \mathrm{H}), 6.65$ (s, 1H), 6.94-7.02 (m, $3 \mathrm{H}), 7.08$ (d, $J=7.6 \mathrm{~Hz}, 2 \mathrm{H}), 7.14$ (t, $J=7.2 \mathrm{~Hz}, 1 \mathrm{H}), 7.20-7.32$ (m, $4 \mathrm{H}), 7.43$ (t, $J=7.2 \mathrm{~Hz}, 1 \mathrm{H}), 7.65(\mathrm{~d}, J=8.4 \mathrm{~Hz}, 2 \mathrm{H}), 7.97(\mathrm{br}, 1 \mathrm{H}) ;{ }^{13} \mathrm{C}$ NMR $(100$ $\left.\mathrm{MHz}, \mathrm{CDCl}_{3}\right) \delta 21.7,55.6,112.0,117.0,119.9,120.6,123.1,124.4,126.0,127.8,129.3$, 129.7, 132.8, 137.2, 137.7, 137.8, 141.0; IR (neat) $\vee 1447,1319,1158,1029,744 \mathrm{~cm}^{-1}$; HRMS: calcd for $\left(\mathrm{M}^{+}\right) \mathrm{C}_{22} \mathrm{H}_{20} \mathrm{~N}_{2} \mathrm{O}_{2} \mathrm{~S}$ : 376.1246; found 376.1239.

$(+)-3$ Ag This product was obtained as a colorless solid in $96 \%$ yield and $94 \%$ ee from a reaction catalyzed by $6(10 \mathrm{~mol} \%)$ at $50{ }^{\circ} \mathrm{C}$ for $46 \mathrm{~h} .[\alpha]_{\mathrm{D}}{ }^{25}=+12.6\left(c 1.0, \mathrm{CHCl}_{3}\right)$.

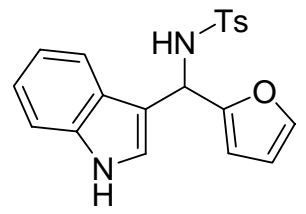

$(+)-3 \mathbf{A h}$ This product was obtained as a pink solid in $88 \%$ yield after flash chromatography (silica gel: Ethyl acetate / Hexanes $=1 / 2$ ) and in $96 \%$ ee as determined by HPLC [Daicel Chiralcel OD, Hexanes / $\mathrm{IPA}=60 / 40,1.0 \mathrm{ml} \cdot \mathrm{min}^{-1}, \lambda=220 \mathrm{~nm}, \mathrm{t}$ (major) $=11.7 \mathrm{~min}, \mathrm{t}($ minor $\left.)=6.9 \mathrm{~min}\right]$ from a reaction catalyzed by $7(10 \mathrm{~mol} \%)$ at $50{ }^{\circ} \mathrm{C}$ for $12 \mathrm{~h}$; ); m. p. $50-53{ }^{\circ} \mathrm{C} ;{ }^{1} \mathrm{H}$ NMR $(400$ $\left.\mathrm{MHz}, \mathrm{CDCl}_{3}\right) \delta 2.34(\mathrm{~s}, 3 \mathrm{H}), 5.19$ (d, $\left.J=7.6 \mathrm{~Hz}, 1 \mathrm{H}\right), 5.90$ (d, $\left.J=7.6 \mathrm{~Hz}, 1 \mathrm{H}\right), 6.07$ (d, $J$ $=2.8 \mathrm{~Hz}, 1 \mathrm{H}), 6.14-6.18(\mathrm{~m}, 1 \mathrm{H}), 6.89(\mathrm{~d}, J=2.4 \mathrm{~Hz}, 1 \mathrm{H}), 7.02(\mathrm{~d}, J=8.0 \mathrm{~Hz}, 1 \mathrm{H}), 7.09$ (d, $J=8.0 \mathrm{~Hz}, 2 \mathrm{H}), 7.15$ (t, $J=7.6 \mathrm{~Hz}, 1 \mathrm{H}), 7.19(\mathrm{~s}, 1 \mathrm{H}), 7.27$ (d, $J=8.4 \mathrm{~Hz}, 1 \mathrm{H}), 7.39$ (d, $J=8.0 \mathrm{~Hz}, 1 \mathrm{H}), 7.56(\mathrm{~d}, J=7.6 \mathrm{~Hz}, 2 \mathrm{H}), 8.08(\mathrm{br}, 1 \mathrm{H}) ;{ }^{13} \mathrm{C} \mathrm{NMR}\left(100 \mathrm{MHz}, \mathrm{CDCl}_{3}\right)$ $\delta$ 22.1, 49.8, 108.4, 110.8, 112.0, 114.2, 119.9, 120.6, 123.1, 124.1, 125.9, 127.7, 129.9, 137.0, 138.0, 142.7, 143.6, 153.2; IR (neat) v 1423, 1324, 1158, 1011, $742 \mathrm{~cm}^{-1}$; HRMS: calcd for $\left(\mathrm{M}+\mathrm{NH}_{4}{ }^{+}\right) \quad \mathrm{C}_{20} \mathrm{H}_{22} \mathrm{~N}_{3} \mathrm{O}_{3} \mathrm{~S}: 384.1382$; found 384.1372 .

(-)-3Ah This product was obtained as a pink solid in $89 \%$ yield and $94 \%$ ee from a reaction catalyzed by $6(10 \mathrm{~mol} \%)$ at $50{ }^{\circ} \mathrm{C}$ for $12 \mathrm{~h} ;[\alpha]_{\mathrm{D}}^{25}=-6.9\left(c 0.8, \mathrm{CHCl}_{3}\right)$. 


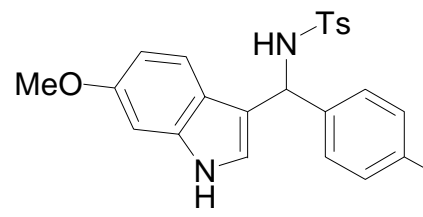

(-)-3Df This product was obtained as a white solid (132 $\mathrm{mg}$ ) in $92 \%$ yield after flash chromatography (silica gel $\mathrm{CF}_{3}$ Ethyl acetate / Hexanes $=1 / 3$ ) and in $95 \%$ ee as determined by HPLC [Daicel Chiralcel OD, Hexanes / IPA $=70 / 30,1.0 \mathrm{ml} \cdot \mathrm{min}^{-1}, \lambda=$ $254 \mathrm{~nm}, \mathrm{t}($ major $)=16.4 \mathrm{~min}, \mathrm{t}(\operatorname{minor})=8.7 \mathrm{~min}]$ from a reaction catalyzed by $7(10$ mol\%) in ethyl acetate $(400 \mu \mathrm{L})$ at $50{ }^{\circ} \mathrm{C}$ for $36 \mathrm{~h} .[\alpha]_{\mathrm{D}}{ }^{25}=-19.8(c 1.0$, Acetone); m. p. 214-216 ${ }^{\circ} \mathrm{C} ;{ }^{1} \mathrm{H}$ NMR (400 MHz, $\left.\mathrm{CD}_{3} \mathrm{COCD}_{3}\right) \delta 2.32(\mathrm{~s}, 3 \mathrm{H}), 3.76$ (s, 3H), $5.94(\mathrm{~d}, J=$ $6.4 \mathrm{~Hz}, 1 \mathrm{H}), 6.63(\mathrm{~d}, J=2.0 \mathrm{~Hz}, 1 \mathrm{H}), 6.65(\mathrm{~s}, 1 \mathrm{H}), 6.92(\mathrm{~d}, J=2.0 \mathrm{~Hz}, 1 \mathrm{H}), 7.13(\mathrm{~d}, J=$ $8.0 \mathrm{~Hz}, 2 \mathrm{H}), 7.25(\mathrm{~d}, J=8.0 \mathrm{~Hz}, 2 \mathrm{H}), 7.44-7.62(\mathrm{~m}, 6 \mathrm{H}), 9.98(\mathrm{br}, 1 \mathrm{H}) ;{ }^{13} \mathrm{C}$ NMR $(100$ $\left.\mathrm{MHz}, \mathrm{CD}_{3} \mathrm{COCD}_{3}\right) \delta$ 20.6, 55.0, 94.58, 94.62, 109.6, 119.9, 122.9, 123.0, 124.97, 125.00, $127.2,128.3,128.5,137.9,138.0,138.89,138.93,142.7,146.2,156.8$; IR (neat) v 3421, 3311, 1324, 1158; HRMS: calcd for $\left(\mathrm{M}+\mathrm{Na}^{+}\right) \mathrm{C}_{24} \mathrm{H}_{21} \mathrm{~N}_{2} \mathrm{O}_{3} \mathrm{NaSF}_{3}$ : 497.1123; found 497.1111.

(+)-3Df This product was obtained as a white solid (144 mg) in 99\% yield and 94\% ee from a reaction catalyzed by $6(10 \mathrm{~mol} \%)$ ethyl acetate $(400 \mu \mathrm{l})$ at $50{ }^{\circ} \mathrm{C}$ for $36 \mathrm{~h}$.

(-)-3Cd This product was obtained as a white solid (126
$\mathrm{mg})$ in $86 \%$ yield after flash chromatography (silica gel,
Ethyl acetate / Hexanes $=1 / 3$ ) and in $86 \%$ ee as determined by HPLC [Daicel Chiralcel OD, Hexanes / IPA $=70 / 30,1.0 \mathrm{ml} \cdot \mathrm{min}^{-1}, \lambda=$ $254 \mathrm{~nm}, \mathrm{t}($ major $)=10.3 \mathrm{~min}, \mathrm{t}($ minor $)=8.0 \mathrm{~min}]$ from a reaction catalyzed by $7(10$ mol\%) at $70{ }^{\circ} \mathrm{C}$ for $72 \mathrm{~h}$; m. p. $179-180{ }^{\circ} \mathrm{C} ;{ }^{1} \mathrm{H}$ NMR (400 MHz, $\left.\mathrm{CD}_{3} \mathrm{COCD}_{3}\right) \delta 2.32$ (s, $3 \mathrm{H}), 3.68(\mathrm{~s}, 3 \mathrm{H}), 5.85(\mathrm{~d}, J=8.4 \mathrm{~Hz}, 1 \mathrm{H}), 6.73(\mathrm{dd}, J=2.0,8.8 \mathrm{~Hz}, 1 \mathrm{H}), 6.84-6.94(\mathrm{~m}$, $3 \mathrm{H}), 7.02-7.22(\mathrm{~m}, 5 \mathrm{H}) .7 .35(\mathrm{t}, J=8.8 \mathrm{~Hz}, 1 \mathrm{H}), 7.68(\mathrm{~d}, J=8.0 \mathrm{~Hz}, 3 \mathrm{H}), 10.23(\mathrm{br}, 1 \mathrm{H})$; ${ }^{13} \mathrm{C}$ NMR $\left(100 \mathrm{MHz}, \mathrm{CD}_{3} \mathrm{COCD}_{3}\right) \delta 20.7,54.7,55.0,112.8,113.0,114.4,114.9,116.6$, 119.8, 121.1, 122.1, 125.2, 125.3, 127.1, 129.1, 129.2, 138.0, 139.2, 142.5, 142.9, 159.9; IR (neat) v 3355, 1157; HRMS: calcd for $\left(\mathrm{M}+\mathrm{Na}^{+}\right) \mathrm{C}_{23} \mathrm{H}_{21} \mathrm{~N}_{2} \mathrm{O}_{3} \mathrm{BrNa}$ : 507.0354; found 507.0346 . 
$(+)-3 C d$ This product was obtained as a white solid $(122 \mathrm{mg})$ in $83 \%$ yield and $83 \%$ ee from a reaction catalyzed by $6(10 \mathrm{~mol} \%)$ at $70{ }^{\circ} \mathrm{C}$ for $72 \mathrm{~h} .[\alpha]_{\mathrm{D}}{ }^{25}=+18.4(c 0.9$, Acetone);

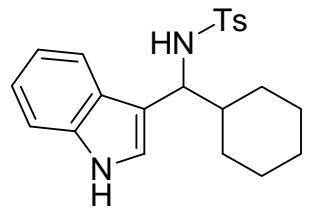

(-)-3Ai This product was obtained as a colorless solid in $86 \%$ yield after flash chromatography (silica gel: Ethyl acetate / Hexanes $=1 / 2$ ) and in $94 \%$ ee as determined by HPLC [Daicel Chiralcel OD, Hexanes $/ \mathrm{IPA}=60 / 40,1.0 \mathrm{ml} \cdot \mathrm{min}^{-1}, \lambda=220 \mathrm{~nm}, \mathrm{t}$ (major) $=5.8 \mathrm{~min}, \mathrm{t}($ minor $)=4.5$ $\mathrm{min}]$ from a reaction catalyzed by $7(10 \mathrm{~mol} \%)$ at $50{ }^{\circ} \mathrm{C}$ for $48 \mathrm{~h} ;[\alpha]_{\mathrm{D}}{ }^{25}=-25.4(c 1.0$, $\mathrm{CHCl}_{3}$ ); m. p. $80-82{ }^{\circ} \mathrm{C} ;{ }^{1} \mathrm{H}$ NMR $\left(400 \mathrm{MHz}, \mathrm{CDCl}_{3}\right) \delta$ 0.82-1.30 (m, 5H), 1.39-.49 (m, 1H), 1.51-1.64 (m, 2H), 1.66-1.77 (m, 1H), 1.78-1.90 (m, $1 \mathrm{H}), 1.98-2.09(\mathrm{~m}, 1 \mathrm{H}), 2.21$ (s, 3H), 4.35 (t, $J=8.0 \mathrm{~Hz}, 1 \mathrm{H}), 5.30$ (d, $J=8.0 \mathrm{~Hz}, 1 \mathrm{H}), 6.73$ (s, 1H), 6.87 (d, $J=8.0$ Hz, 2H), 6.98 (t, $J=7.6 \mathrm{~Hz}, 1 \mathrm{H}), 7.09$ (t, $J=7.6 \mathrm{~Hz}, 1 \mathrm{H}), 7.20(\mathrm{~d}, J=8.0 \mathrm{~Hz}, 1 \mathrm{H}), 7.35-$ $7.44(\mathrm{~m}, 3 \mathrm{H}), 7.93(\mathrm{br}, 1 \mathrm{H}) ;{ }^{13} \mathrm{C}$ NMR $\left(100 \mathrm{MHz}, \mathrm{CDCl}_{3}\right) \delta 22.0,26.6,26.9,30.5,30.6$, 43.6, 57.8, 111.8, 115.6, 119.8, 120.1, 122.5, 123.2, 126.1, 127.5, 129.3, 136.8, 138.0, 143.0; IR (neat) $\vee$ 2925, 1429, 1318, 1156, $740 \mathrm{~cm}^{-1}$; HRMS: calcd for $\left(\mathrm{M}+\mathrm{NH}_{4}{ }^{+}\right)$ $\mathrm{C}_{22} \mathrm{H}_{30} \mathrm{~N}_{3} \mathrm{O}_{2} \mathrm{~S}: 400.2059$; found 400.2063 .

(+)-3Ai This product was obtained as a colorless solid in $88 \%$ yield and $92 \%$ ee from a reaction catalyzed by $6(10 \mathrm{~mol} \%)$ at $50{ }^{\circ} \mathrm{C}$ for $48 \mathrm{~h}$.

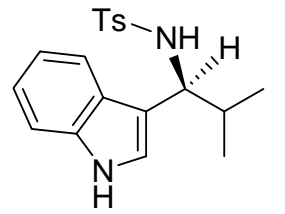

(-)-(S)-3Aj This product was obtained as a colorless gum in $85 \%$ yield after flash chromatography (silica gel: Ethyl acetate / Hexanes $=1 / 2$ ) and in $94 \%$ ee as determined by HPLC [Daicel Chiralcel OD, Hexanes / $\mathrm{IPA}=60 / 40,1.0 \mathrm{ml} \cdot \mathrm{min}^{-1}, \lambda=220 \mathrm{~nm}, \mathrm{t}$ (major) $=5.4 \mathrm{~min}, \mathrm{t}($ minor $\left.)=4.6 \mathrm{~min}\right]$ from a reaction catalyzed by $7(10 \mathrm{~mol} \%)$ at $50{ }^{\circ} \mathrm{C}$ for $48 \mathrm{~h} ;{ }^{1} \mathrm{H}$ NMR $\left(400 \mathrm{MHz}, \mathrm{CDCl}_{3}\right) \delta 0.79$ (d, $J=6.8 \mathrm{~Hz}, 3 \mathrm{H}), 0.97$ (d, $J=6.8 \mathrm{~Hz}, 3 \mathrm{H}), 2.21(\mathrm{~m}, 4 \mathrm{H}), 4.32(\mathrm{t}, J=8.0 \mathrm{~Hz}, 1 \mathrm{H}), 5.04$ (d, $J=8.0 \mathrm{~Hz}, 1 \mathrm{H}), 6.75(\mathrm{~d}, J=2.4 \mathrm{~Hz}, 1 \mathrm{H}), 6.88(\mathrm{~d}, J=8.0 \mathrm{~Hz}, 2 \mathrm{H}), 6.97(\mathrm{t}, J=7.6 \mathrm{~Hz}$, $1 \mathrm{H}), 7.08(\mathrm{t}, J=8.0 \mathrm{~Hz}, 1 \mathrm{H}), 7.19(\mathrm{~d}, J=8.4 \mathrm{~Hz}, 1 \mathrm{H}), 7.38(\mathrm{~d}, J=8.0 \mathrm{~Hz}, 1 \mathrm{H}), 7.42(\mathrm{~d}, J$ $=8.8 \mathrm{~Hz}, 2 \mathrm{H}), 7.90(\mathrm{br}, 1 \mathrm{H}) ;{ }^{13} \mathrm{C} \mathrm{NMR}\left(100 \mathrm{MHz}, \mathrm{CDCl}_{3}\right) \delta 19.9,20.1,22.0,34.1,58.6$, 
$111.8,115.8,119.7,120.1,122.6,123.1,126.1,127.5,129.4,136.8,138.0,143.1$; IR (neat) $\vee$ 2956, 1458, 1323, 1158, 1093, $742 \mathrm{~cm}^{-1}$; HRMS: calcd for $\left(\mathrm{M}+\mathrm{NH}_{4}{ }^{+}\right)$ $\mathrm{C}_{19} \mathrm{H}_{26} \mathrm{~N}_{3} \mathrm{O}_{2} \mathrm{~S}$ : 360.1746; found 360.1767 .

$(+)-(R)-3 A \mathbf{j}$ This product was obtained as a colorless gum in $84 \%$ yield and $94 \%$ ee from a reaction catalyzed by $6(10 \mathrm{~mol} \%)$ at $50{ }^{\circ} \mathrm{C}$ for $48 \mathrm{~h} ;[\alpha]_{\mathrm{D}}{ }^{25}=+36.6\left(c 1.0, \mathrm{CHCl}_{3}\right)$; The absolute configuration of (+)-3Af was determined as $\boldsymbol{R}$ by the modified Mosher's method as described in detail below.

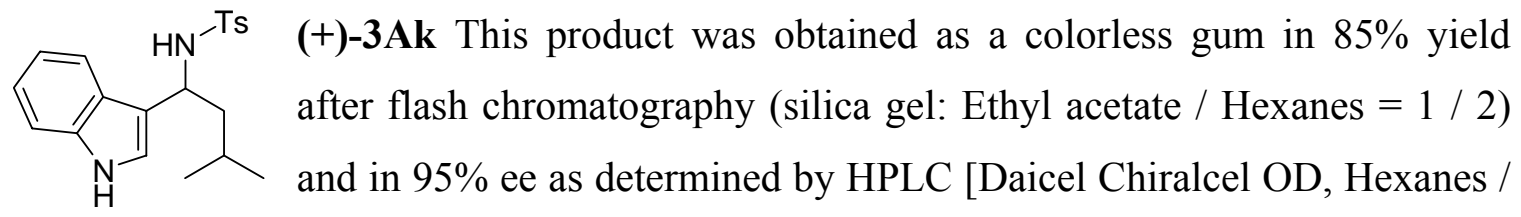
$\mathrm{IPA}=60 / 40,1.0 \mathrm{ml} \cdot \mathrm{min}^{-1}, \lambda=220 \mathrm{~nm}, \mathrm{t}$ (major) $=11.4 \mathrm{~min}, \mathrm{t}($ minor $\left.)=7.4 \mathrm{~min}\right]$ from a reaction catalyzed by $7(10 \mathrm{~mol} \%)$ at $50{ }^{\circ} \mathrm{C}$ for $12 \mathrm{~h} ;{ }^{1} \mathrm{H}$ NMR $\left(400 \mathrm{MHz}, \mathrm{CDCl}_{3}\right) \delta 0.78$ - $0.92(\mathrm{~m}, 6 \mathrm{H}), 1.44-1.57(\mathrm{~m}, 1 \mathrm{H}), 1.76-1.95(\mathrm{~m}, 2 \mathrm{H}), 2.31(\mathrm{~s}, 3 \mathrm{H}), 4.59-4.74(\mathrm{~m}, 1 \mathrm{H})$, 4.75-4.78 (m, $1 \mathrm{H}), 6.88(\mathrm{~s}, 1 \mathrm{H}), 6.94-7.05(\mathrm{~m}, 3 \mathrm{H}), 7.13(\mathrm{t}, J=7.6 \mathrm{~Hz}, 1 \mathrm{H}), 7.25(\mathrm{~d}, J=$ $9.2 \mathrm{~Hz}, 1 \mathrm{H}), 7.30(\mathrm{~d}, J=7.6 \mathrm{~Hz}, 1 \mathrm{H}), 7.51(\mathrm{~d}, J=8.0 \mathrm{~Hz}, 2 \mathrm{H}), 7.92(\mathrm{br}, 1 \mathrm{H}) ;{ }^{13} \mathrm{C} \mathrm{NMR}$ $\left(100 \mathrm{MHz}, \mathrm{CDCl}_{3}\right) \delta 22.1,22.7,23.4,25.7,46.1,50.8,111.9,116.5,119.7,120.3,122.8$, 122.9, 125.8, 127.7, 129.6 , 137.0, 138.2, 143.3; IR (neat) v 2957, 1457, 1320, 1158, 742 $\mathrm{cm}^{-1}$; HRMS: calcd for $\left(\mathrm{M}+\mathrm{NH}_{4}{ }^{+}\right) \mathrm{C}_{20} \mathrm{H}_{28} \mathrm{~N}_{3} \mathrm{O}_{2} \mathrm{~S}$ : 374.1902; found 374.1895.

$(+)-3 A k$ This product was obtained as a colorless gum in $85 \%$ yield and $95 \%$ ee from a reaction catalyzed by $6(10 \mathrm{~mol} \%)$ at $50{ }^{\circ} \mathrm{C}$ for $12 \mathrm{~h} ;[\alpha]_{\mathrm{D}}{ }^{25}=+29.7\left(c 1.0, \mathrm{CHCl}_{3}\right)$.

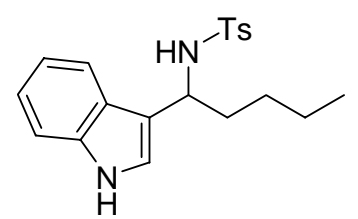

(-)-3Al This product was obtained as a colorless gum in 53\% yield after flash chromatography (silica gel: Ethyl acetate / Hexanes $=1$ / 2) and in $96 \%$ ee as determined by HPLC [Daicel Chiralcel OD, Hexanes $/ \mathrm{IPA}=60 / 40,1.0 \mathrm{ml} \cdot \mathrm{min}^{-1}, \lambda=220 \mathrm{~nm}, \mathrm{t}$ (major) $=12.4 \mathrm{~min}, \mathrm{t}$ (minor) $=8.3$ min] from a reaction catalyzed by $7(10 \mathrm{~mol} \%)$ at $50{ }^{\circ} \mathrm{C}$ for $12 \mathrm{~h} ;{ }^{1} \mathrm{H} \mathrm{NMR}(400 \mathrm{MHz}$, $\left.\mathrm{CDCl}_{3}\right) \delta$ 0.70-0.90 (m, 3H), 1.05-1.35 (m, 4H), 1.90-2.02 (m, 2H), $2.32(\mathrm{~s}, 3 \mathrm{H}), 4.50-$ 
$4.60(\mathrm{~m}, 1 \mathrm{H}), 4.80-4.87(\mathrm{~m}, 1 \mathrm{H}), 6.88(\mathrm{~d}, J=2.4 \mathrm{~Hz}, 1 \mathrm{H}), 6.98(\mathrm{t}, J=8.0 \mathrm{~Hz}, 1 \mathrm{H}), 7.05$ $(\mathrm{d}, J=8.0 \mathrm{~Hz}, 2 \mathrm{H}), 7.13$ (t, $J=8.0 \mathrm{~Hz}, 1 \mathrm{H}), 7.26$ (t, $J=4.8 \mathrm{~Hz}, 2 \mathrm{H}), 7.32$ (d, $J=8.0 \mathrm{~Hz}$, $1 \mathrm{H}), 7.54(\mathrm{~d}, J=8.0 \mathrm{~Hz}, 2 \mathrm{H}), 7.94(\mathrm{br}, 1 \mathrm{H}) ;{ }^{13} \mathrm{C} \mathrm{NMR}\left(100 \mathrm{MHz}, \mathrm{CDCl}_{3}\right) \delta$ 14.6, 22.1, $22.9,29.0,36.7,52.5,111.9,116.4,119.7,120.3,122.8,122.9,125.8,127.7,129.7$, 137.1, 138.2, 143.4; IR (neat) $v$ 2957, 1427, 1323, 1158, $742 \mathrm{~cm}^{-1}$; HRMS: calc'd for $\left(\mathrm{M}+\mathrm{Na}^{+}\right) \mathrm{C}_{20} \mathrm{H}_{24} \mathrm{~N}_{2} \mathrm{O}_{2} \mathrm{SNa}$ : 379.1456; found 379.1455.

(+)-3Al This product was obtained as a colorless gum in 55\% yield and $97 \%$ ee from a reaction catalyzed by $6(10 \mathrm{~mol} \%)$ at $50{ }^{\circ} \mathrm{C}$ for $12 \mathrm{~h} ;[\alpha]_{\mathrm{D}}{ }^{25}=+19.5\left(c 1.0, \mathrm{CHCl}_{3}\right)$.

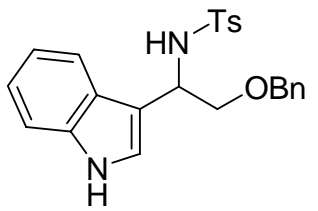

(-)-3Am This product was obtained as a colorless gum in $65 \%$ yield after flash chromatography (silica gel: Ethyl acetate / Hexanes $=1$ / 2) and in $96 \%$ ee as determined by HPLC [Daicel Chiralcel OD, Hexanes $/ \mathrm{IPA}=80 / 20,1.0 \mathrm{ml} \cdot \mathrm{min}^{-1}, \lambda=220 \mathrm{~nm}, \mathrm{t}($ major $)=21.7 \mathrm{~min}, \mathrm{t}($ minor $)=16.2$ min] from a reaction catalyzed by $7(10 \mathrm{~mol} \%)$ at $-25{ }^{\circ} \mathrm{C}$ for $\left.10 \mathrm{~h} ;\right) ;{ }^{1} \mathrm{H}$ NMR $(400 \mathrm{MHz}$, $\left.\mathrm{CDCl}_{3}\right) \delta 2.31(\mathrm{~s}, 3 \mathrm{H}), 3.68-3.74(\mathrm{~m}, 1 \mathrm{H}), 3.75-3.84(\mathrm{~m}, 1 \mathrm{H}), 4.46(\mathrm{~s}, 2 \mathrm{H}), 4.79-4.86(\mathrm{~m}$, $1 \mathrm{H}), 5.20(\mathrm{~d}, J=4.8 \mathrm{~Hz}, 1 \mathrm{H}), 6.97$ (t, $J=7.2 \mathrm{~Hz}, 1 \mathrm{H}), 7.04(\mathrm{~d}, J=8.0 \mathrm{~Hz}, 2 \mathrm{H}), 7.08(\mathrm{~s}, 1$ H), $7.12(\mathrm{t}, J=8.0 \mathrm{~Hz}, 1 \mathrm{H}), 7.18-7.38(\mathrm{~m}, 7 \mathrm{H}), 7.54$ (d, $J=8.0 \mathrm{~Hz}, 2 \mathrm{H}), 8.08$ (br, 1H); ${ }^{13} \mathrm{C}$ NMR $\left(100 \mathrm{MHz}, \mathrm{CDCl}_{3}\right) \delta 22.1,51.6,72.8,73.9,111.8,113.7,119.8,120.3,122.8$, $124.0,126.3,127.8,128.4,128.5,129.1,129.8,137.9,138.3,143.5$; IR (neat) $v 1456$, 1333, 1161, 1093, $743 \mathrm{~cm}^{-1}$; HRMS: calcd for $\left(\mathrm{M}+\mathrm{Na}^{+}\right) \quad \mathrm{C}_{24} \mathrm{H}_{24} \mathrm{~N}_{2} \mathrm{O}_{3} \mathrm{SNa}$ : 433.1405; found 433.1391 .

(+)-3Am This product was obtained as a pink solid in $65 \%$ yield and $96 \%$ ee from a reaction catalyzed by $6(10 \mathrm{~mol} \%)$ at $-25^{\circ} \mathrm{C}$ for $10 \mathrm{~h} ;[\alpha]_{\mathrm{D}}{ }^{25}=+21.7\left(c 0.9, \mathrm{CHCl}_{3}\right)$. 


\section{Deprotection of Ts Group: ${ }^{6}$}

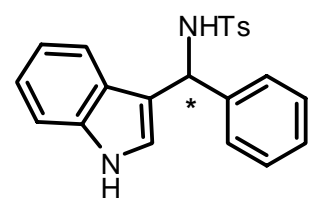

(+)-3Ab 94\% ee

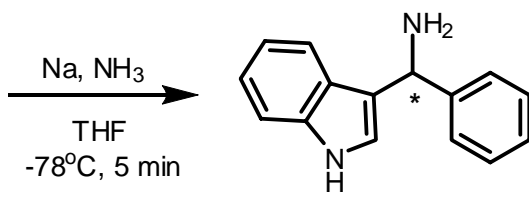

$-78^{\circ} \mathrm{C}, 5 \mathrm{~min}$

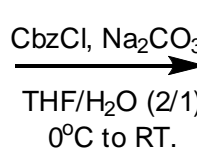

$0^{\circ} \mathrm{C}$ to RT.

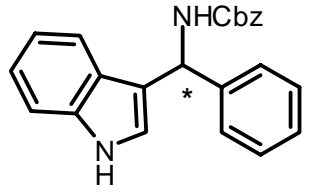

$(+)-8 \mathrm{Ab}$

98\% (2 steps) $94 \%$ ee

An oven-dried three-necked flask was charged with a solution of compound (+)-3Ab (95 $\mathrm{mg}, 0.252 \mathrm{mmol})$ in anhydrous THF $(1.0 \mathrm{~mL})$ at room temperature. To this solution at $-78^{\circ} \mathrm{C}$ was added liquid ammonia $(\sim 5 \mathrm{~mL})$, and then sodium (excess, $\sim 50 \mathrm{mg}$ ) was added in portions until a deep blue coloration persisted for $5 \mathrm{~min}$. The reaction was quenched at $-78^{\circ} \mathrm{C}$ with slow addition of solid $\mathrm{NH}_{4} \mathrm{Cl}(\sim 0.5 \mathrm{~g})$. The resulting white mixture was slowly warmed up to room temperature, at which ammonia was evaporated. The residue was partitioned between water $(5 \mathrm{~mL})$ and $\mathrm{CH}_{2} \mathrm{Cl}_{2}(5 \mathrm{~mL})$. The organic layer was collected, the aqueous layer was extracted with $\mathrm{CH}_{2} \mathrm{Cl}_{2}(4 \times 5 \mathrm{~mL})$. The combined organic phase extracts were dried over $\mathrm{Na}_{2} \mathrm{SO}_{4}$. The organic solution was concentrated. The resulting white solid (the free amine) was dissolved in THF (3 $\mathrm{mL})$, after which water $(1.5 \mathrm{~mL})$ was added. The resulting mixture was vigorously stirred at $0^{\circ} \mathrm{C}$, to which was added $\mathrm{Na}_{2} \mathrm{CO}_{3}(60 \mathrm{mg}, 0.566 \mathrm{mmol}, 2.2$ equiv). The resulting suspension was stirred at $0^{\circ} \mathrm{C}$ for $5 \mathrm{~min}$, and after which benzyl chloroformate (60 $\mu \mathrm{L}, 0.42 \mathrm{mmol}, 1.7$ equiv) was introduced to this mixture via a syringe. The reaction mixture was stirred at room temperature for an additional $10 \mathrm{~min}$, and then diluted with EtOAc $(10 \mathrm{~mL})$. The organic layer was collected, washed with brine and dried over $\mathrm{Na}_{2} \mathrm{SO}_{4}$. After removal of the solvent, the residue was subjected flash chromatography $\left(\mathrm{SiO}_{2}\right.$ petroleum ether/EtOAc: $5 / 1$ to $3 / 1$ ) to give compound (+)-8Ab (90 mg, 99\% yield) as colorless crystals. The enantiomeric excess was determined by HPLC to be $94 \%$ (Chiralcel OD, Hexanes / IPA $=60 / 40,1.0 \mathrm{~mL}, 220 \mathrm{~nm}): \mathrm{t}$ (major) $=6.4 \min , \mathrm{t}($ minor $)=7.7 \min .[\alpha]_{\mathrm{D}}{ }^{23}=+40.8(c$ 1.0, $\mathrm{CHCl}_{3}$ ); m. p. $144-145{ }^{\circ} \mathrm{C}$; ${ }^{1} \mathrm{H}$ NMR (400 MHz, $\left.\mathrm{CDCl}_{3}\right) \delta 5.13$ (two doublets, $\mathrm{AB}$ system, $J=12 \mathrm{~Hz}, 2 \mathrm{H}), 5.47$ (d, $J=7.2 \mathrm{~Hz}, 1 \mathrm{H}), 6.26$ (d, $J=7.2 \mathrm{~Hz}, 1 \mathrm{H}), 6.69$ (s, 1H), 7.06 (t, $J=8 \mathrm{~Hz}, 1 \mathrm{H}), 7.18$ (t, $J=8 \mathrm{~Hz}, 1 \mathrm{H}), 7.24-7.40(\mathrm{~m}, 11 \mathrm{H}), 7.46(\mathrm{~d}, J=8 \mathrm{~Hz}, 1 \mathrm{H})$, $8.11(\mathrm{br}, 1 \mathrm{H}) .{ }^{13} \mathrm{C}$ NMR $\left(100 \mathrm{MHz}, \mathrm{CDCl}_{3}\right) \delta 52.3,66.9,111.3,115.7,117.30,119.35$, $119.9,112.5,123.3,125.7,126.8,127.3,128.1,128.4,136.4,136.6,141.5$; IR (neat) $v$ 
3466, 3055, 1719, 1498, 1265, $742 \mathrm{~cm}^{-1}$. HR-MS: cacld $\left(\mathrm{M}+\mathrm{Na}^{+}\right)$for $\mathrm{C}_{23} \mathrm{H}_{20} \mathrm{~N}_{2} \mathrm{O}_{2}$, 379.1422; found, 379.1415 .

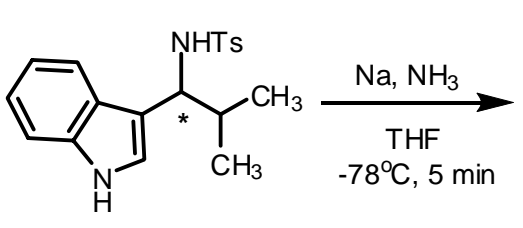

$(-)-3$ Aj $94 \%$ ee

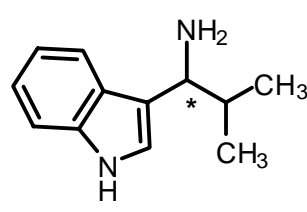
$\frac{\mathrm{CbzCl}, \mathrm{Na}_{2} \mathrm{CO}_{3}}{\stackrel{\mathrm{THF} / \mathrm{H}_{2} \mathrm{O}(2 / 1)}{0^{\circ} \mathrm{C} \text { to RT. }}}$

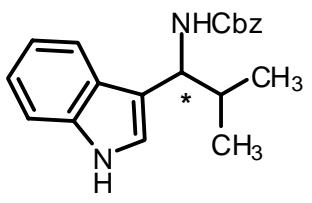

$(-)-8 \mathrm{Aj}$

97\% (2 steps), 94\%ee

An oven-dried three-necked flask was charged with a solution of compound (-)-3Aj $\mathbf{j}$ $(50 \mathrm{mg}, 0.146 \mathrm{mmol})$ in anhydrous THF $(1.0 \mathrm{~mL})$ at room temperature. To this solution at $-78^{\circ} \mathrm{C}$ was added liquid ammonia $(\sim 5 \mathrm{~mL})$, and then sodium (excess, $\sim 50 \mathrm{mg}$ ) was added in portions until a deep blue coloration persisted for $5 \mathrm{~min}$. The reaction was quenched at $-78^{\circ} \mathrm{C}$ with slow addition of solid $\mathrm{NH}_{4} \mathrm{Cl}(\sim 0.5 \mathrm{~g})$. The resulting white mixture was slowly warmed up to room temperature, at which ammonia was evaporated. The residue was partitioned between water $(5 \mathrm{~mL})$ and $\mathrm{CH}_{2} \mathrm{Cl}_{2}(5 \mathrm{~mL})$. The organic layer was collected, the aqueous layer was extracted with $\mathrm{CH}_{2} \mathrm{Cl}_{2}(4 \times 5 \mathrm{~mL})$. The combined organic phase extracts were dried over $\mathrm{Na}_{2} \mathrm{SO}_{4}$. The organic solution was concentrated and the corresponding white solid (the free amine) was dissolved in THF ( $3 \mathrm{~mL}$ ), after which water $(1.5 \mathrm{~mL})$ was added. The resulting mixture was vigorously stirred at $0^{\circ} \mathrm{C}$, to which was added $\mathrm{Na}_{2} \mathrm{CO}_{3}$ (60 mg, $0.566 \mathrm{mmol}, 3.9$ equiv.). The resulting mixture was stirred at $0^{\circ} \mathrm{C}$ for $5 \mathrm{~min}$, then benzyl chloroformate $(40 \mu \mathrm{L}, 0.28 \mathrm{mmol}, 1.9$ equiv.) was introduced to this mixture via a syringe. The reaction mixture was stirred at room temperature for an additional $10 \mathrm{~min}$, and then diluted with EtOAc $(10 \mathrm{~mL})$. The organic layer was collected, washed with brine and dried over $\mathrm{Na}_{2} \mathrm{SO}_{4}$. After removal of the solvent, the residue was subjected to flash chromatography $\left(\mathrm{SiO}_{2}\right.$ petroleum ether/EtOAc: $5 / 1$ to $3 / 1$ ) to give compound (-)-8Aj (46 $\mathrm{mg}, 97 \%$ yield) as a pale pink oil. The enantiomeric excess was determined by HPLC to be $94 \%$ (Chiralcel OD, Hexanes / IPA $=70 / 30,1.0 \mathrm{~mL}, 220 \mathrm{~nm}): \mathrm{t}($ major $)=9.8 \mathrm{~min}, \mathrm{t}($ minor $)=7.9 \mathrm{~min} .[\alpha]_{\mathrm{D}}{ }^{23}=-31.3(c$ 1.1, $\left.\mathrm{CHCl}_{3}\right) .{ }^{1} \mathrm{H}$ NMR $\left(400 \mathrm{MHz}, \mathrm{CDCl}_{3}\right) \delta \mathrm{ppm}: 0.93(\mathrm{~d}, J=6.4 \mathrm{~Hz}, 3 \mathrm{H}), 1.01(\mathrm{~d}, J=$ $6.0 \mathrm{~Hz}, 3 \mathrm{H}), 2.28(\mathrm{~m}, 1 \mathrm{H}), 4.83(\mathrm{t}, J=8.4 \mathrm{~Hz}, 1 \mathrm{H}), 5.05-5.15$ (m, 3H), 6.99 (s, 1H), 7.09 (t, $J=7.6 \mathrm{~Hz}, 1 \mathrm{H}), 7.18$ (t, $J=7.6 \mathrm{~Hz}, 1 \mathrm{H}), 7.27-7.40(\mathrm{~m}, 5 \mathrm{H}), 7.62(\mathrm{~d}, J=7.6 \mathrm{~Hz}, 1 \mathrm{H})$, 8.14 (br, $1 \mathrm{H}) ;{ }^{13} \mathrm{C}$ NMR $\left(100 \mathrm{MHz}, \mathrm{CDCl}_{3}\right) \delta \mathrm{ppm} \mathrm{18.9,} \mathrm{20.1,} \mathrm{32.8,} \mathrm{54.7,} \mathrm{66.7,} \mathrm{111.3,}$ 
116.6, 119.3, 119.6, 121.7, 122.1, 125.9, 128.0, 128.5, 136.4, 136.5, 156.2; IR (neat) $v$ $3465,3055,2964,1710,1508,1266,741 \mathrm{~cm}^{-1}$; HR-MS: cacld for $\left(\mathrm{M}+\mathrm{Na}^{+}\right) \mathrm{C}_{20} \mathrm{H}_{22} \mathrm{~N}_{2} \mathrm{O}_{2}$, 345.1579; found, 345.1574 .

\section{X-ray crystallographic anaylysis of the compound (-)-(S)-3Af obtained with 7}

Experimental Section:

A colorless plate crystal with dimensions $0.14 \times 0.10 \times 0.04 \mathrm{~mm}$ was mounted on a $0.2 \mathrm{~nm}$ nylon loop using very small amount of paratone oil.

Data were collected using a Bruker SMART CCD (charge coupled device) based diffractometer equipped with an Oxford Cryostream low-temperature apparatus operating at $193 \mathrm{~K}$. A suitable crystal was chosen and mounted on a glass fiber using grease. Data were measured using omega scans of $0.3^{\circ}$ per frame for 30 seconds, such that a hemisphere was collected. A total of 1271 frames were collected with a maximum resolution of $0.76 \AA$. The first 50 frames were recollected at the end of data collection to monitor for decay. Cell parameters were retrieved using SMART ${ }^{1}$ software and refined using SAINT on all observed reflections. Data reduction was performed using the SAINT software ${ }^{2}$ which corrects for Lp and decay. Absorption corrections were applied using SADABS ${ }^{6}$ multiscan technique, supplied by George Sheldrick. The structures are solved by the direct method using the SHELXS- $97^{3}$ program and refined by least squares method on $\mathrm{F}^{2}$, SHELXL-97, ${ }^{4}$ incorporated in SHELXTL-PC V $6.10 .{ }^{5}$

The structure was solved in the space group C2 (\# 5) All non-hydrogen atoms are refined anisotropically. Hydrogens were calculated by geometrical methods and refined as a riding model. The Flack ${ }^{7}$ parameter is used to determine chirality of the crystal studied, the value should be near zero, a value of one is the other enantimer and a value 
of 0.5 is racemic. The Flack parameter was refined to $0.03(7)$, confirming the absolute stereochemistry. The crystal used for the diffraction study showed no decomposition during data collection. All drawing are done at 50\% ellipsoids.

Acknowledgement. The CCD based x-ray diffractometer at Harvard University was purchased through NIH grant (1S10RR11937-01).

\section{References}

1. SMART V 5.625 (NT) Software for the CCD Detector System; Bruker Analytical X-ray Systems, Madison, WI (2001).

2. SAINT V 6.22 (NT) Software for the CCD Detector System Bruker Analytical Xray Systems, Madison, WI (2001).

3. Sheldrick, G. M. SHELXS-90, Program for the Solution of Crystal Structure, University of Göttingen, Germany, 1990.

4. Sheldrick, G. M. SHELXL-97, Program for the Refinement of Crystal Structure, University of Göttingen, Germany, 1997.

5. SHELXTL 6.1 (PC-Version), Program library for Structure Solution and Molecular Graphics; Bruker Analytical X-ray Systems, Madison, WI (2000).

6. SADABS. Program for absorption corrections using Siemens CCD based on the method of Robert Blessing; Blessing, R.H. Acta Cryst. A51 1995, 33-38.

7. Flack, H. D.. Acta Cryst. A39, 1983, 876-881.

${ }^{a}$ Obtained with graphite monochromated Mo K $\alpha(\lambda=0.71073 \AA)$ radiation. ${ }^{\mathrm{b}} R 1=\Sigma|| F_{\mathrm{o}} \mid-$ $\left|F_{\mathrm{c}}\right||/ \Sigma| F_{\mathrm{o}} \mid .{ }^{c} w R_{2}=\left\{\sum\left[w\left(F_{\mathrm{o}}{ }^{2}-F_{\mathrm{c}}{ }^{2}\right)^{2} /\left\{\sum\left[w\left(F_{\mathrm{o}}{ }^{2}\right)^{2}\right]\right\}^{1 / 2}\right.\right.$.

The following are $50 \%$ thermal ellipsoidal drawings of the molecule in the asymmetric cell with various amount of labeling. 

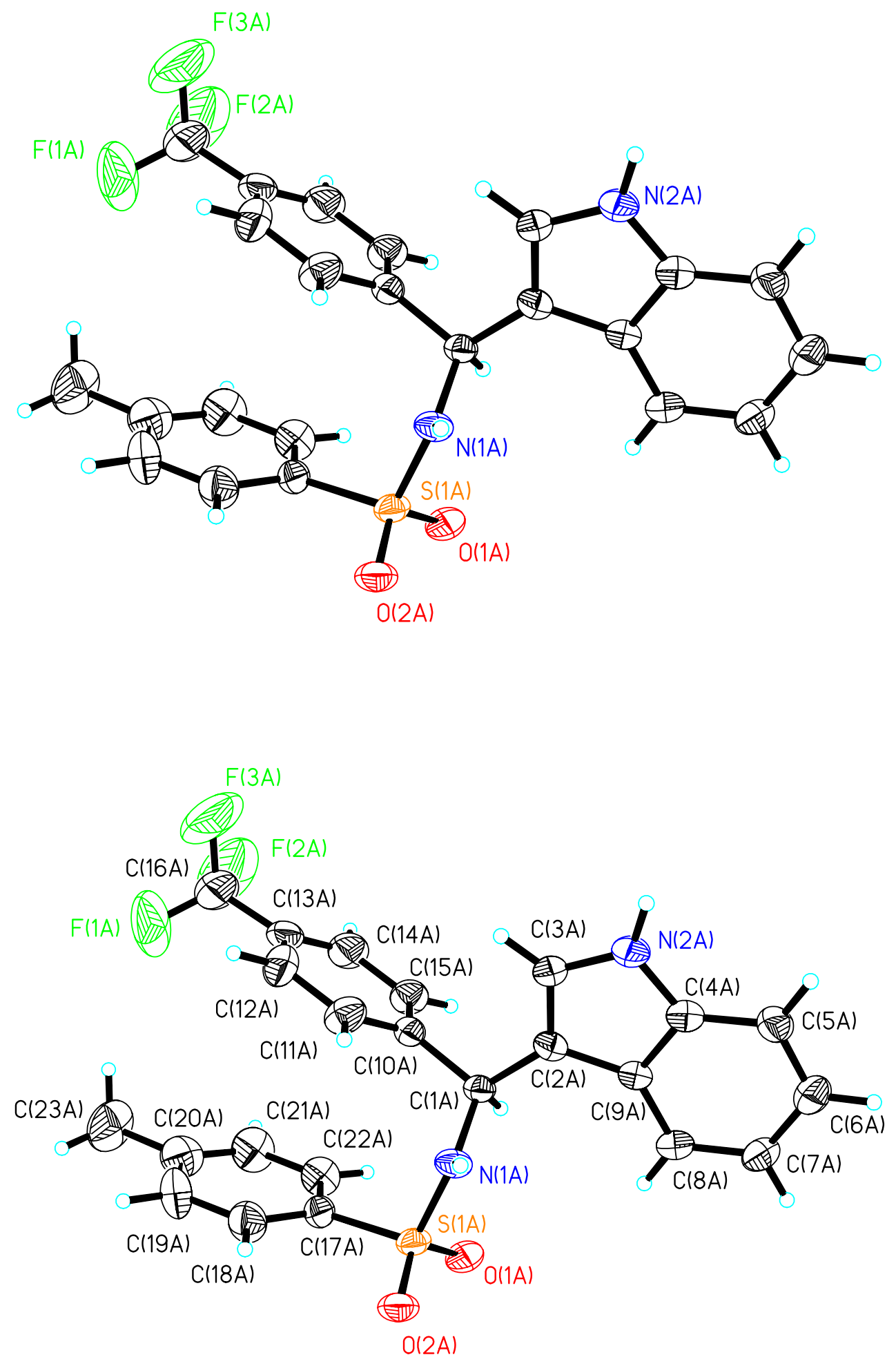

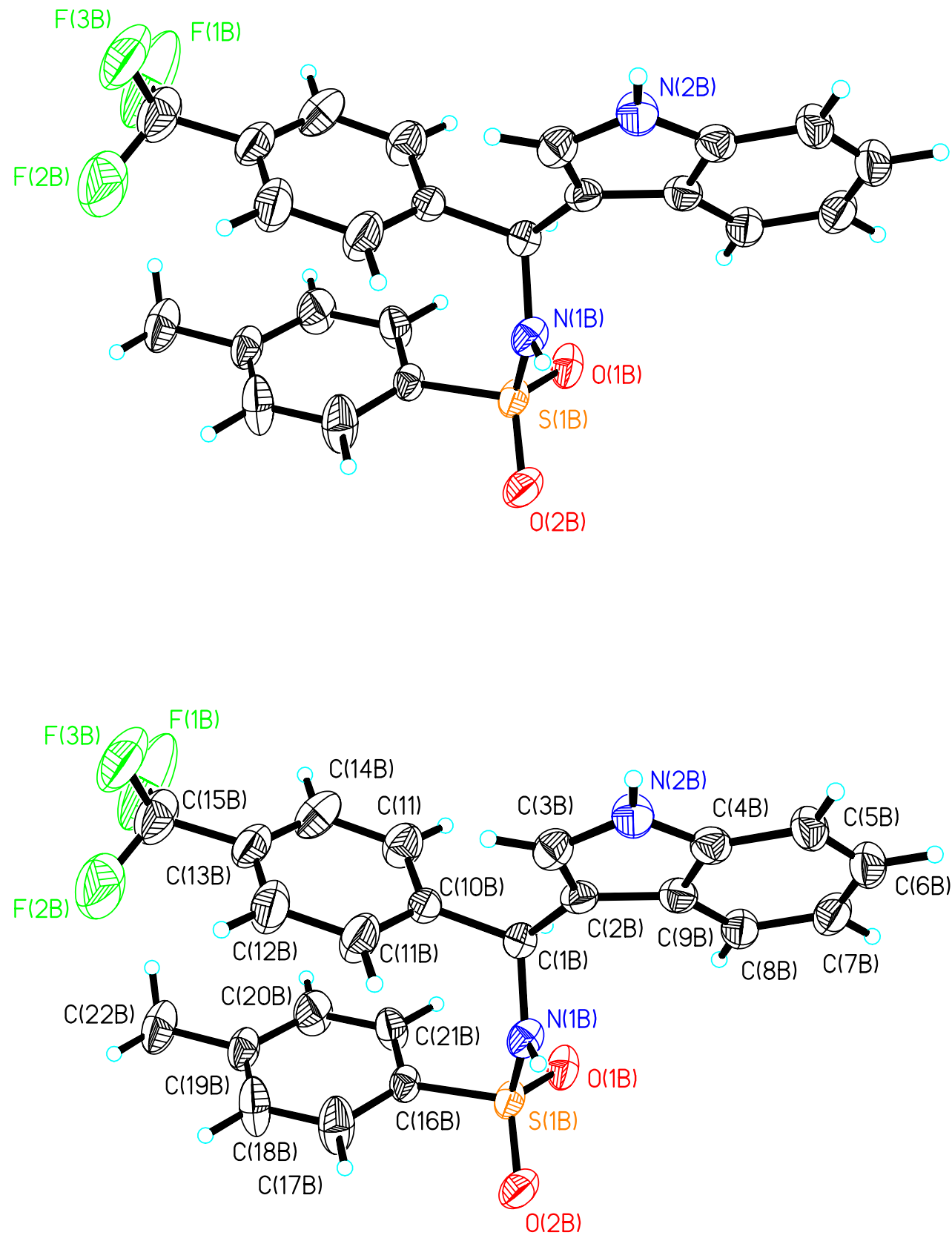


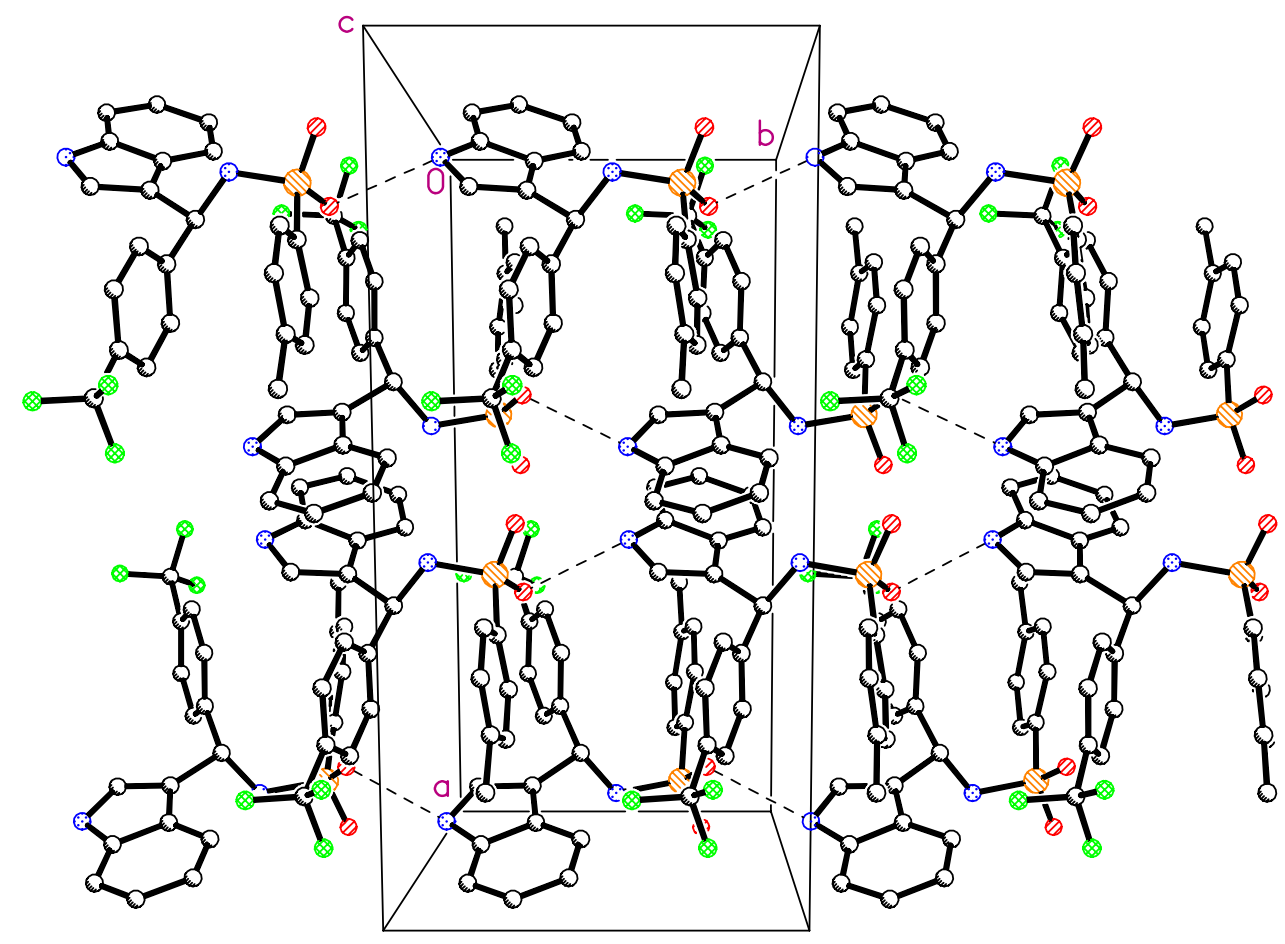

The last picture is the packing diagram along the c-axis which shows the best diagram of the hydrogen bonding within the crystal, dotted lines. 
Table 1. Crystal data and structure refinement for ld11t.

Identification code

ld11t

Empirical formula

C23 H19 F3 N2 O2 S

Formula weight

444.46

Temperature

193(2) K

Wavelength

$0.71073 \AA$

Crystal system

Monoclinic

Space group

$\mathrm{C} 2$

Unit cell dimensions

$\mathrm{a}=18.346(4) \AA$

$\alpha=90^{\circ}$.

$\mathrm{b}=8.6134(16) \AA$

$\beta=105.690(3)^{\circ}$.

$\mathrm{c}=27.741(6) \AA$

$\gamma=90^{\circ}$.

Volume

4220.4(14) $\AA^{3}$

Z

Density (calculated)

8

$1.399 \mathrm{Mg} / \mathrm{m}^{3}$

Absorption coefficient

$0.202 \mathrm{~mm}^{-1}$

$\mathrm{F}(000)$

1840

Crystal size

$0.14 \times 0.10 \times 0.04 \mathrm{~mm}^{3}$

Theta range for data collection

0.76 to $27.93^{\circ}$.

Index ranges

$-22<=\mathrm{h}<=23,-11<=\mathrm{k}<=8,-36<=\mathrm{l}<=27$

Reflections collected

13935

Independent reflections

$8981[\mathrm{R}(\mathrm{int})=0.0333]$

Completeness to theta $=27.93^{\circ}$

Absorption correction

$99.0 \%$

Empirical

Max. and min. transmission

Refinement method

0.9920 and 0.9722

Full-matrix least-squares on $\mathrm{F}^{2}$

Data / restraints / parameters

$8981 / 1 / 561$

Goodness-of-fit on $\mathrm{F}^{2}$

1.035

Final $\mathrm{R}$ indices $[\mathrm{I}>2 \operatorname{sigma}(\mathrm{I})]$

$\mathrm{R} 1=0.0546, \mathrm{wR} 2=0.1367$

$\mathrm{R}$ indices (all data)

$\mathrm{R} 1=0.0893, \mathrm{wR} 2=0.1531$

Absolute structure parameter

$0.03(7)$

Largest diff. peak and hole 
Determination of the absolute configuration of $(+)-3 \mathrm{Aj}$ by the modified Mosher's method: ${ }^{7}$

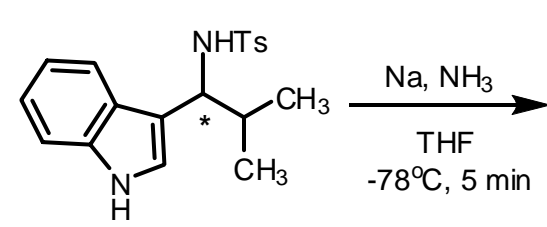

$(+)-3 A j$

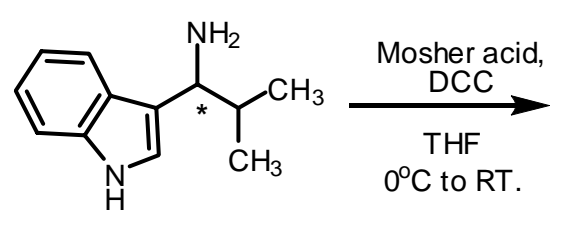

Friedel-Crafts reaction adduct $(+)-\mathbf{3 A j}(10 \mathrm{mg}, 0.292 \mathrm{mmol})$ was subjected to the deprotection of Ts group procedure as described in detail page $\mathrm{S}-14$. The crude free amine $(3.0 \mathrm{mg}, 0.016 \mathrm{mmol})$ and $(R)$-MTPA acid $(3.8 \mathrm{mg}, 0.0176 \mathrm{mmol})$ dissolved in anhydrous $\mathrm{CH}_{2} \mathrm{Cl}_{2}(0.5 \mathrm{~mL})$ at room temperature. DCC $(3.5 \mathrm{mg}, 0.0176 \mathrm{mmol})$ was added in one portion. The resulting mixture was stirred at room temperature for $40 \mathrm{~min}$. The white suspension was filtered through a short plug of silica gel. The filtrate was concentrated and the residue was subjected to purification on TLC plate (EMD Silica gel $60 \mathrm{~F}_{254}$, petroleum ether/EtOAc: $\left.3 / 1\right)$ to afford $(R)$-MTPA amide $(R, R)-\mathbf{9 A j}(4.3 \mathrm{mg}$, $85 \%$ yield). Following the above procedure, the $(S)$-MTPA amide $(R, S)$-9Aj was prepared.

The absolute configuration of the primary amine was determined as $R$ with its Mosher's amides derivatives based on the Modified Mosher's method developed by Kakisawa and co-workers (vide infra).<smiles>CC(C)C=[N+]=[N-]</smiles> 
Table ${ }^{1} \mathrm{H}$ NMR Spectral Data for the MTPA Amides $\left(\delta, \mathrm{CDCl}_{3}\right)$

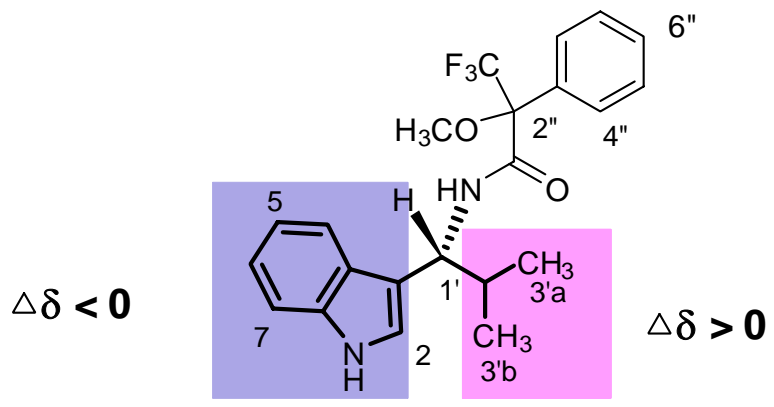

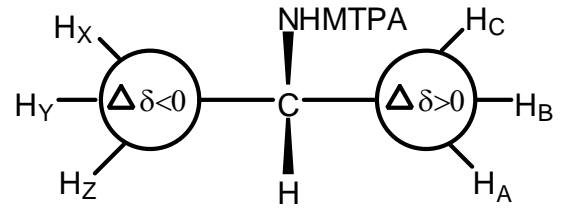

Model

\begin{tabular}{llll}
\hline protons & $\begin{array}{c}(S)-\text { MTPA amide } \\
(R, S)-9 \mathrm{Aj}\end{array}$ & $\begin{array}{c}(R)-\mathrm{MTPA} \text { amide } \\
(R, R)-9 \mathrm{Aj}\end{array}$ & $\Delta \delta=\delta_{S^{-}} \delta_{R}(\mathrm{ppm})$ \\
\hline $1-\mathrm{H}$ & $8.052(\mathrm{~s})$ & $8.102(\mathrm{~s})$ & -0.050 \\
$2-\mathrm{H}$ & $7.050(\mathrm{~s})$ & $7.098(\mathrm{~s})$ & -0.048 \\
$4-\mathrm{H}$ & $7.564(\mathrm{~d}, 8.0)$ & $7.634(\mathrm{~d}, 8.0)$ & -0.070 \\
$5-\mathrm{H}$ & $7.067(\mathrm{t}, 7.2)$ & $7.118(\mathrm{t}, 7.2)$ & -0.051 \\
$6-\mathrm{H}$ & $7.170(\mathrm{t}, 8.0)$ & $7.202(\mathrm{t}, 8.0)$ & -0.052 \\
$7-\mathrm{H}$ & $7.372(\mathrm{~d}, 8.0)$ & $7.367(\mathrm{~d}, 8.0)$ & +0.005 \\
1'-H & $5.148(\mathrm{t}, 8.8)$ & $5.135(\mathrm{t}, 8.8)$ & +0.013 \\
$2 '-\mathrm{H}$ & $2.394(\mathrm{~m})$ & $2.322(\mathrm{~m})$ & +0.072 \\
$3 ' \mathrm{a}-\mathrm{H}$ & $1.079(\mathrm{~d}, 6.4)$ & $0.923(\mathrm{~d}, 6.0)$ & +0.156 \\
3'b-H & $0.991(\mathrm{~d}, 6.8)$ & $0.907(\mathrm{~d}, 6.8)$ & +0.084 \\
CONH & $7.164(\mathrm{~d}, 8.8)$ & $6.937(\mathrm{~d}, 9.2)$ & \\
$2 "-\mathrm{OCH}{ }_{3}$ & $3.326(\mathrm{~s})$ & $3.257(\mathrm{~s})$ & \\
$4 ", 5 ", 6 "-\mathrm{H}$ & $7.329(\mathrm{~d}, 8.0,2 \mathrm{H})$ & $7.408(\mathrm{~m}, 3 \mathrm{H})$ & \\
& $7.293(\mathrm{t}, 7.2,1 \mathrm{H})$ & $7.606(\mathrm{~m}, 2 \mathrm{H})$ & \\
& $7.208(\mathrm{t}, 7.2,1 \mathrm{H})$ & & \\
\hline
\end{tabular}

Mosher's $\underline{\text { Amide }}$

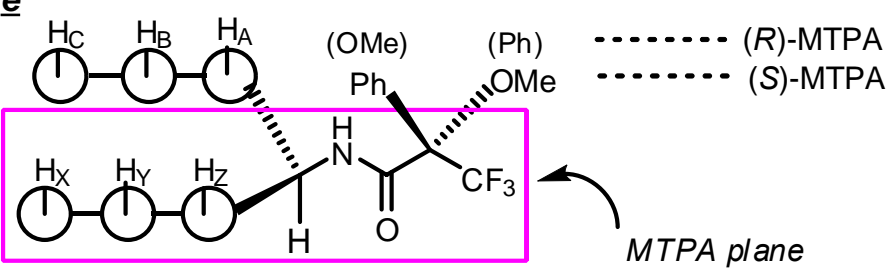




\section{Reference}

(1) (a) Chemla, F.; Hebbe, V.; Normant, J.-F. Synthesis 2000, 75; (b) Ooi, T.; Uematus, Y.; Maruoka, K. J. Am. Chem. Soc. 2006, 128; 2548.

(2) Sivakumar, A. V.; Babu, G. S.; Bhat, S. V. Tetrahedron: Asymmetry 2001, 12, 1095.

(3) Jennings, W. B.; Lovely, C. Tetrahedron 1991, 47, 5561.

(4) (a) Li, H.; Wang, Y.; Tang, L.; Deng, L. J. Am. Chem. Soc. 2004, 126, 9906; (b) Li, H.; Wang, Y.; Tang, L.; Wu, F.; Liu, X.; Guo, C.; Foxman, B. M.; Deng L. Angew. Chem.Int. Ed. 2005, 44, 105; (c) Liu, X.; Li, H.; Deng, L. Org. Lett. 2005, 7, 167; (d) Li, H.; Wang, B.; Deng, L. J. Am. Chem. Soc. 2006, 128, 732.

(5) (a) Li, B.; Jiang, L.; Liu, M.; Chen, Y.; Ding, L.; Wu, Y. Synlett 2005, 4, 603; (b) Vakulya, B.; Varga, S.; Csámpai, A.; Soós, T. Org Lett. 2005, 7, 1967.

(6) Schultz, A. G.; McCloskey, P. J.; Court, J. J. J. Am. Chem. Soc. 1987, 109, 6493

(7) Kusumi, T.; Fukushima, T.; Ohtani, I.; Kakisawa, H. Tetrahedron Lett. 1991, 32, 2939. 
HPLC Conditions: Daicel chiralpak OD, Hexanes:IPA, 60:40 1.0 mL/min, $\lambda 254 \mathrm{~nm}$

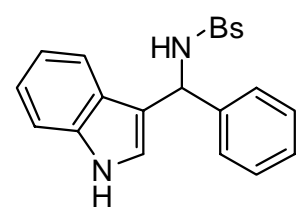

Racemic 3Aa
(-)-3Aa 94\% ee

Product obtained with 7
(+)-3Aa $92 \%$ ee

Product obtained with 6
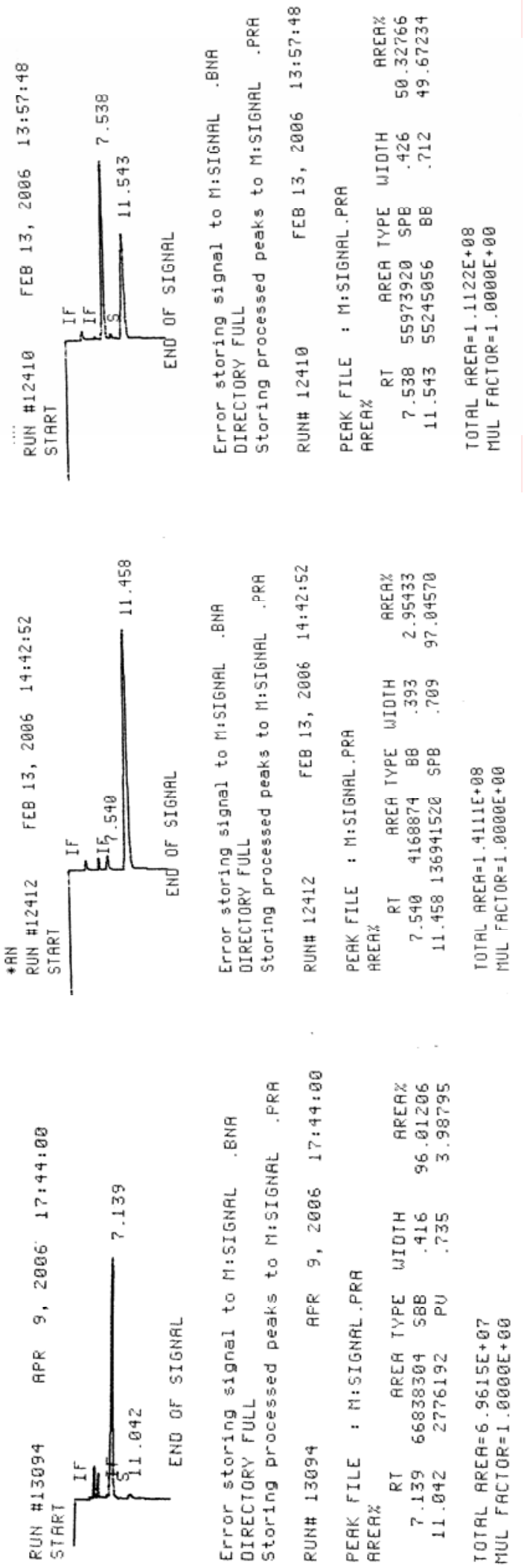
HPLC Conditions: Daicel chiralpak OD, Hexanes:IPA, 60:40 1.0 mL/min, $\lambda 220 \mathrm{~nm}$

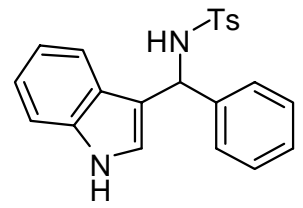

Racemic 3Ab

(-)-3Ab 94\% ee

Product obtained with 7

(+)-3Ab $94 \%$ ee

Product obtained with 6
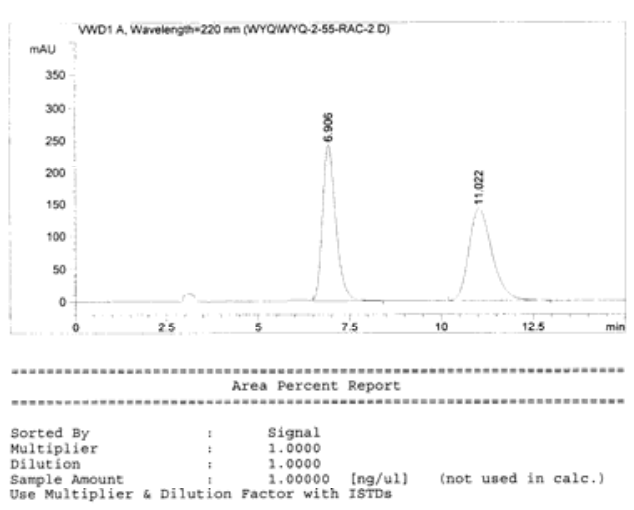

Signal 1: VWD1 A, kavelength-220 nm

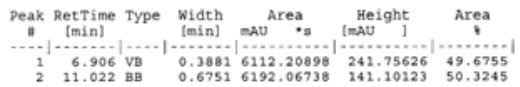
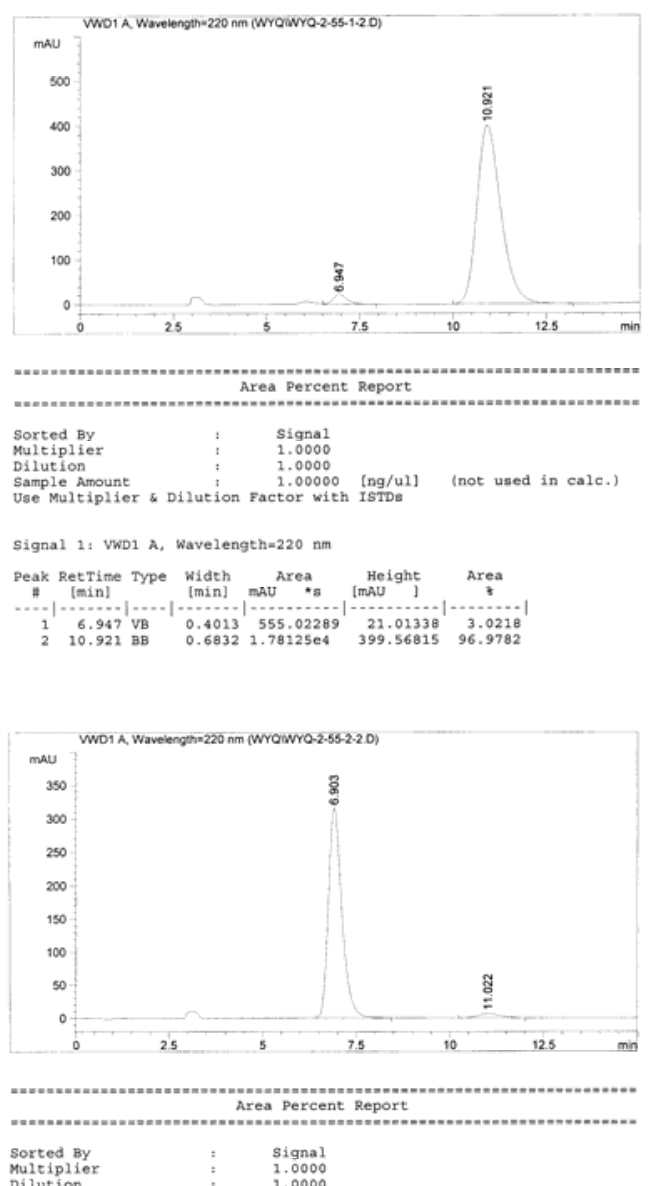

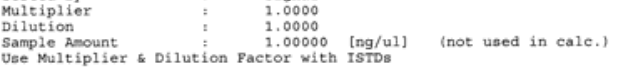

Signal 1: VWD1 A, Wavelengthe $220 \mathrm{~nm}$

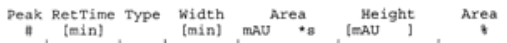

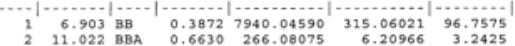


HPLC Conditions: Daicel chiralpak OD, Hexanes:IPA, 70:30 1.0 mL/min, $\lambda 254 \mathrm{~nm}$<smiles>CC(C)(C)NC(c1ccccc1)c1c[nH]c2cc(Cl)ccc12</smiles>

Racemic 3Ba

(-)-3Ba $90 \%$ ee

Product obtained with 7

$(+)-3$ Ba $95 \%$ ee

Product obtained with 6

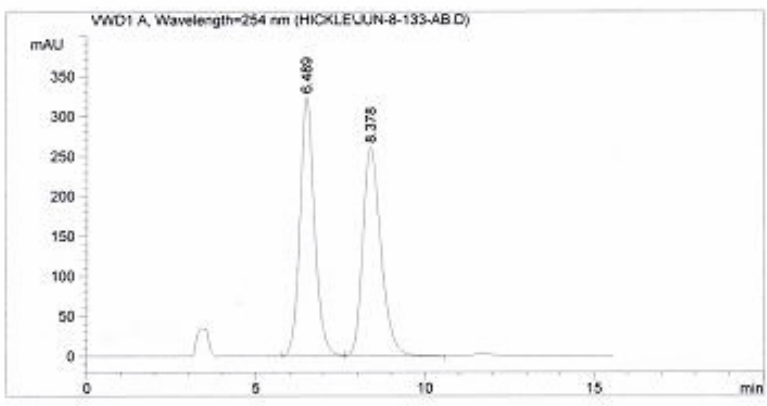

Signa1 1: VWD1 A, wavelength $=254 \mathrm{~nm}$

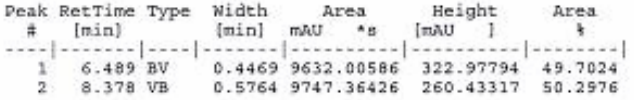

Totale : $\quad 1.93796 \mathrm{ed} \quad 583.41110$

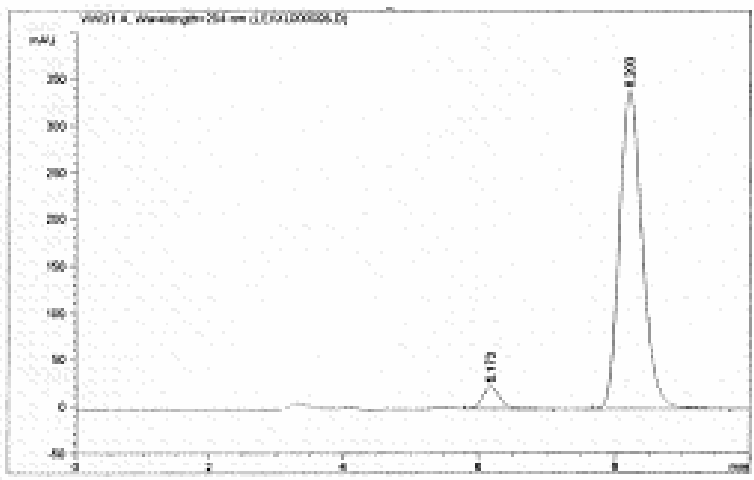

Signat 1i: wol A, kavelesgeh*254 tom

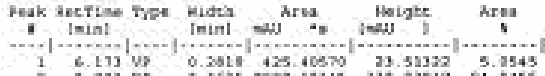

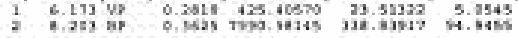

Desela . 2416.38715 3ez.39203

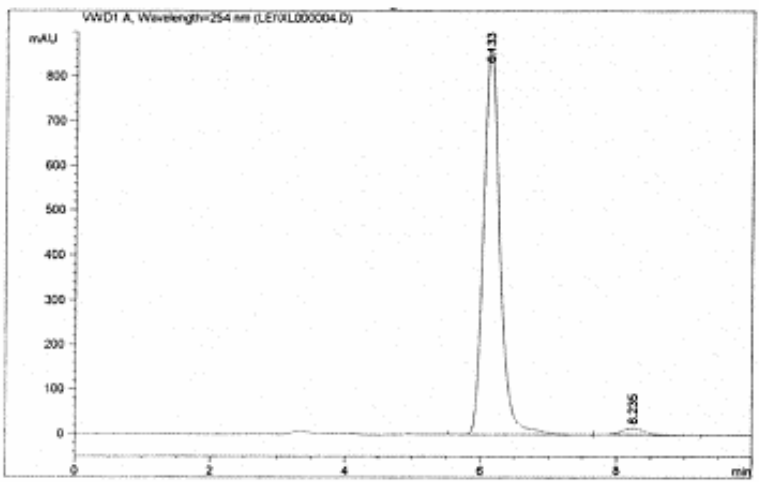

Signal if WDi A, Wavelength=254 nn

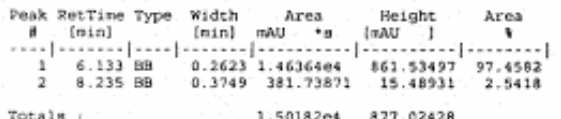

7otalg f $1.50182 \mathrm{et} \quad 877.02428$ 
HPLC Conditions: Daicel chiralpak OD, Hexanes:IPA, 60:40 1.0 mL/min, $\lambda 254$ nm<smiles>O=C(NC(c1ccccc1)c1c[nH]c2cc(Br)ccc12)c1ccccc1</smiles>

Racemic 3Ca
(-)-3Ca 95\% ee

Product obtained with 7

(+)-3Ca $95 \%$ ee

Product obtained with 6
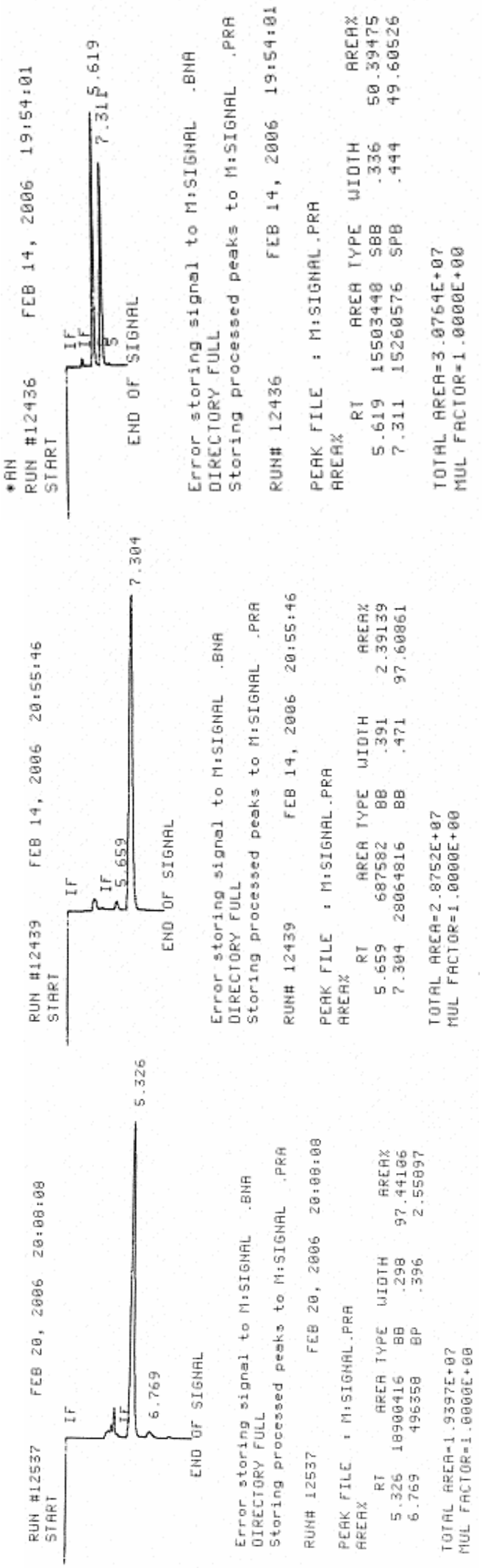
HPLC Conditions: Daicel chiralpak OD, Hexanes:IPA, 60:40 1.0 mL/min, $\lambda 254 \mathrm{~nm}$<smiles>COc1ccc2c(C(NC(=O)c3ccccc3)c3ccccc3)c[nH]c2c1</smiles>

Racemic 3Da
(-)-3Da 92\% ee

Product obtained with 7

(+)-3Da 93\% ee

Product obtained with 6
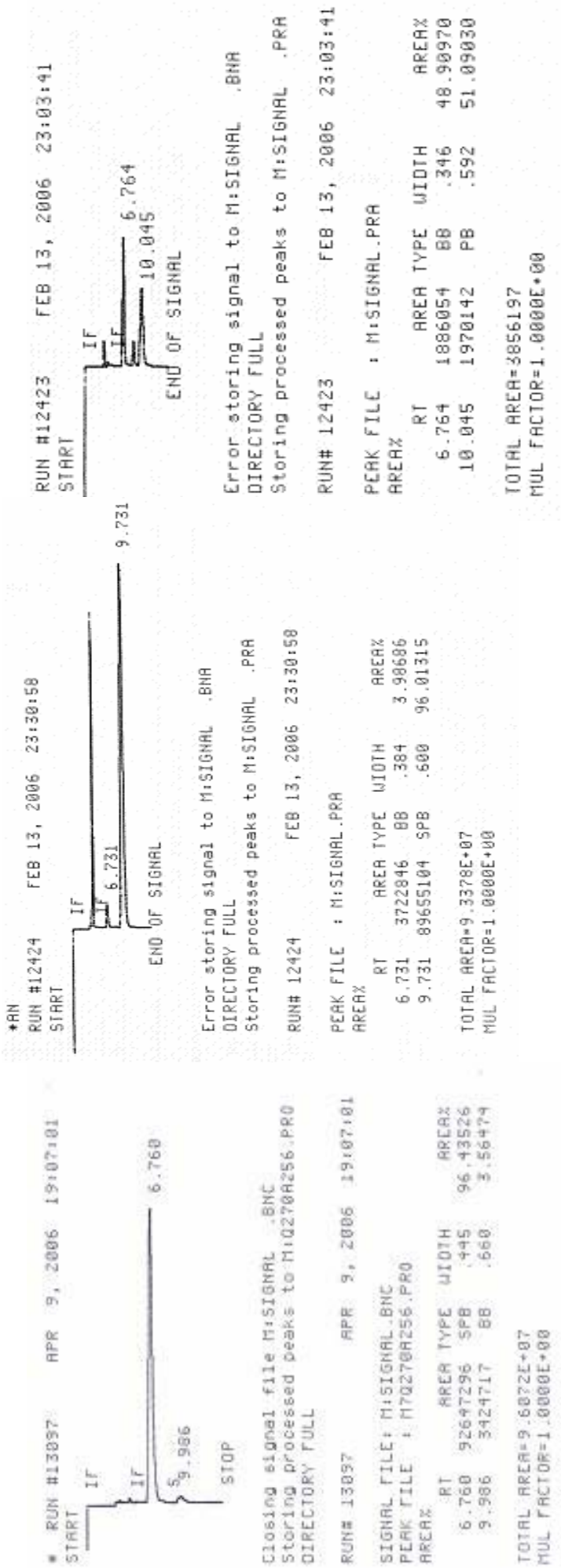
HPLC Conditions: Daicel chiralpak OD, Hexanes:IPA, 60:40 1.0 mL/min, $\lambda 254 \mathrm{~nm}$

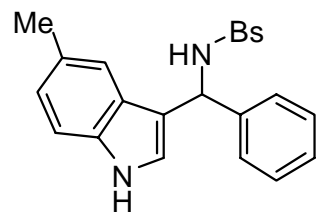

Racemic 3Ea

(-)-3Ea $89 \%$ ee

Product obtained with 7

(+)-3Ea $90 \%$ ee

Product obtained with $\mathbf{6}$
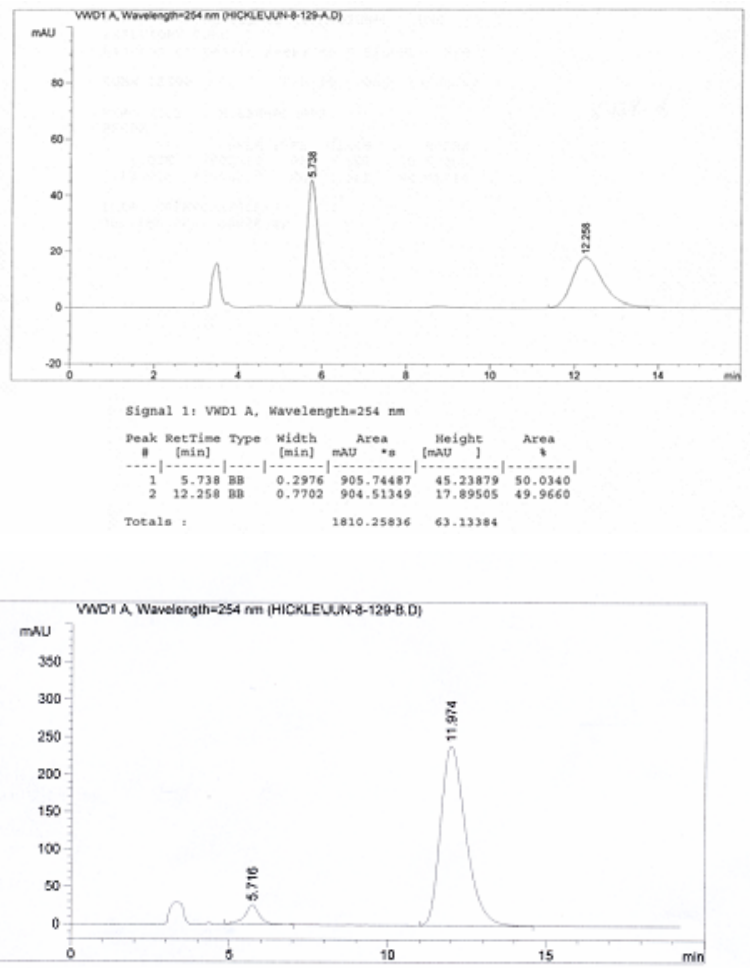

Signal 1: VWD1 $\mathrm{A}$, Wavelength $=254 \mathrm{nh}$

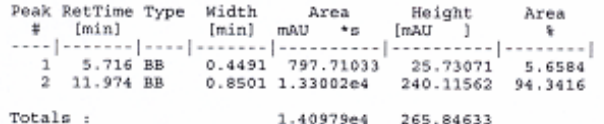

Totale : $\quad 1.40979 \mathrm{e} \quad 265.84633$

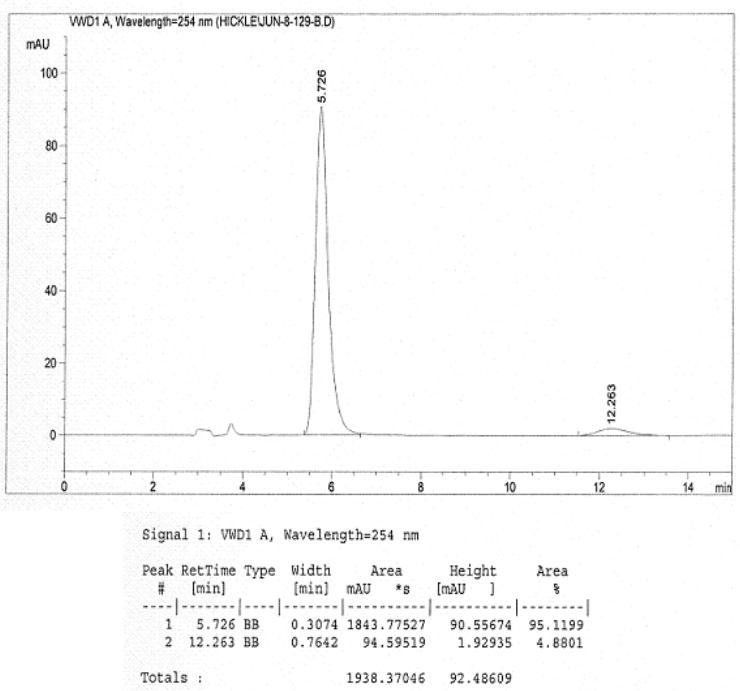


HPLC Conditions: Daicel chiralpak OD, Hexanes:IPA, 70:30 1.0 mL/min, $\lambda 220 \mathrm{~nm}$

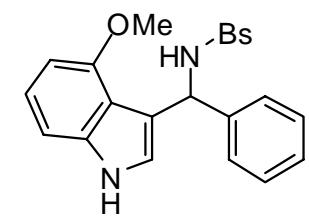

Racemic 3Fa

(-)-3Fa 91\% ee

Product obtained with 7

(+)-3Fa $90 \%$ ee

Product obtained with 6
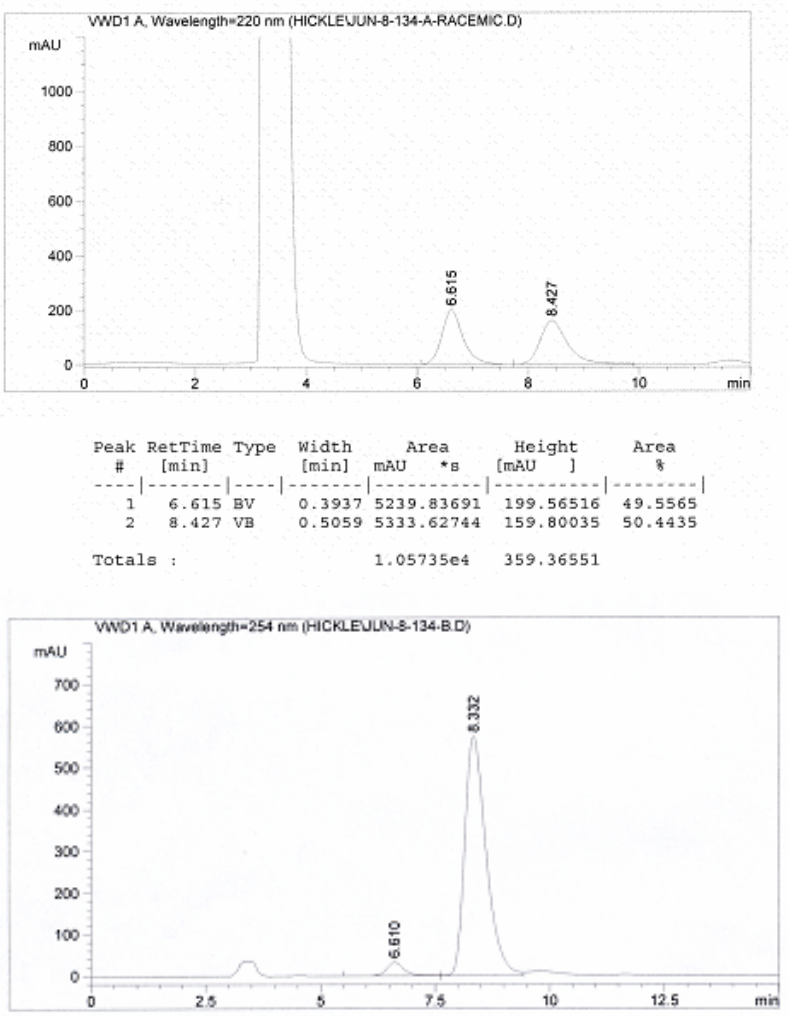

Signa1 1: WuD1 $\mathrm{A}$, Wavelength=254 $\mathrm{nm}$

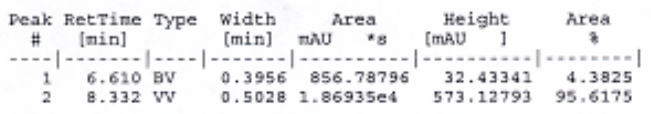

Totals : $1.95503 e 4 \quad 605.56134$

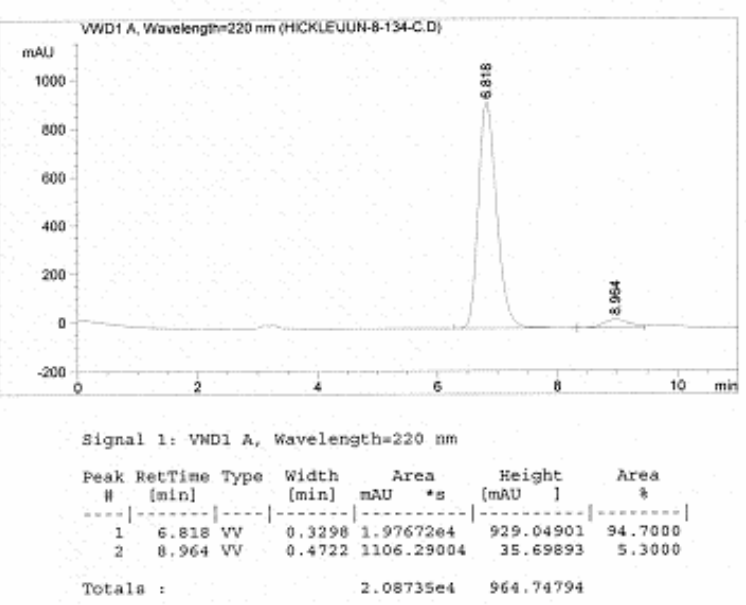


HPLC Conditions: Daicel chiralpak OD, Hexanes:IPA, 60:40 1.0 mL/min, $\lambda 220 \mathrm{~nm}$

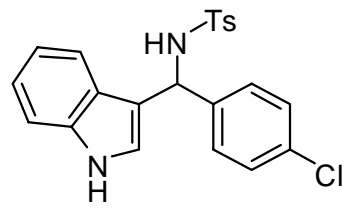

Racemic 3Ac

(-)-3Ac 94\% ee

Product obtained with 7

$(+)-3$ Ac $93 \%$ ee

Product obtained with 6
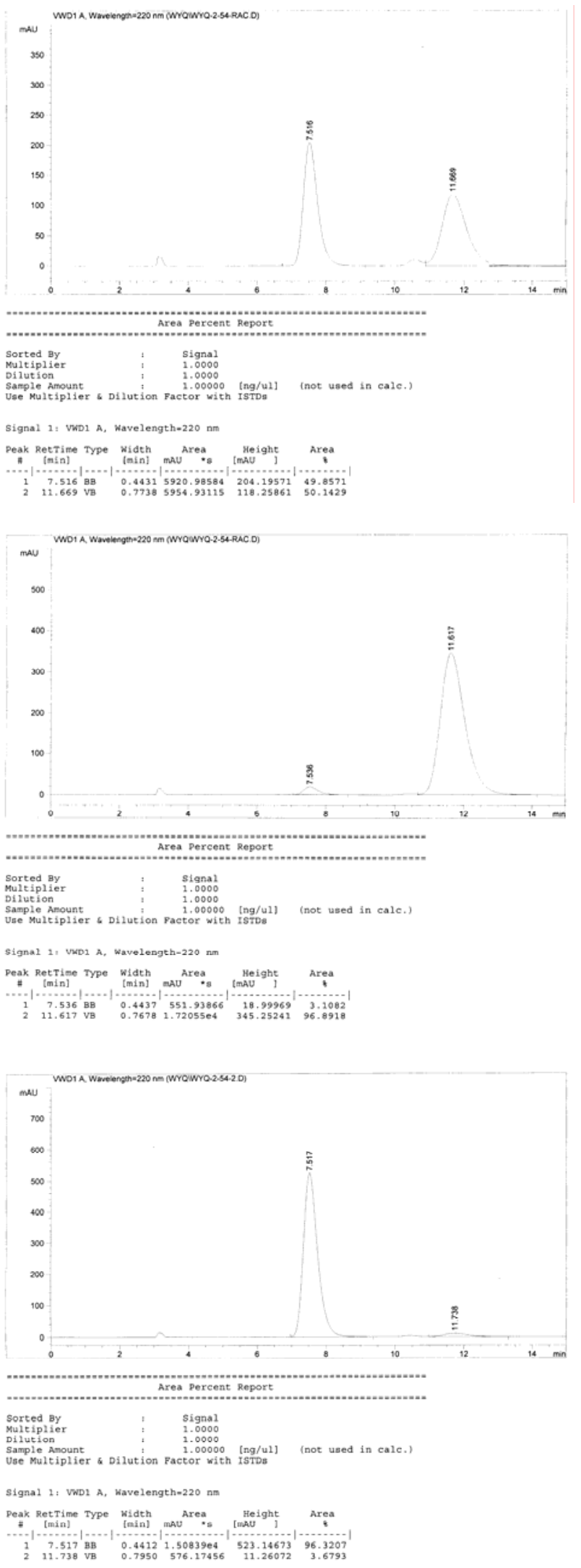
HPLC Conditions: Daicel chiralpak OD, Hexanes:IPA, 60:40 1.0 mL/min, $\lambda 220 \mathrm{~nm}$

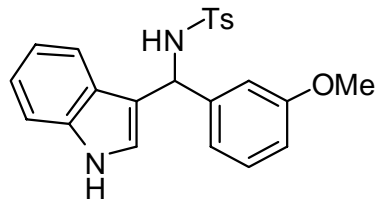

Racemic 3Ad

(-)-3Ad $92 \%$ ee

Product obtained with 7

(+)-3Ad $90 \%$ ee

Product obtained with 6
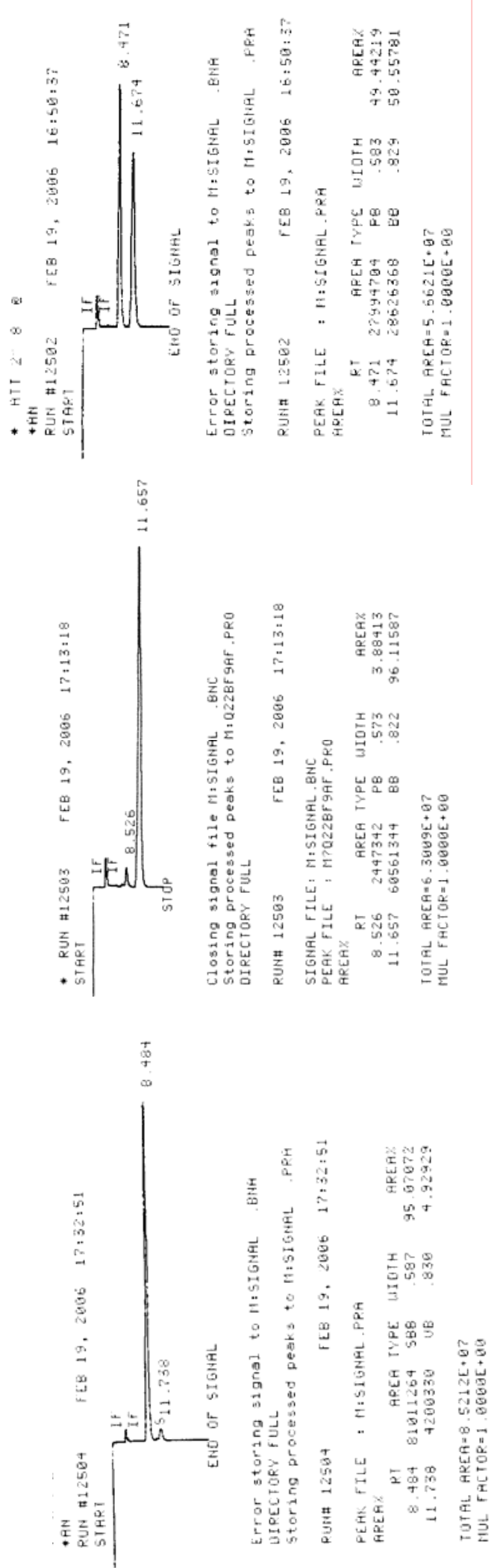
HPLC Conditions: Daicel chiralpak OD, Hexanes:IPA, 60:40 1.0 mL/min, $\lambda 220 \mathrm{~nm}$

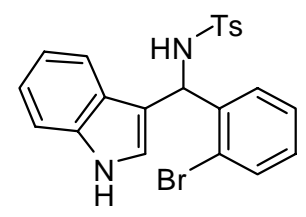

Racemic 3Ae

(-)-3Ae 93\% ee

Product obtained with 7

$(+)$-3Ae $93 \%$ ee

Product obtained with 6
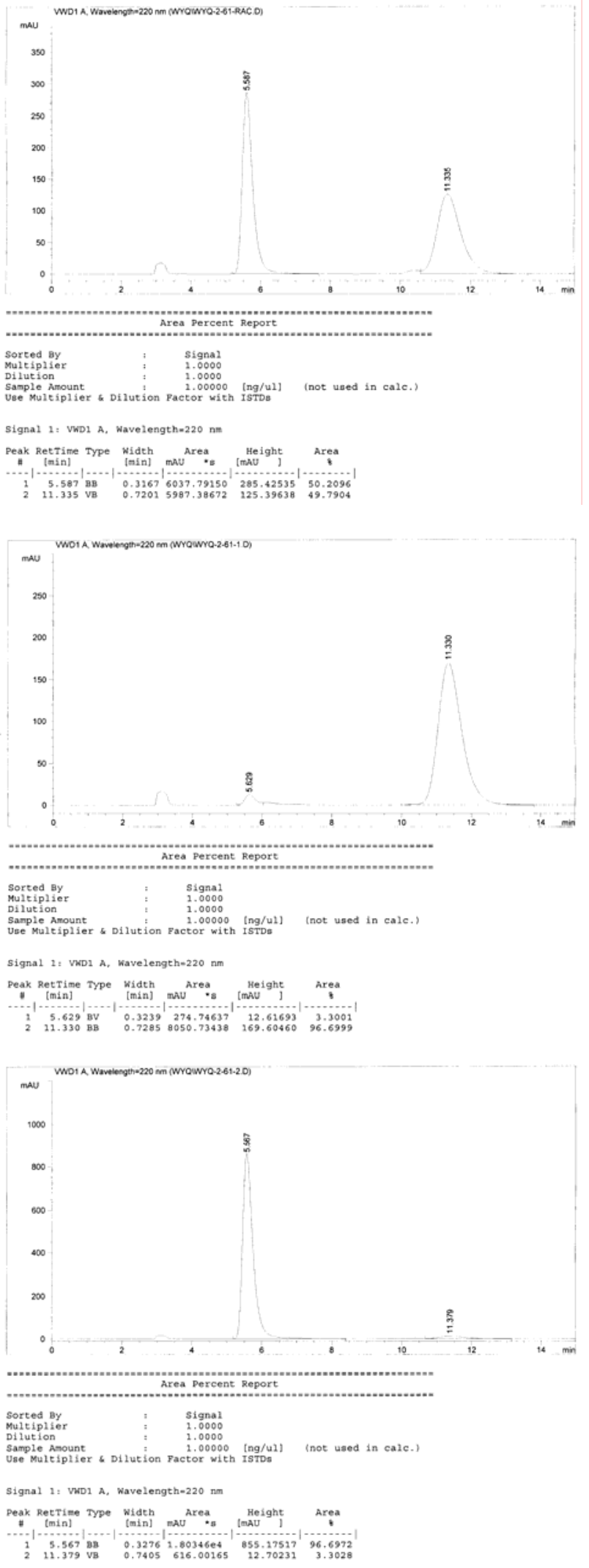
HPLC Conditions: Daicel chiralpak OD, Hexanes:IPA, 60:40 1.0 mL/min, $\lambda 220 \mathrm{~nm}$

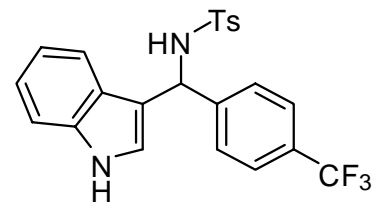

Racemic 3Af
(-)-3Af 96\% ee

Product obtained with 7

(+)-3Af $97 \%$ ee

Product obtained with 6
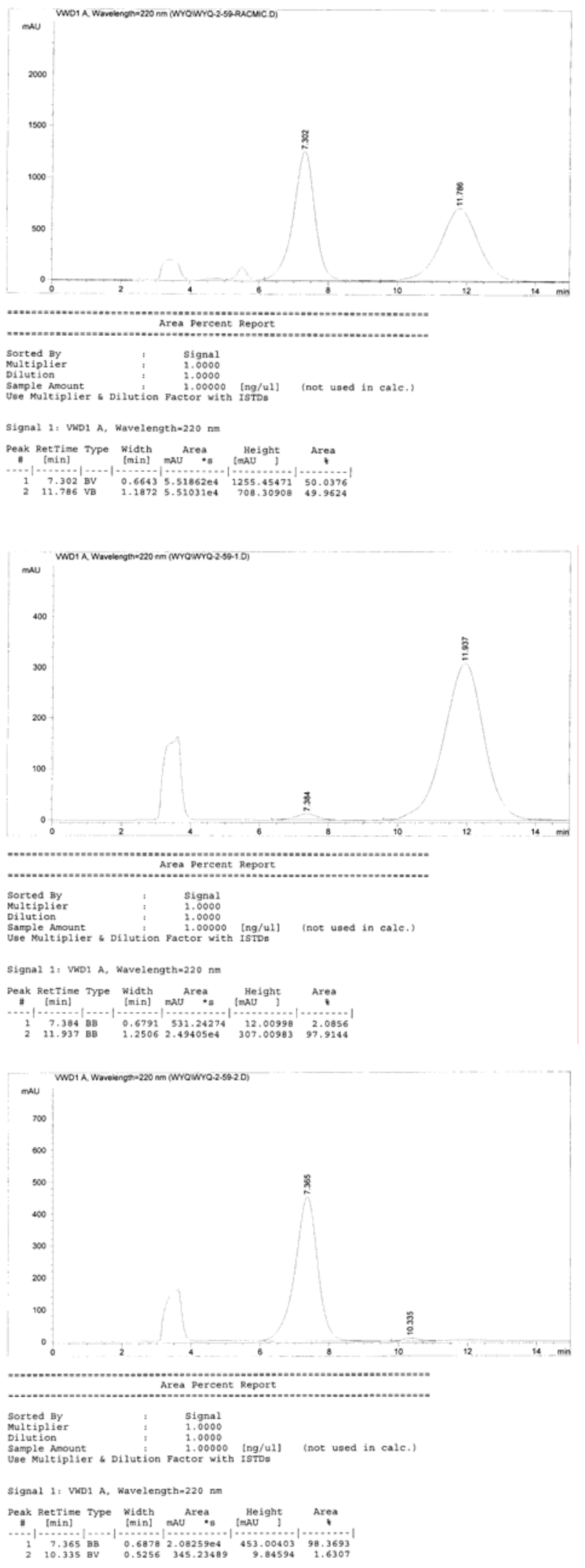
HPLC Conditions: Daicel chiralpak OD, Hexanes:IPA, 60:40 1.0 mL/min, $\lambda 220 \mathrm{~nm}$

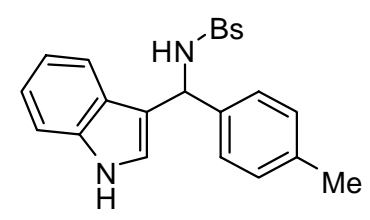

Racemic 3Ag

(-)-3Ag 92\% ee

Product obtained with 7

(+)-3Ag $94 \%$ ee

Product obtained with 6
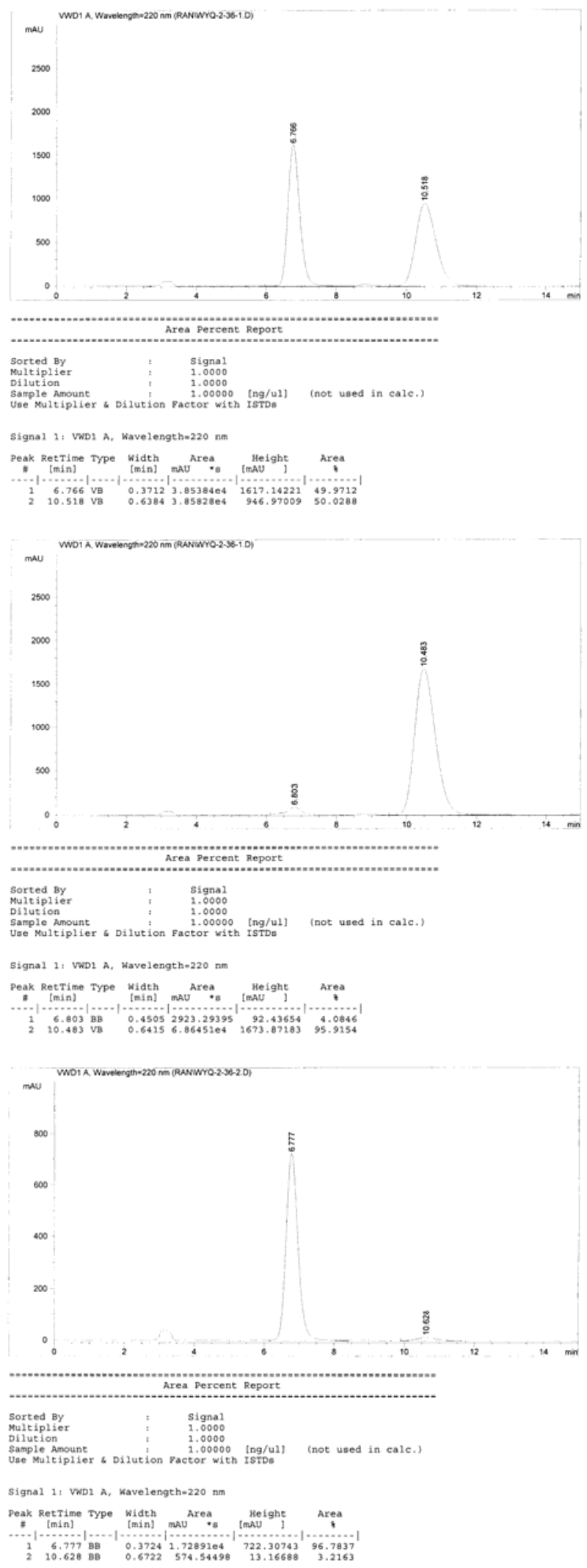
HPLC Conditions: Daicel chiralpak OD, Hexanes:IPA, 60:40 1.0 mL/min, $\lambda 220 \mathrm{~nm}$

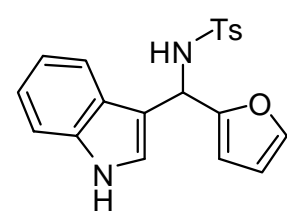

Racemic 3Ah
(-)-3Ah $96 \%$ ee

Product obtained with 7
(+)-3Ah 94\% ee

Product obtained with 6
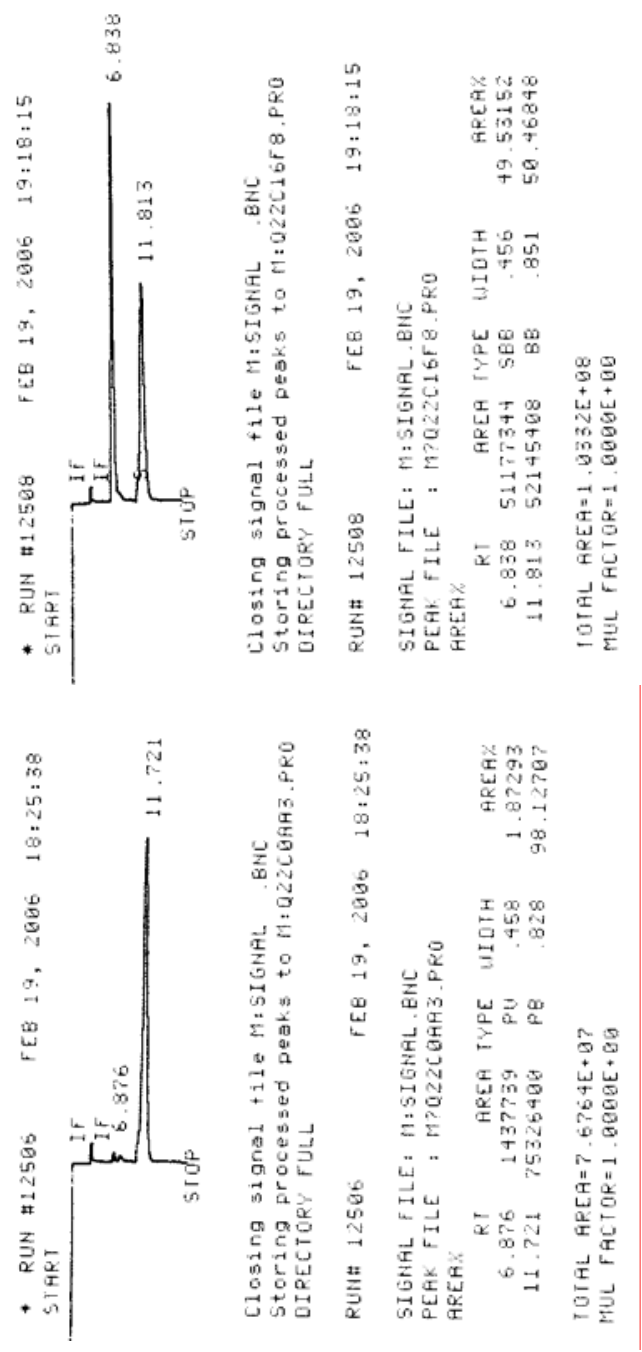

$\stackrel{\varphi}{\mathscr{Q}}$

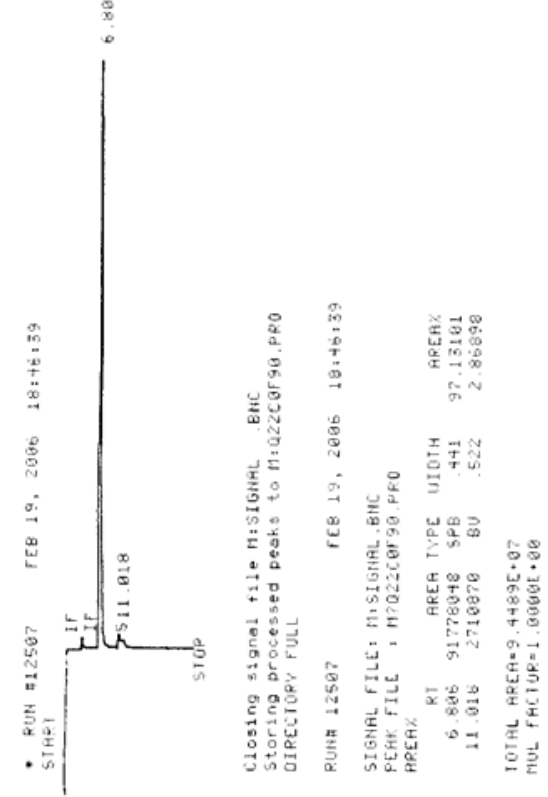


HPLC Conditions: Daicel chiralpak OD, Hexanes:IPA, 70:30 1.0 mL/min, $\lambda 254 \mathrm{~nm}$

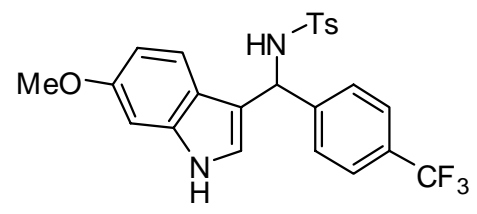

Racemic 3Df

(-)-3Df 95\% ee

Product obtained with 7

(+)-3Df $94 \%$ ee

Product obtained with 6
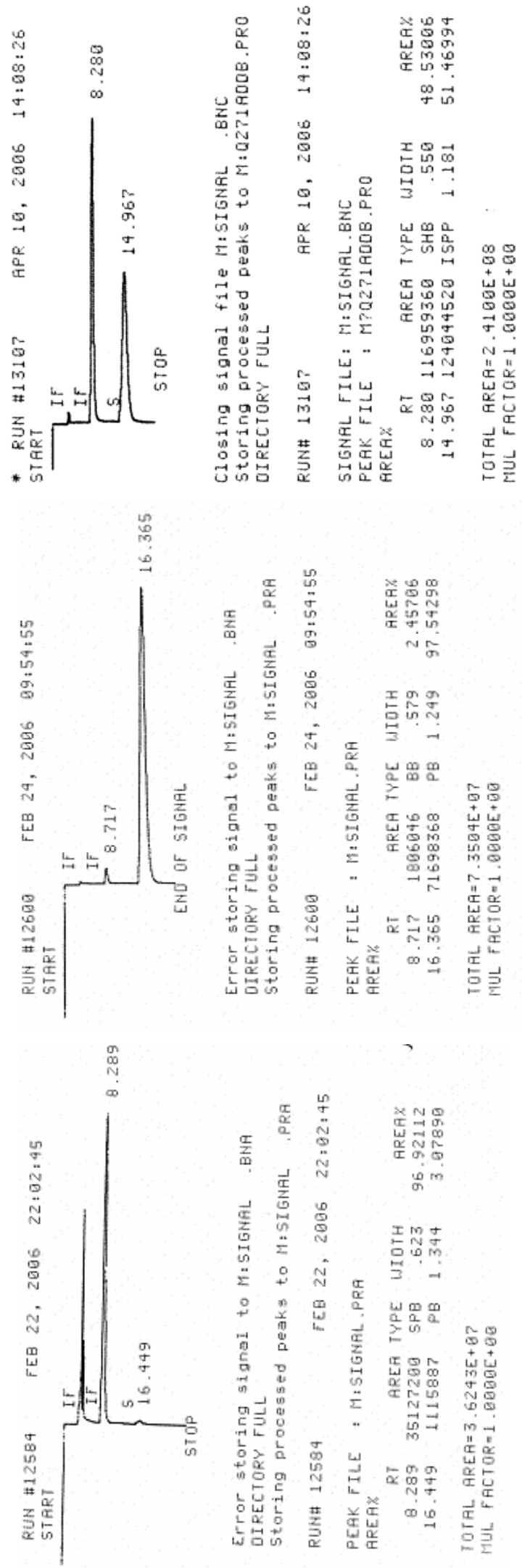
HPLC Conditions: Daicel chiralpak OD, Hexanes:IPA, 70:30 1.0 mL/min, $\lambda 254 \mathrm{~nm}$<smiles>COc1cccc(C(NC(F)(F)F)c2c[nH]c3cc(Br)ccc23)c1</smiles>

Racemic 3Cd

(-)-3Cd 86\% ee

Product obtained with 7

$(+)-3 C d 83 \%$ ee

Product obtained with 6
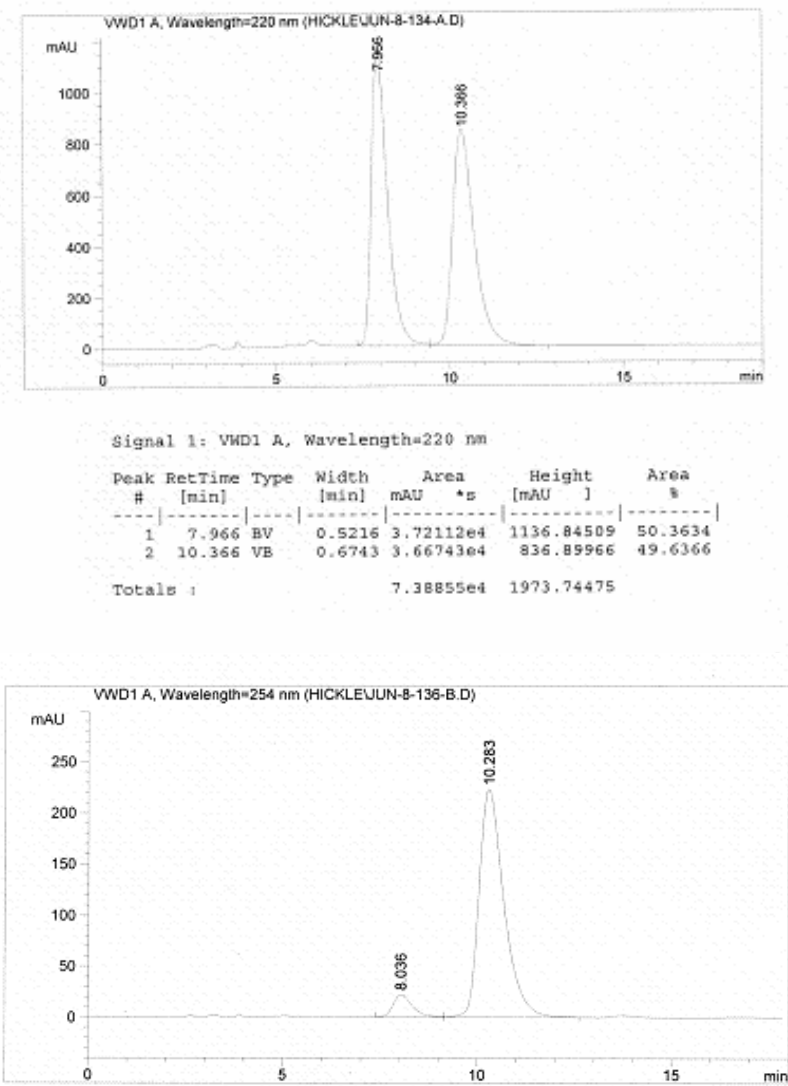

Signal 1: VWD1 $A$, Wavelength $=254 \mathrm{~nm}$

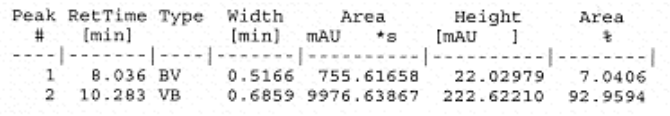

Totals :

$1.07323 \mathrm{e} 4 \quad 244.65189$

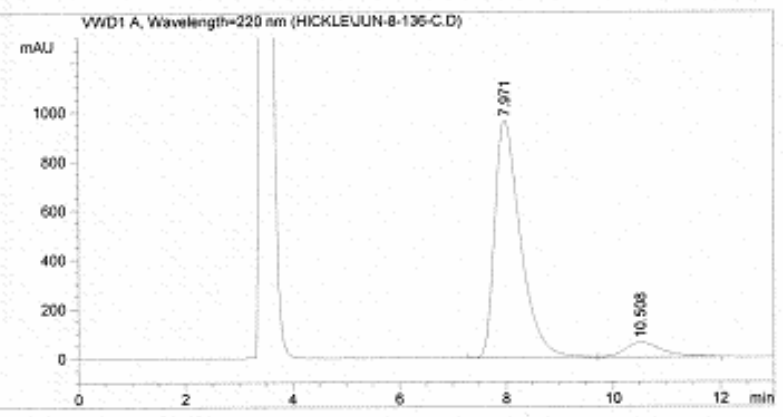

Signal 1: WWD1 $\mathrm{A}$, wavelength=220 nm

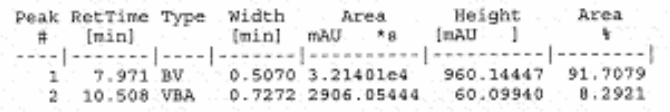

Totals :

$3.5046204 \quad 2020.24387$ 
HPLC Conditions: Daicel chiralpak OD, Hexanes:IPA, 60:40 1.0 mL/min, $\lambda 220 \mathrm{~nm}$

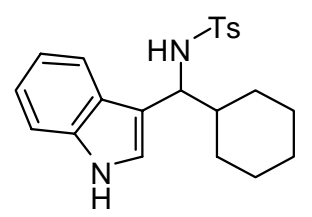

Racemic 3Ai
(-)-3Ai 94\% ee

Product obtained with 7

$(+)-3 \mathbf{A i} 93 \%$ ee

Product obtained with 6
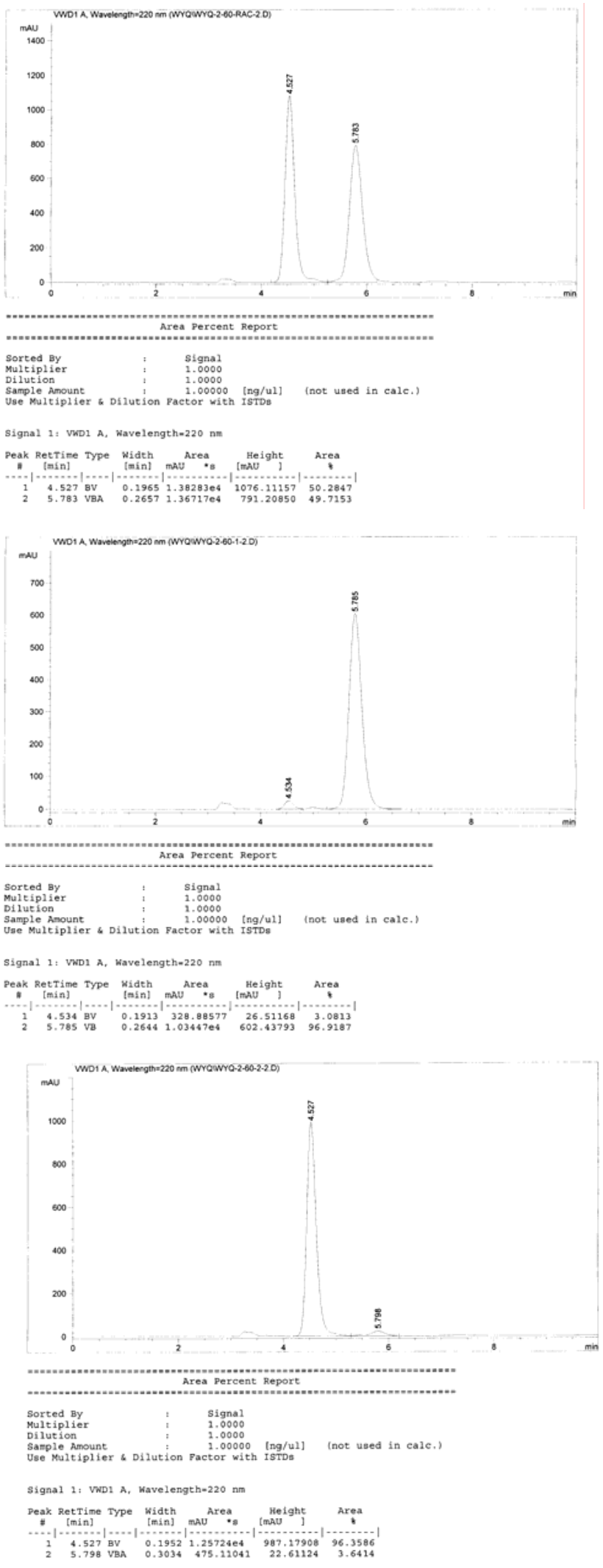
HPLC Conditions: Daicel chiralpak OD, Hexanes:IPA, 80:20 1.0 mL/min, $\lambda 220 \mathrm{~nm}$

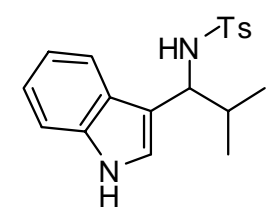

Racemic 3Aj

(-)-3Aj 94\% ee

Product obtained with 7

(+)-3Aj $94 \%$ ee

Product obtained with 6
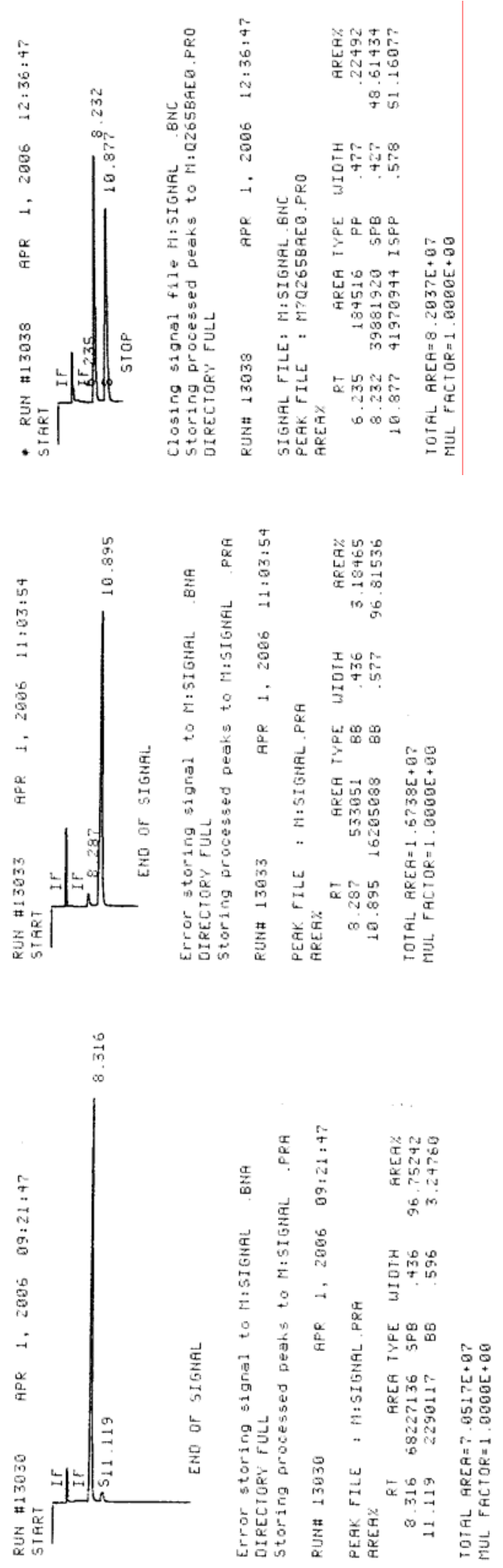
HPLC Conditions: Daicel chiralpak OD, Hexanes:IPA, 60:40 1.0 mL/min, $\lambda 220 \mathrm{~nm}$

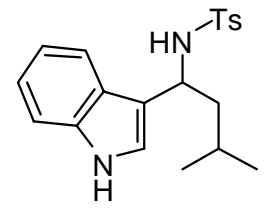

Racemic 3Ak

(-)-3Ak 95\% ee

Product obtained with 7

(+)-3Ak 95\% ee

Product obtained with 6
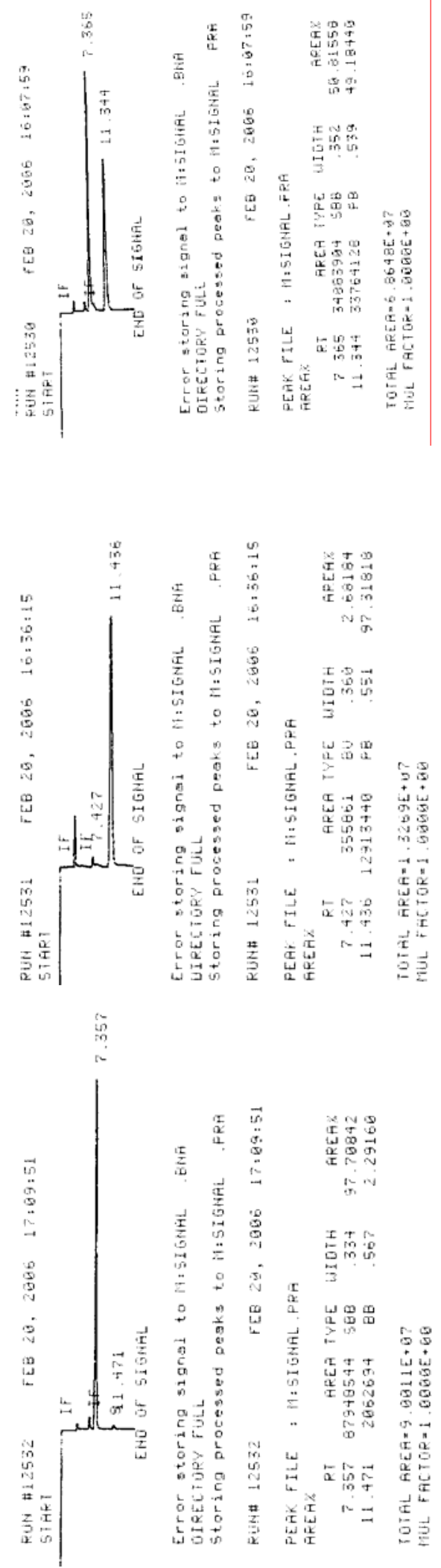
HPLC Conditions: Daicel chiralpak OD, Hexanes:IPA, 80:20 1.0 mL/min, $\lambda 220 \mathrm{~nm}$

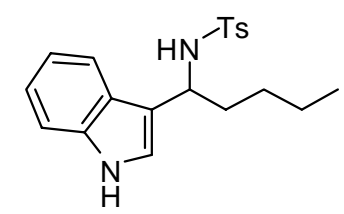

Racemic 3Al
(-)-3Al 96\% ee

Product obtained with 7

(+)-3Al 97\% ee

Product obtained with 6
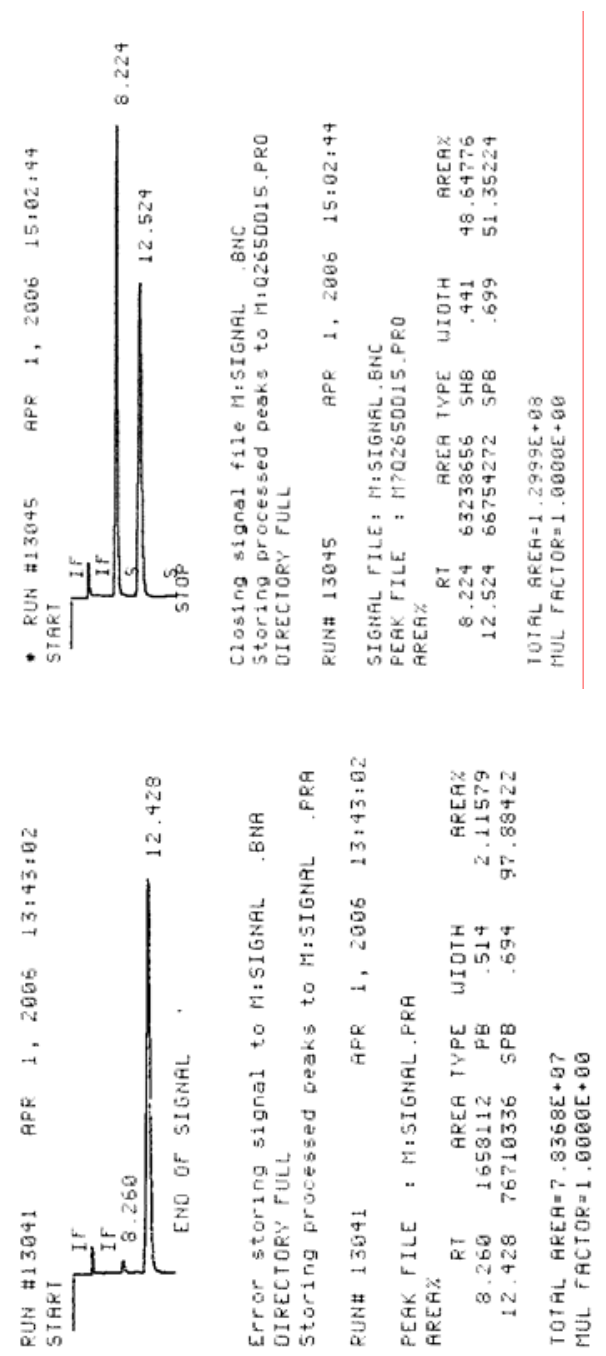

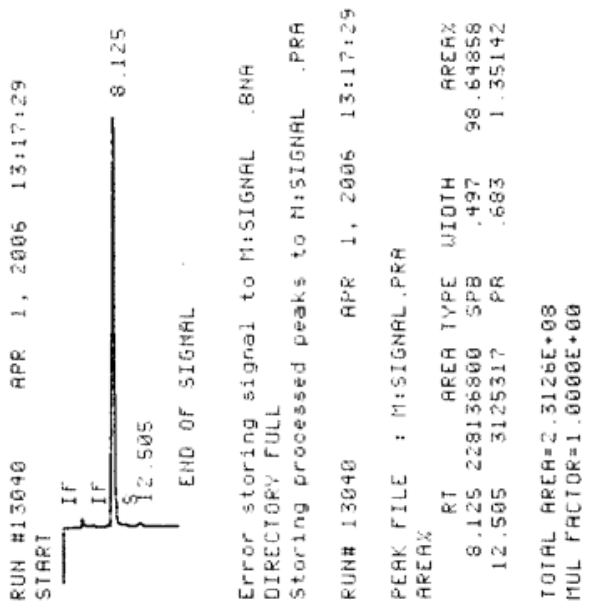


HPLC Conditions: Daicel chiralpak OD, Hexanes:IPA, 80:20 1.0 mL/min, $\lambda 220 \mathrm{~nm}$

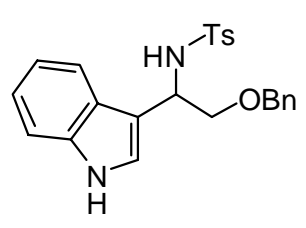

Racemic 3Am

(-)-3Am 96\% ee

Product obtained with 7

(+)-3Am 96\% ee

Product obtained with 6
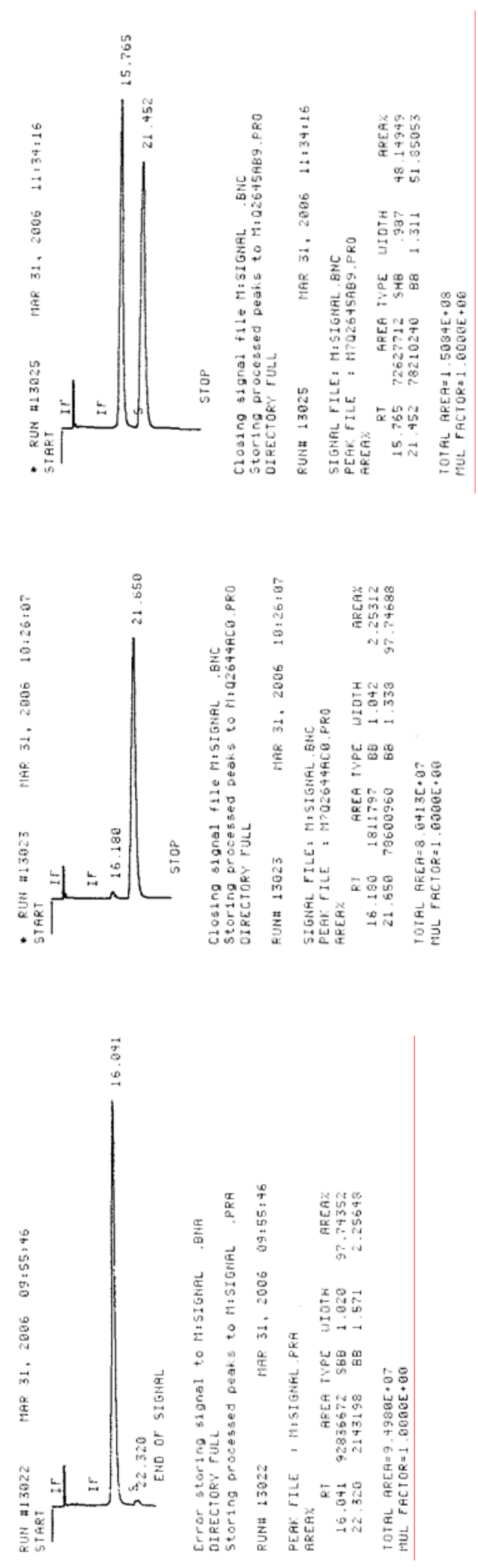
HPLC Conditions: Daicel chiralpak OD, Hexanes:IPA, 60:40 1.0 mL/min, $\lambda 220 \mathrm{~nm}$<smiles>O=C(NC(c1ccccc1)c1c[nH]c2ccccc12)OCc1ccccc1</smiles>

Racemic 8Ab

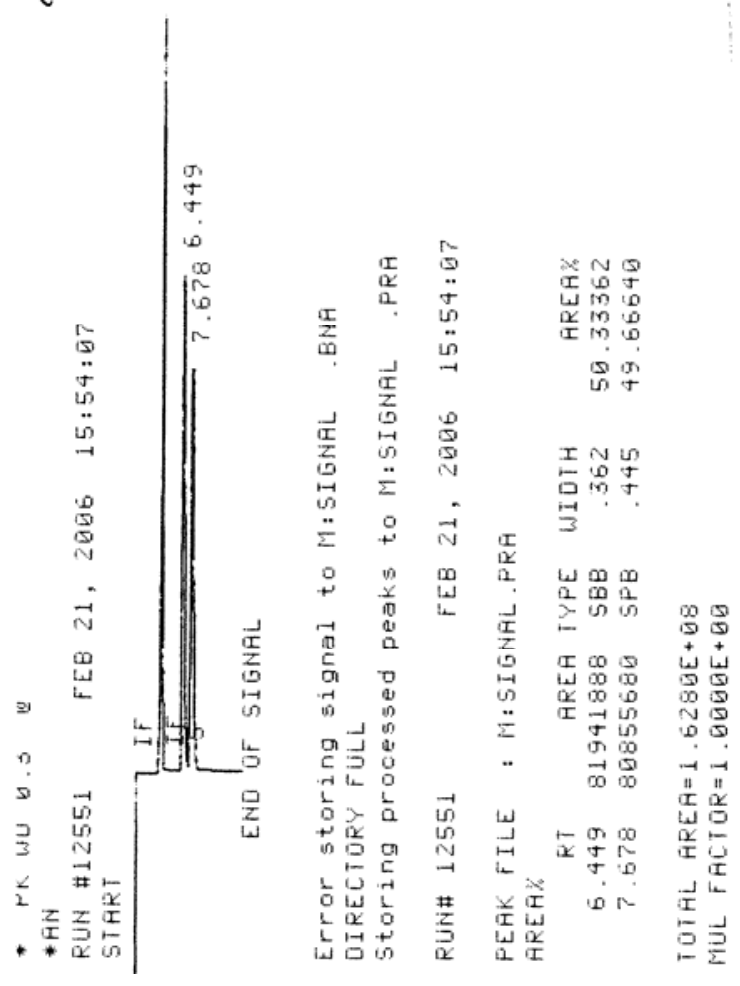

8Ab, 94\%ee

Product obtained from $(+)-\mathbf{3 A b}$

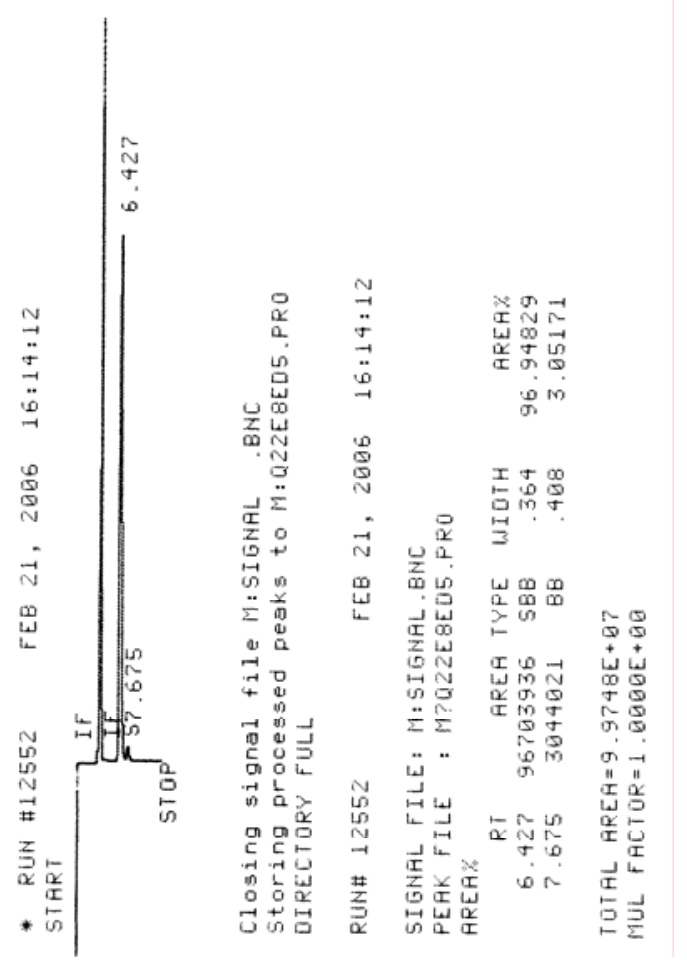


HPLC Conditions: Daicel chiralpak OD, Hexanes:IPA, 70:30 1.0 mL/min, $\lambda 220 \mathrm{~nm}$

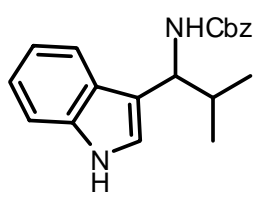

Racemic 8Aj

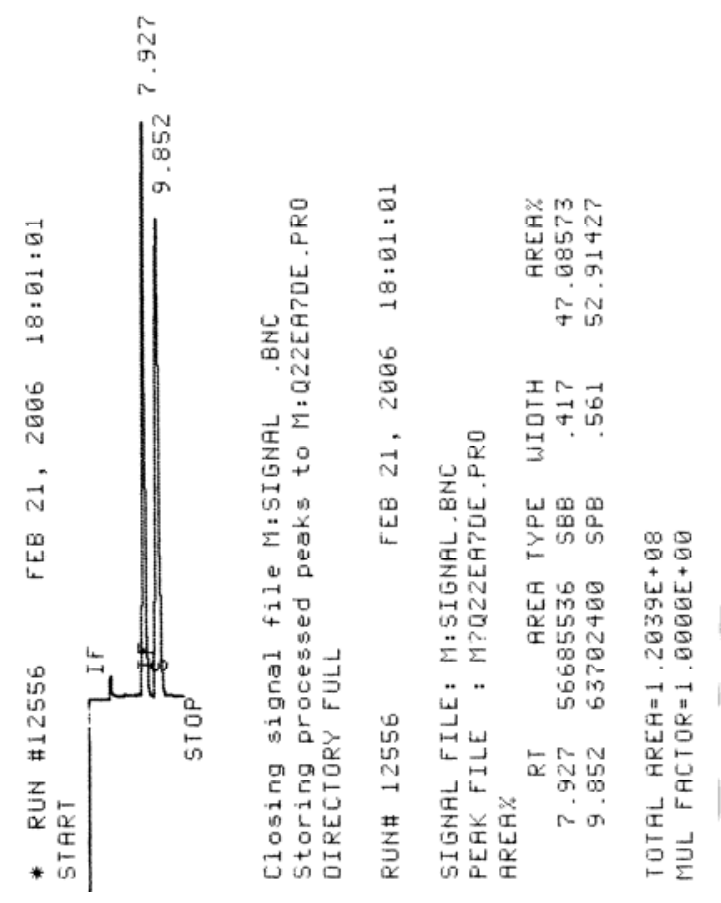

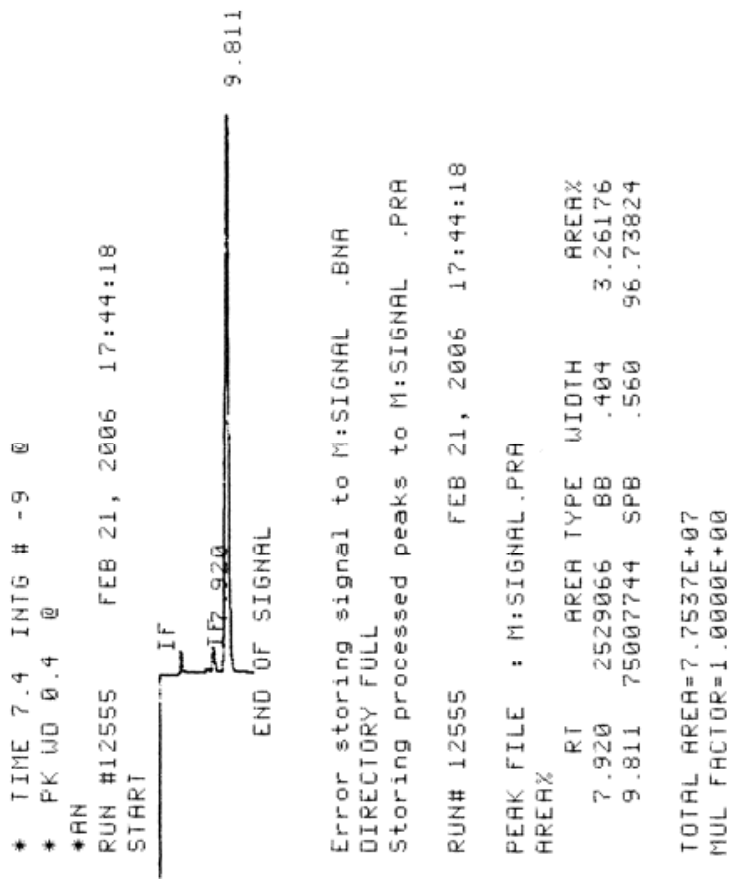

8Aj, 94\%ee

Product obtained from (-)-3Aj 

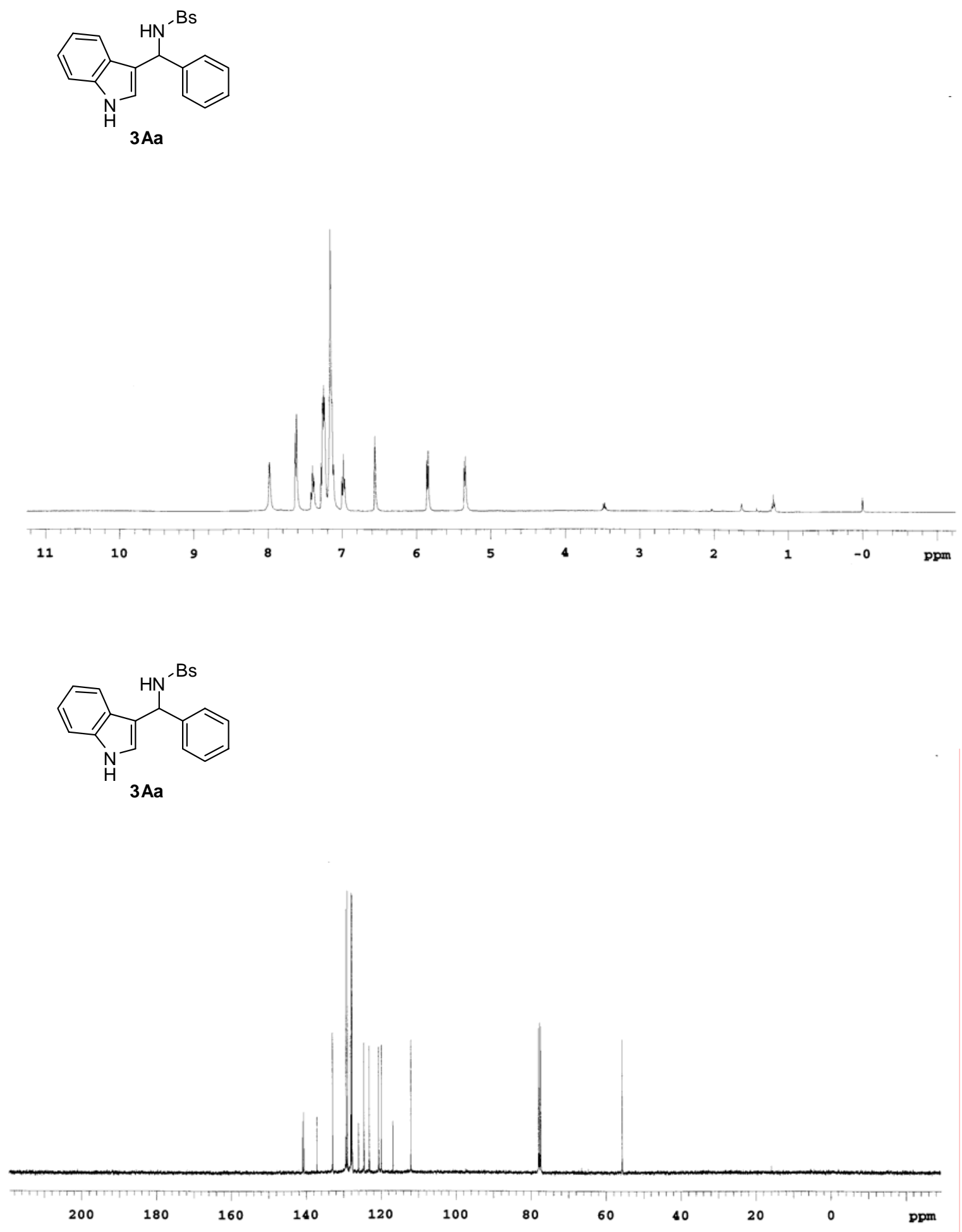

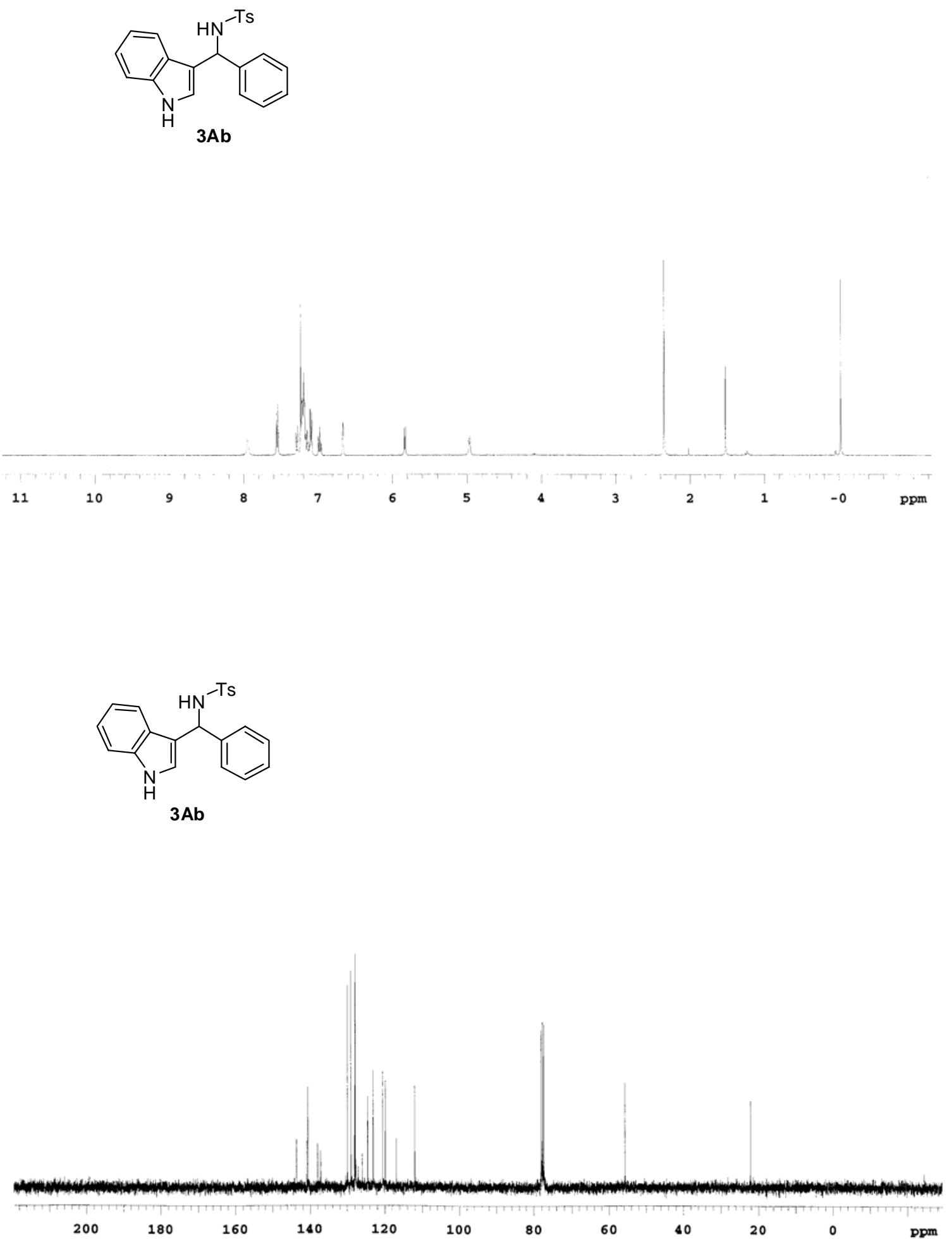

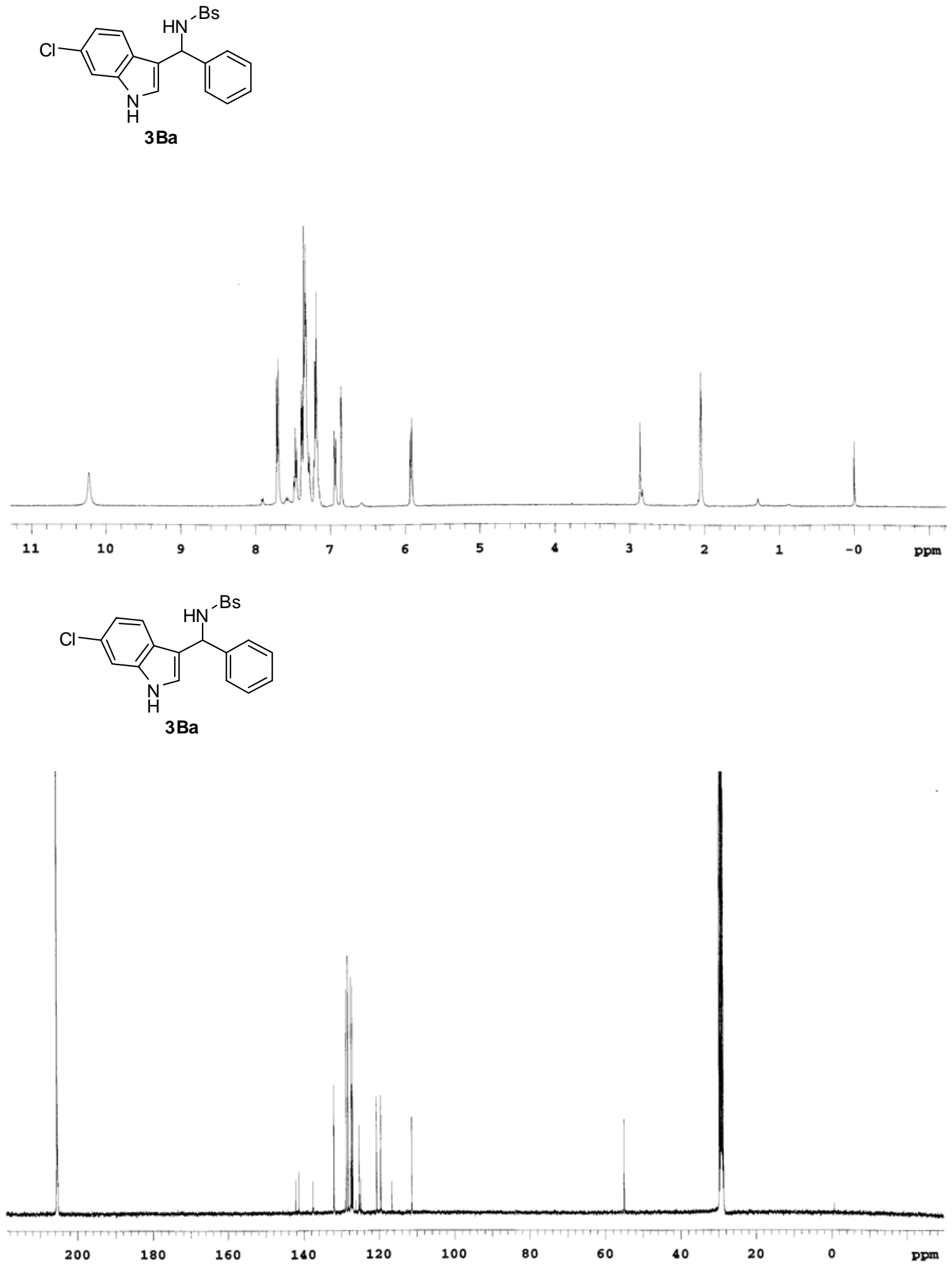

S-49 

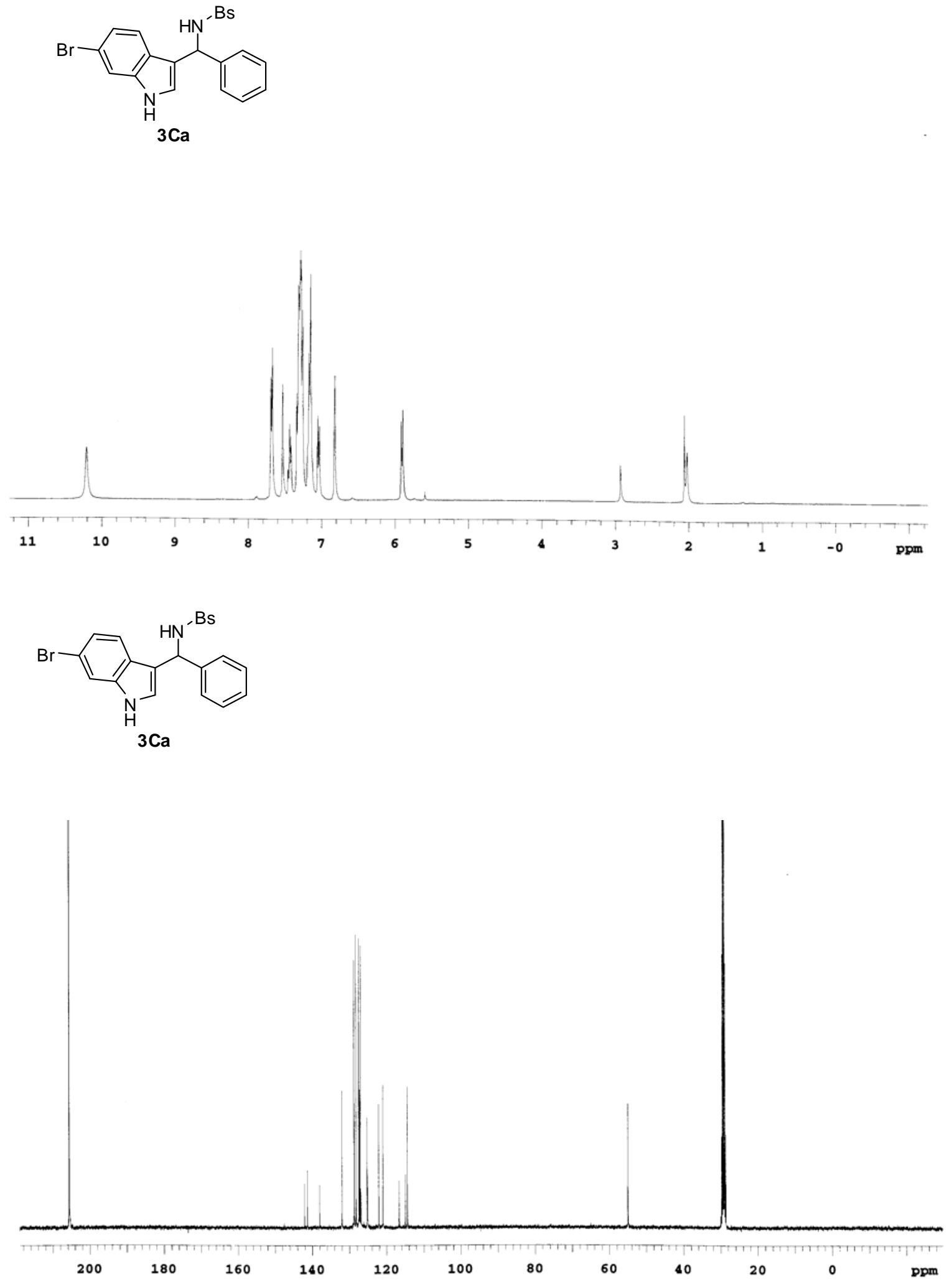

S-50 

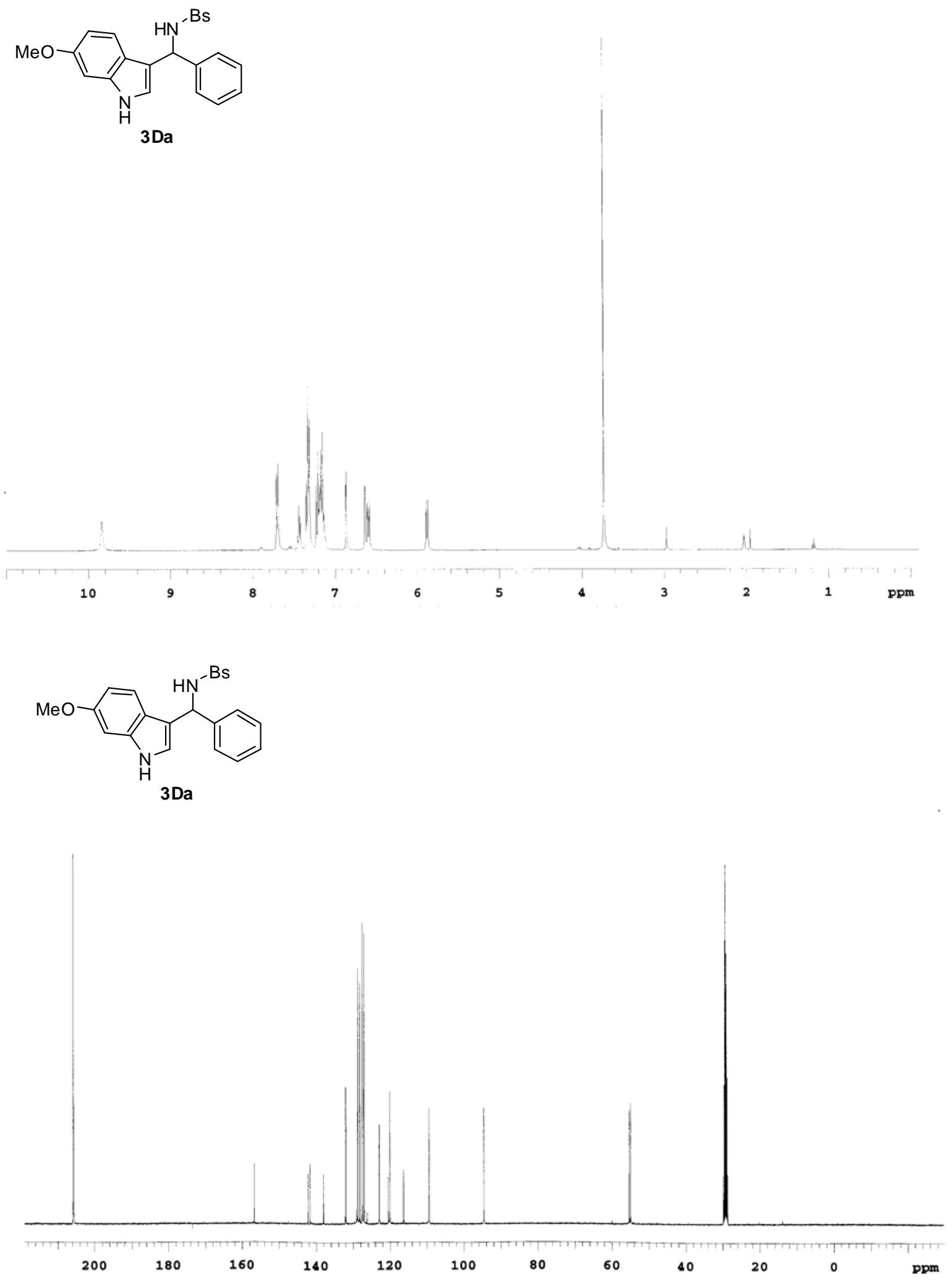

S-51 

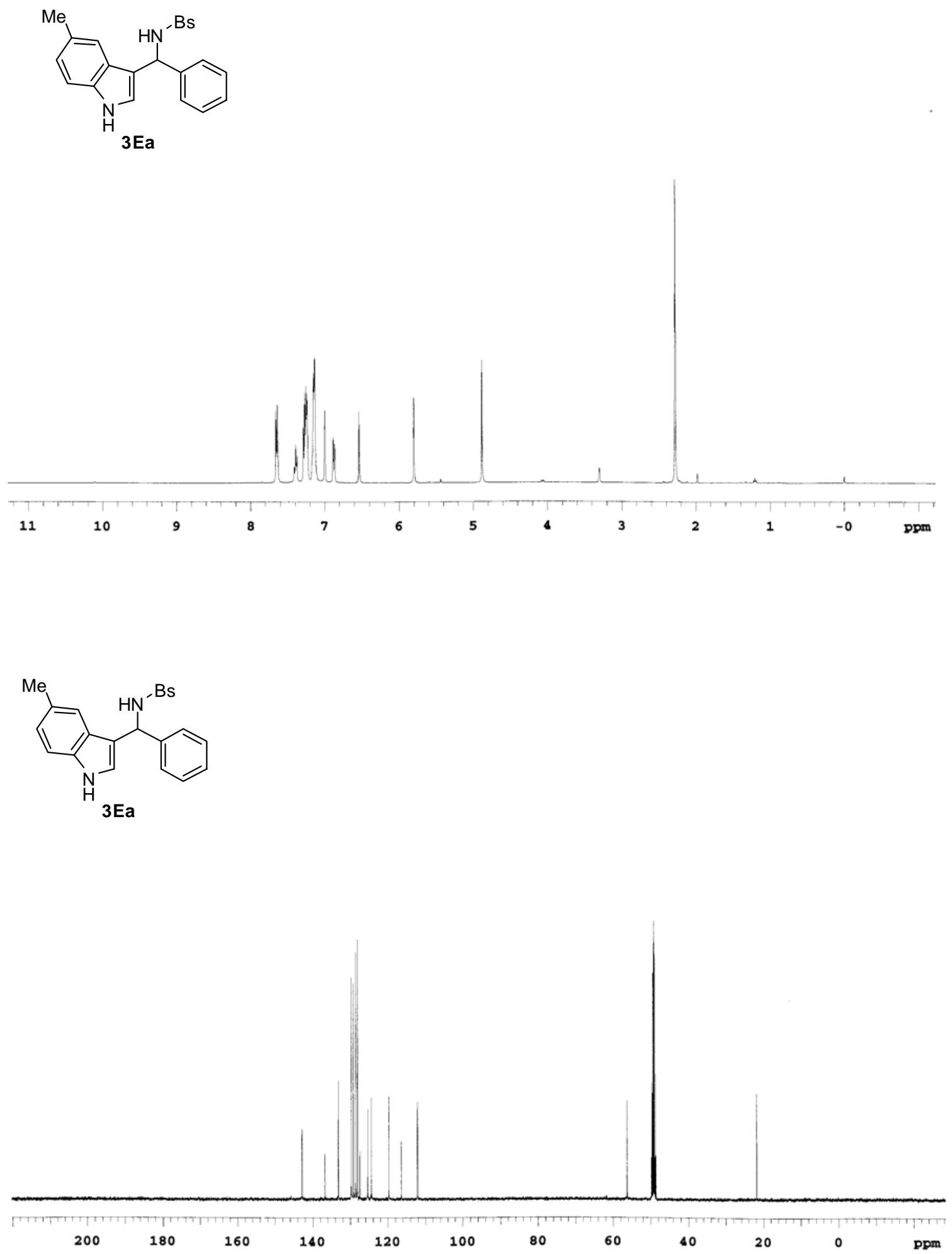


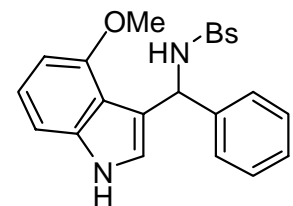

3Fa

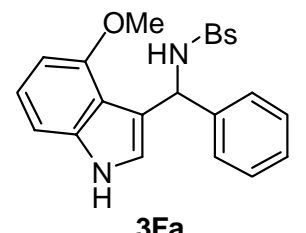

3Fa

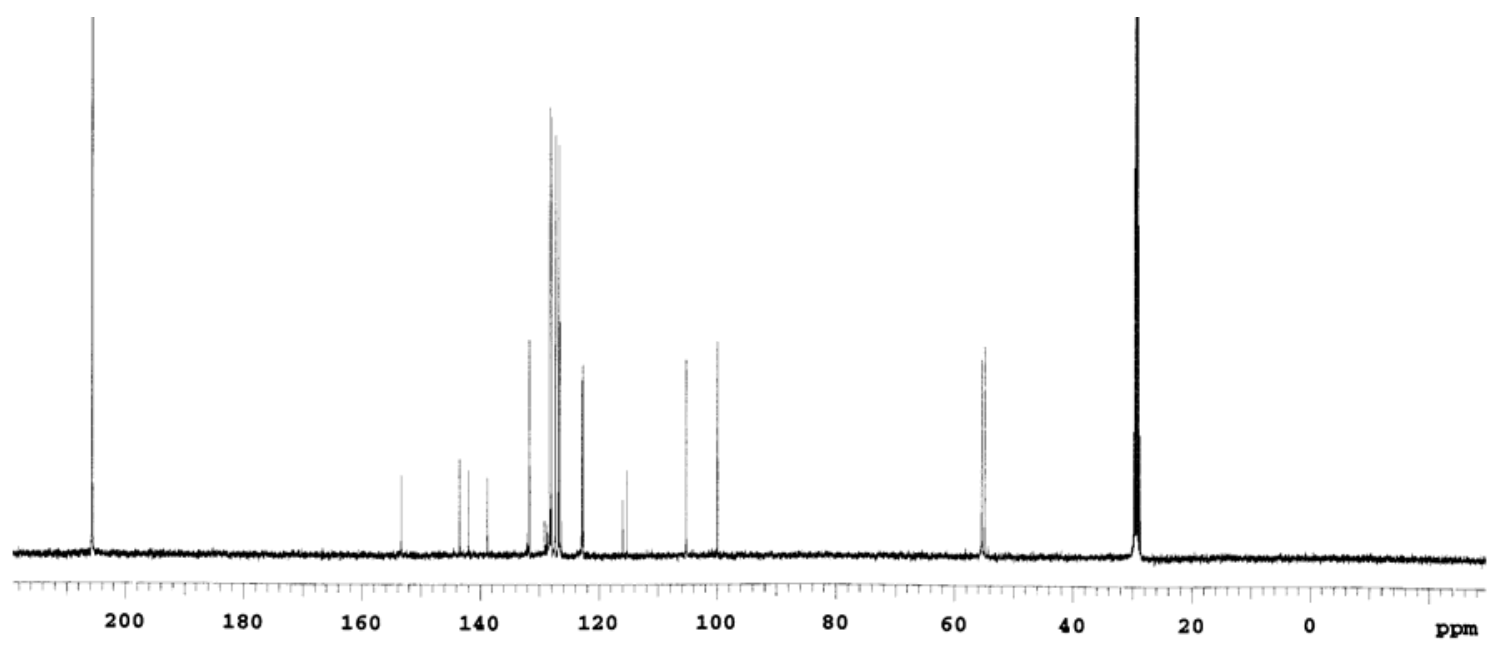



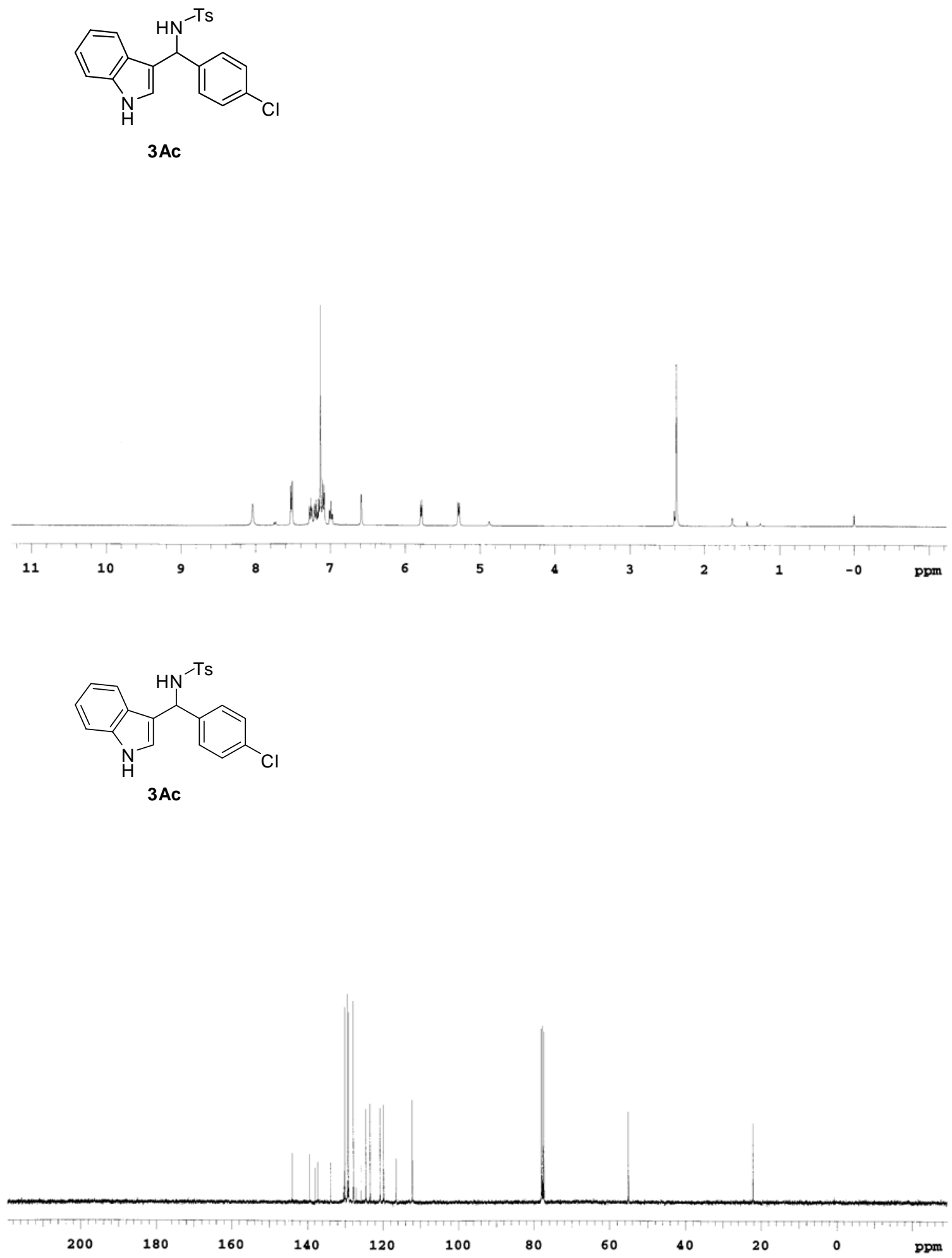

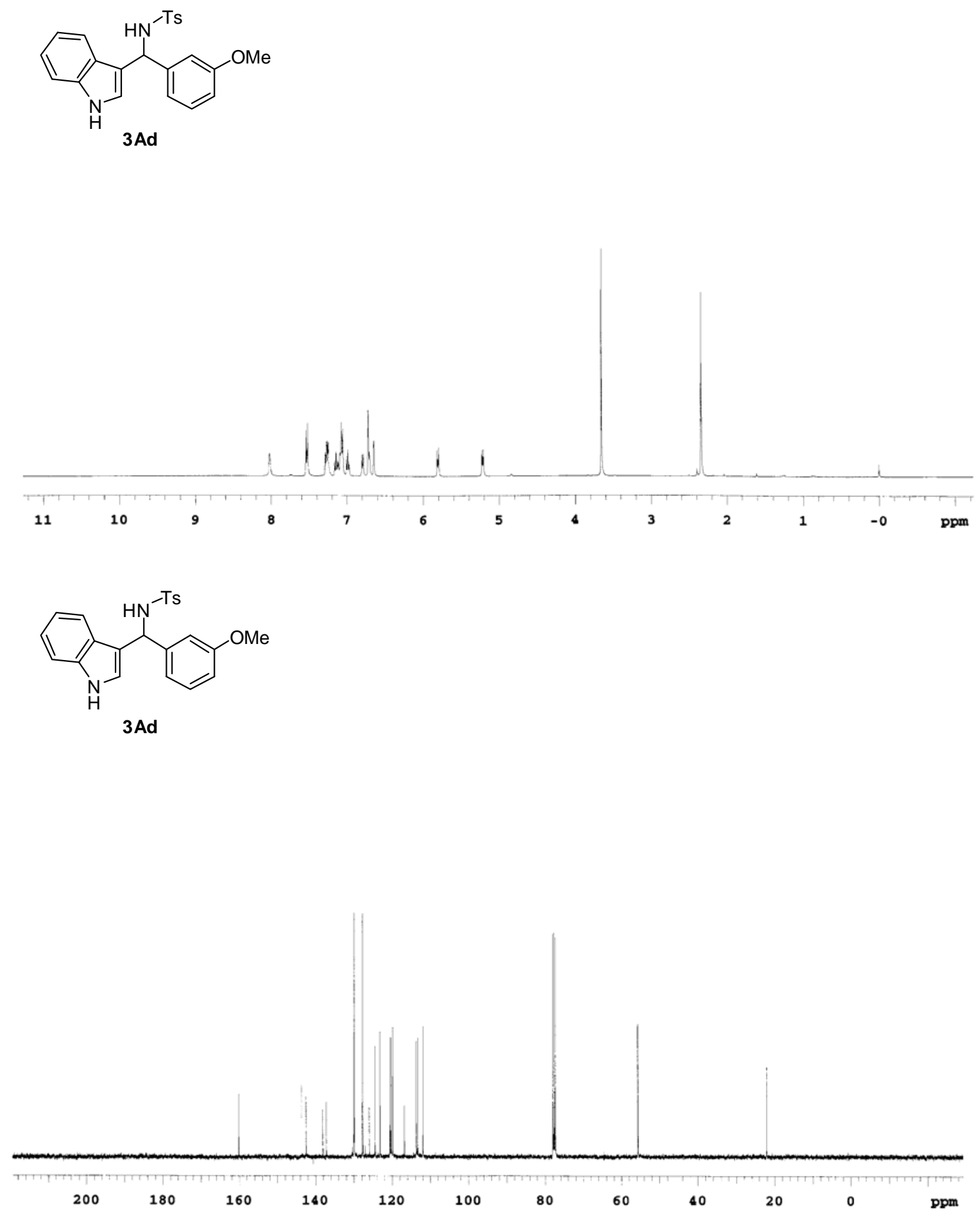

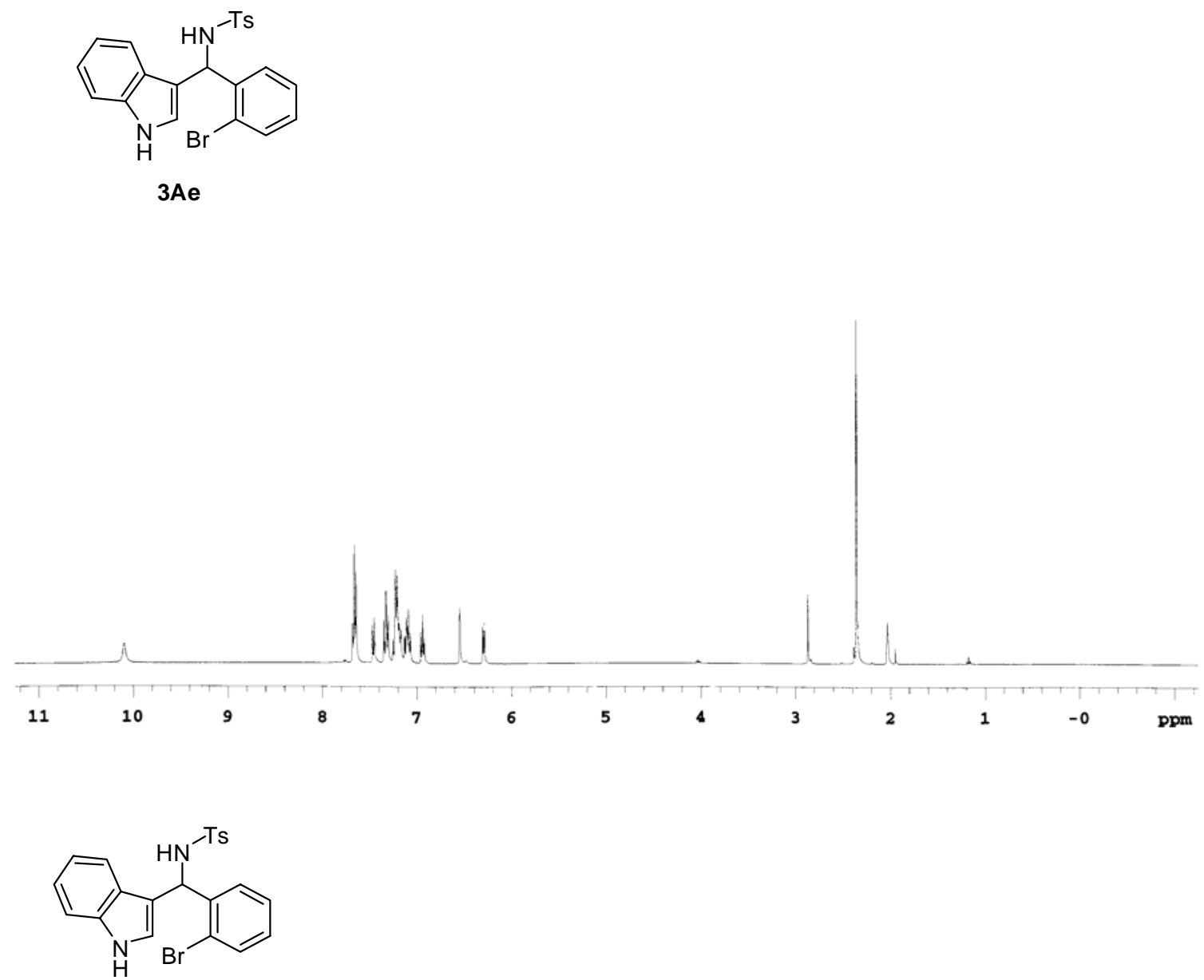

3Ae

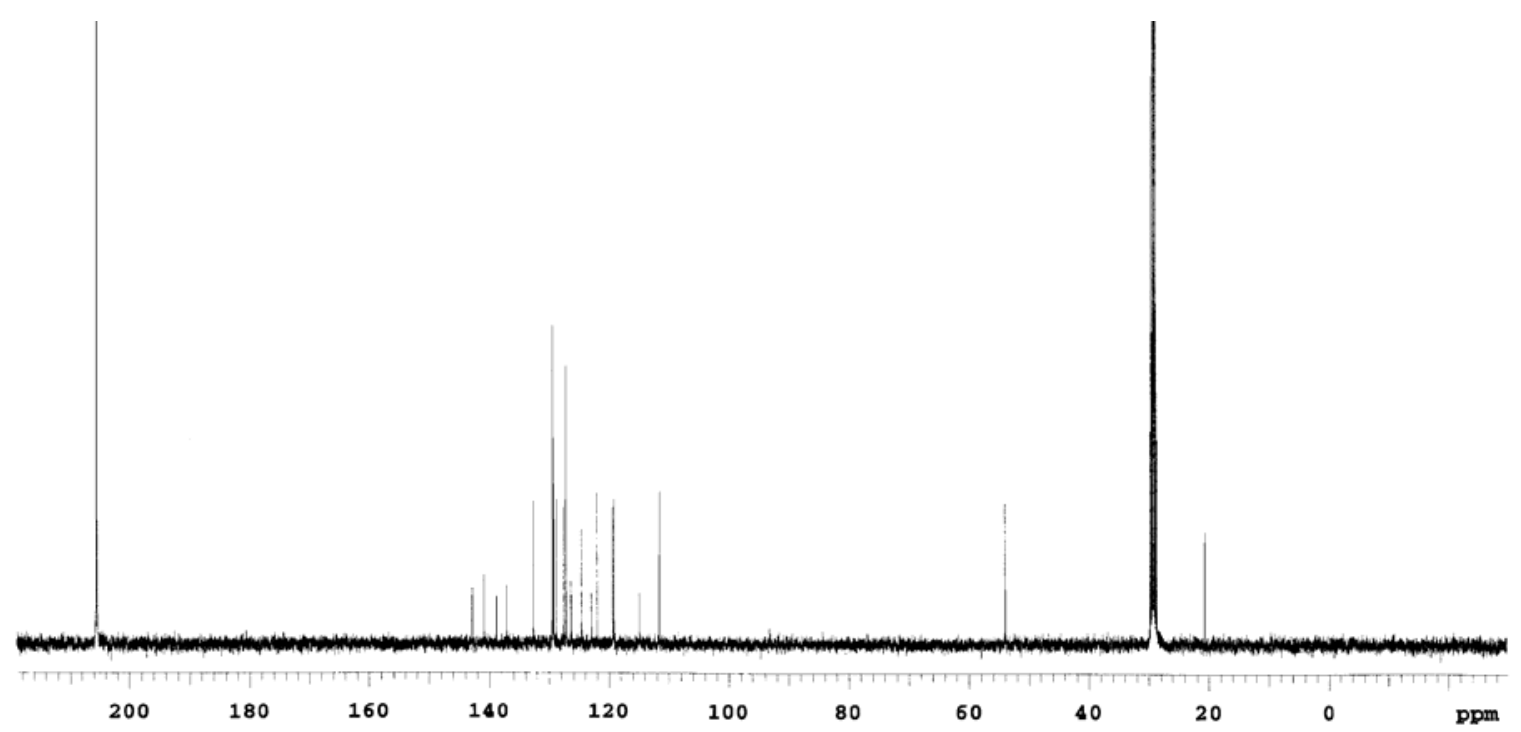



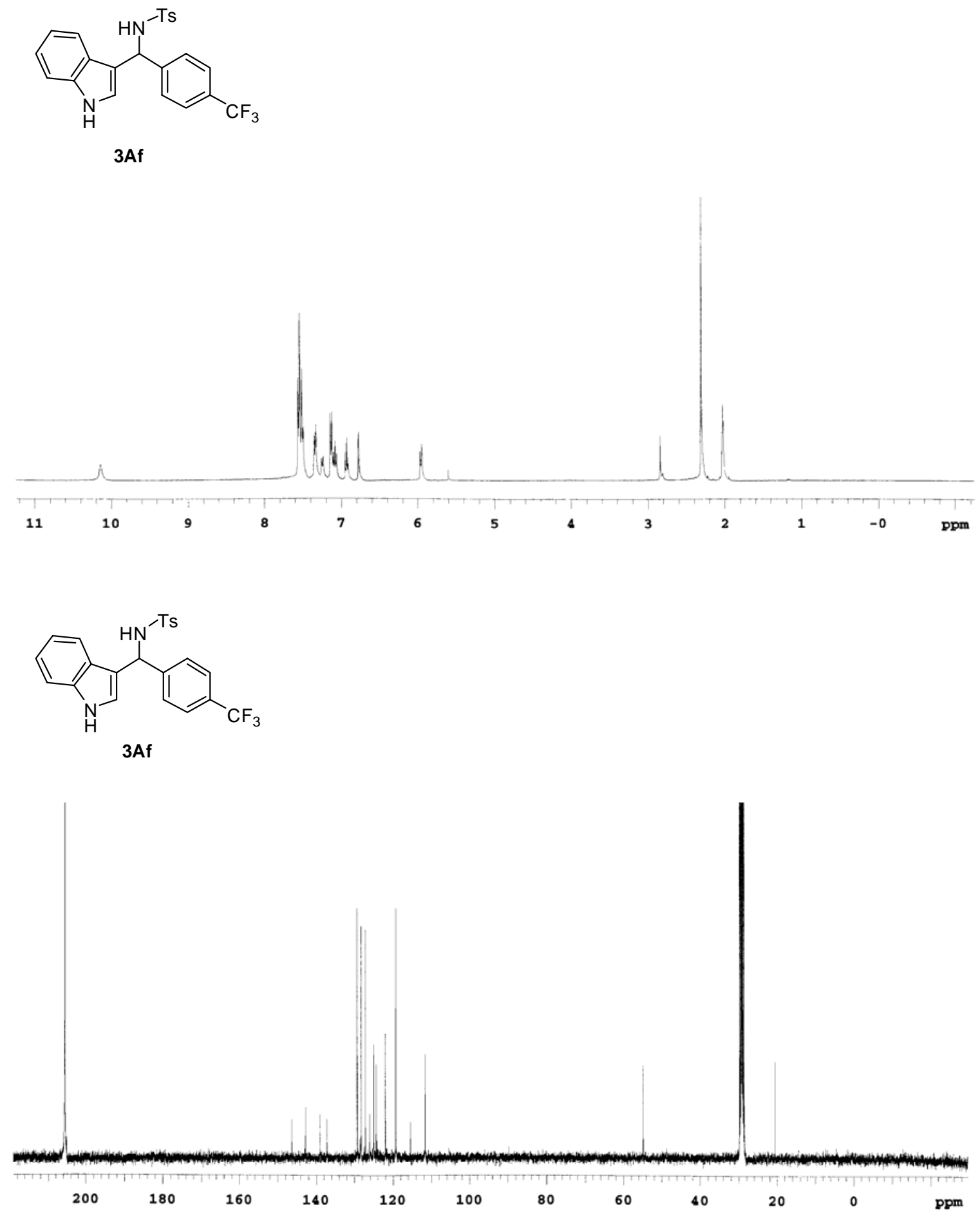

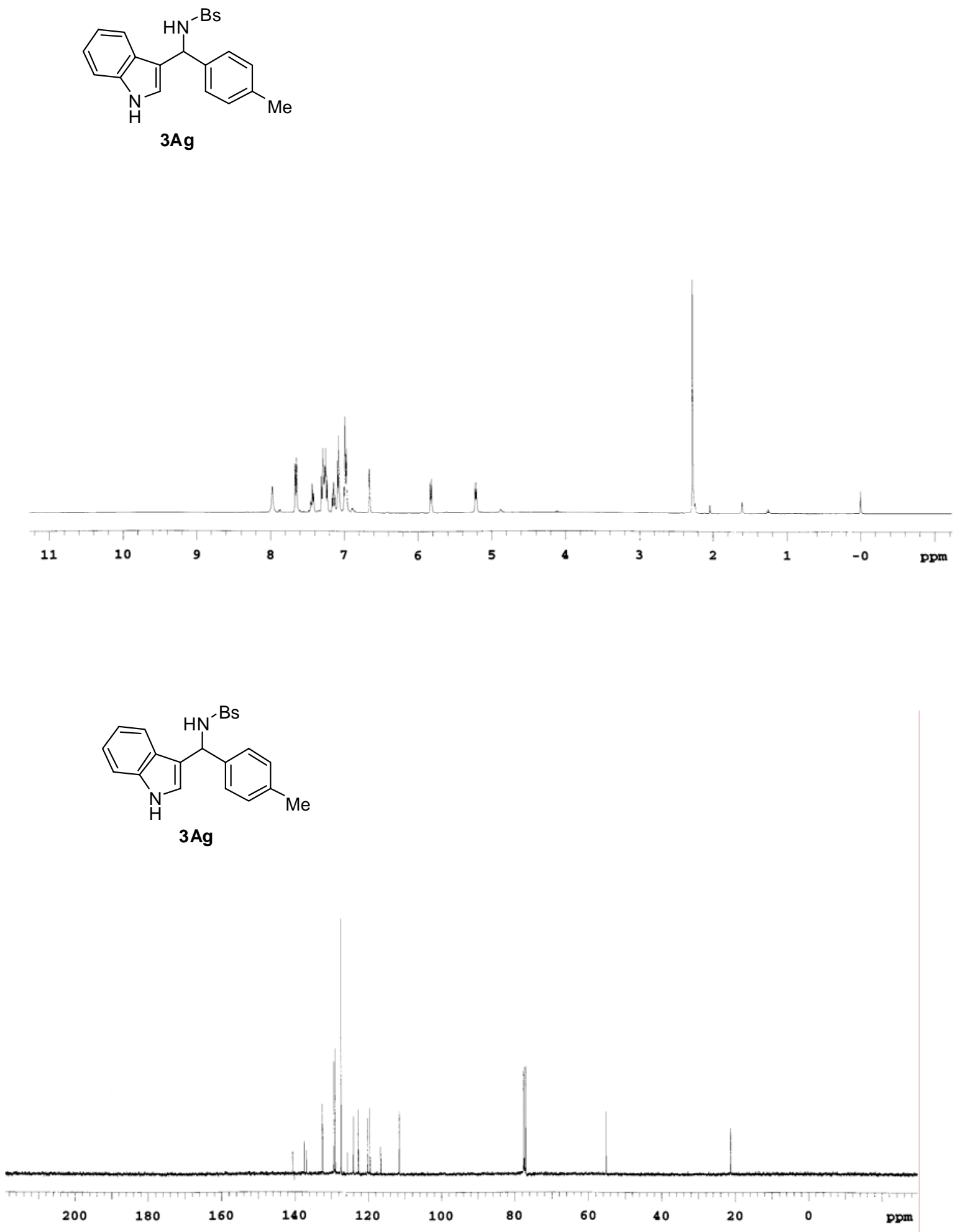

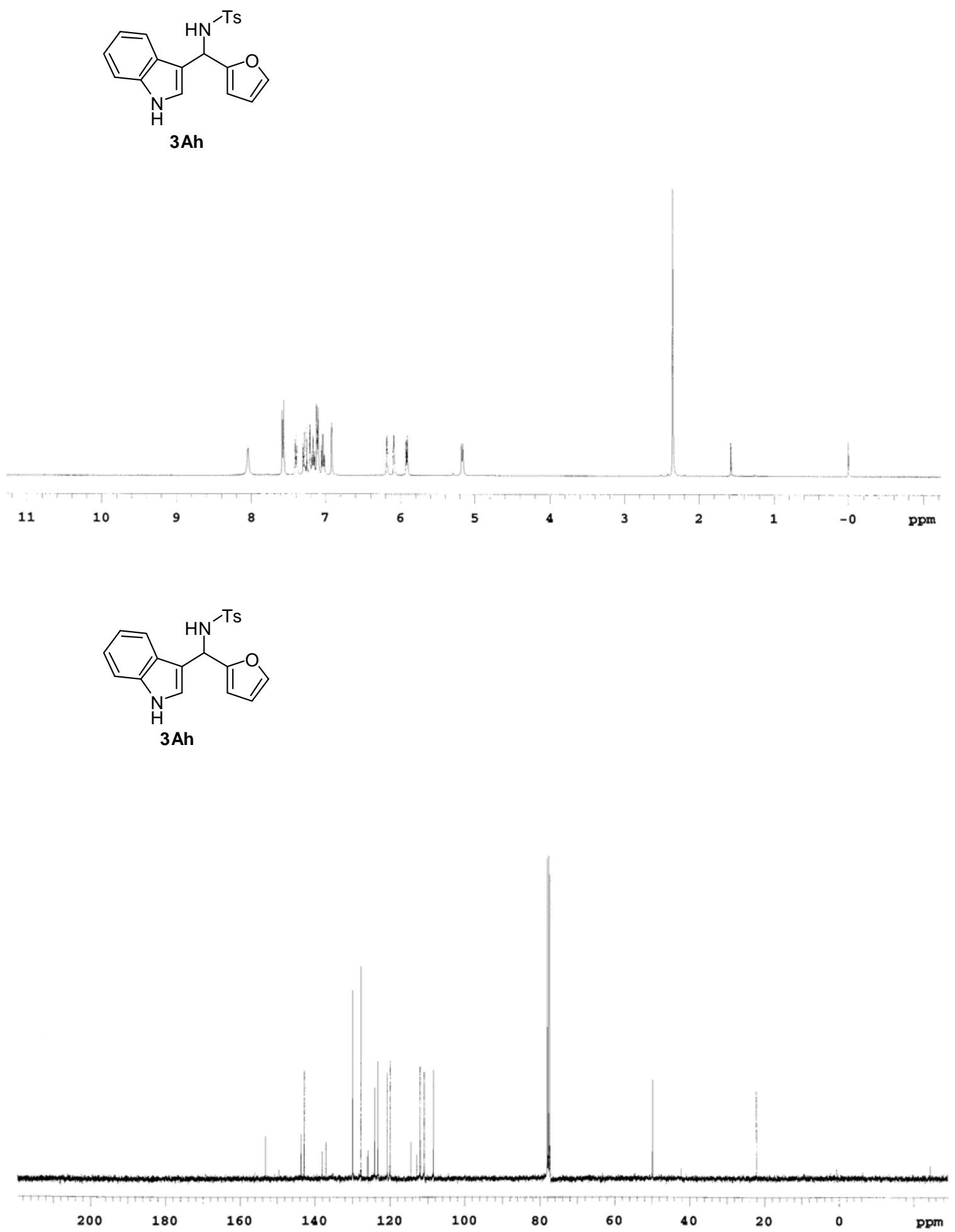

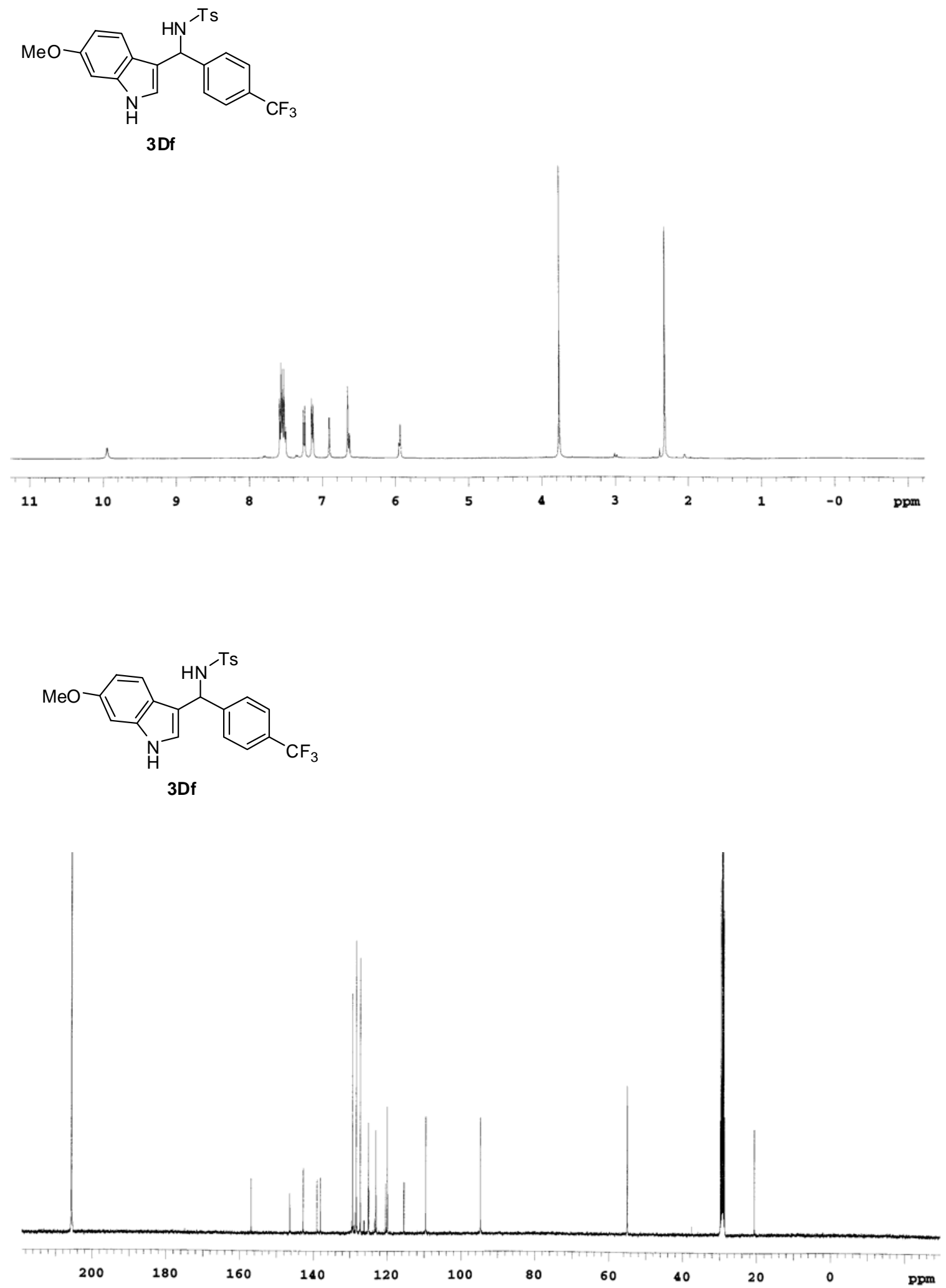

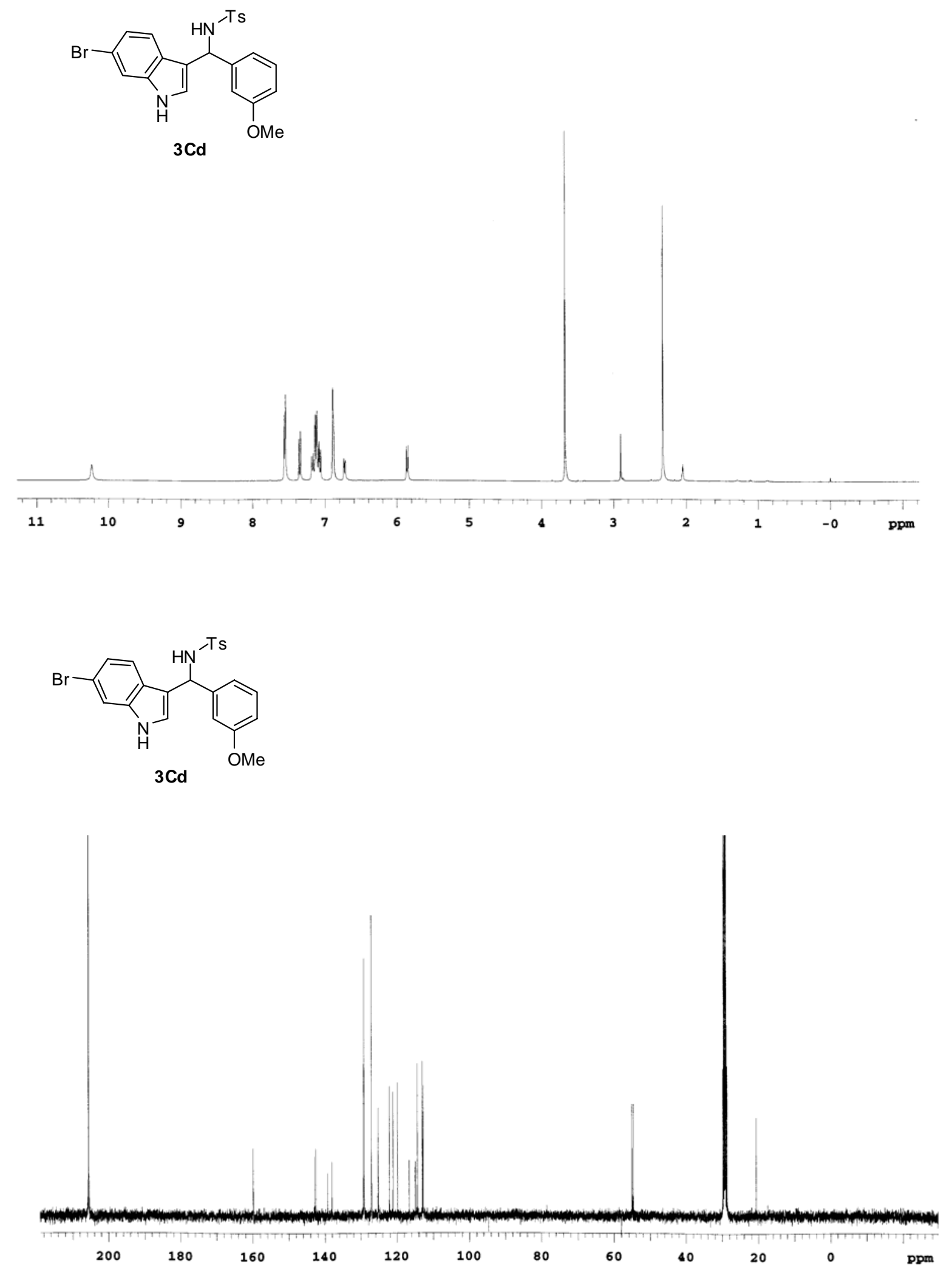

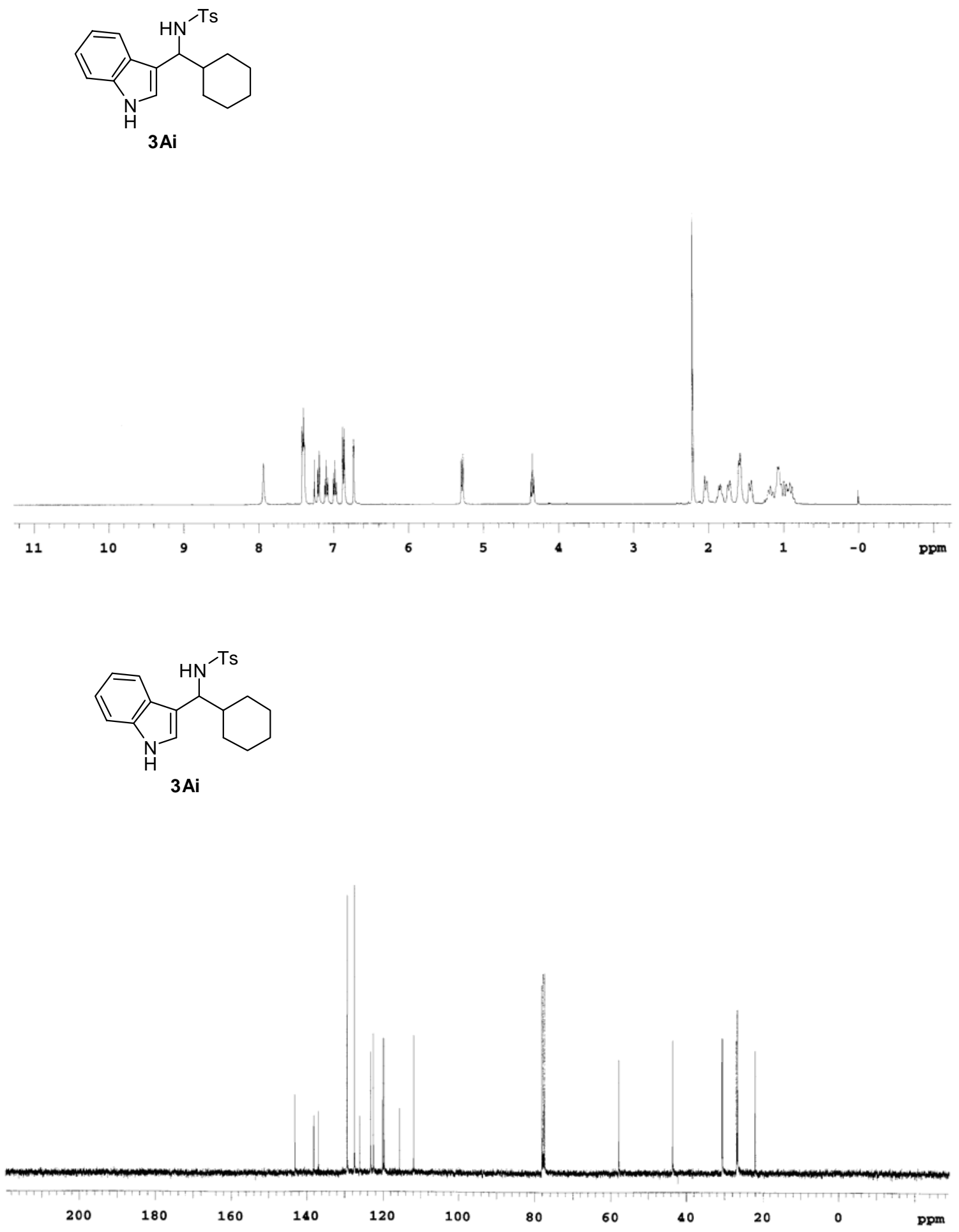

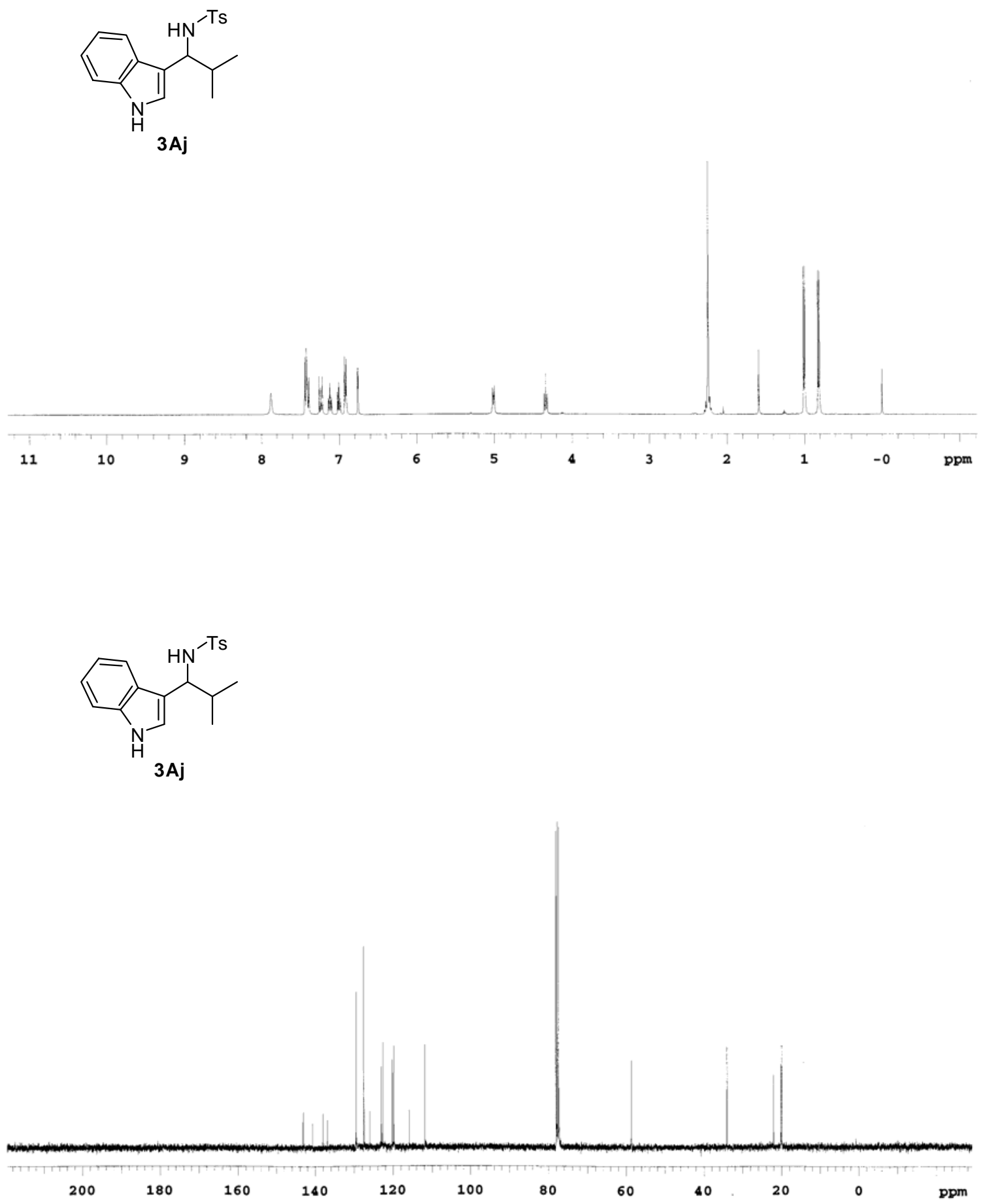

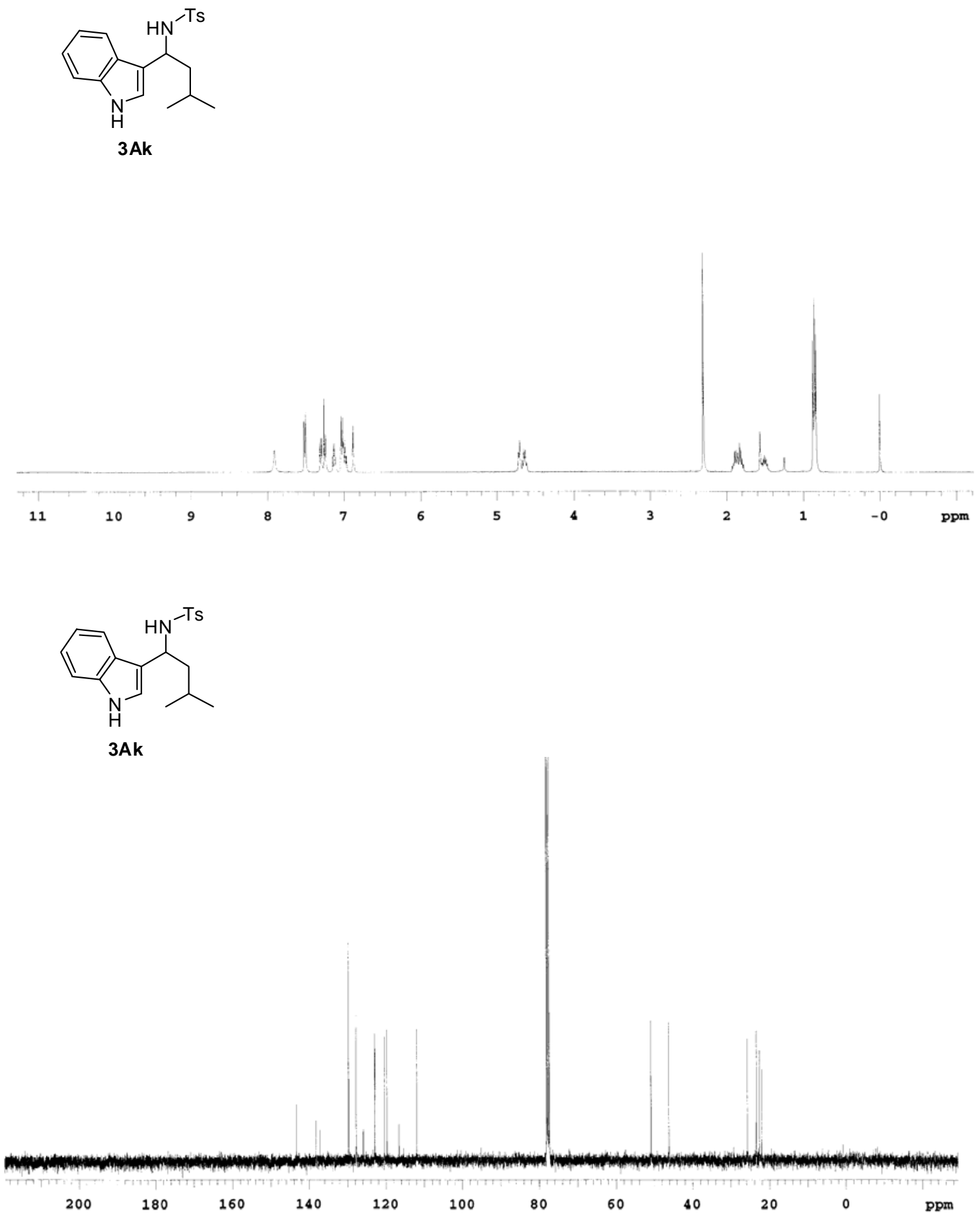

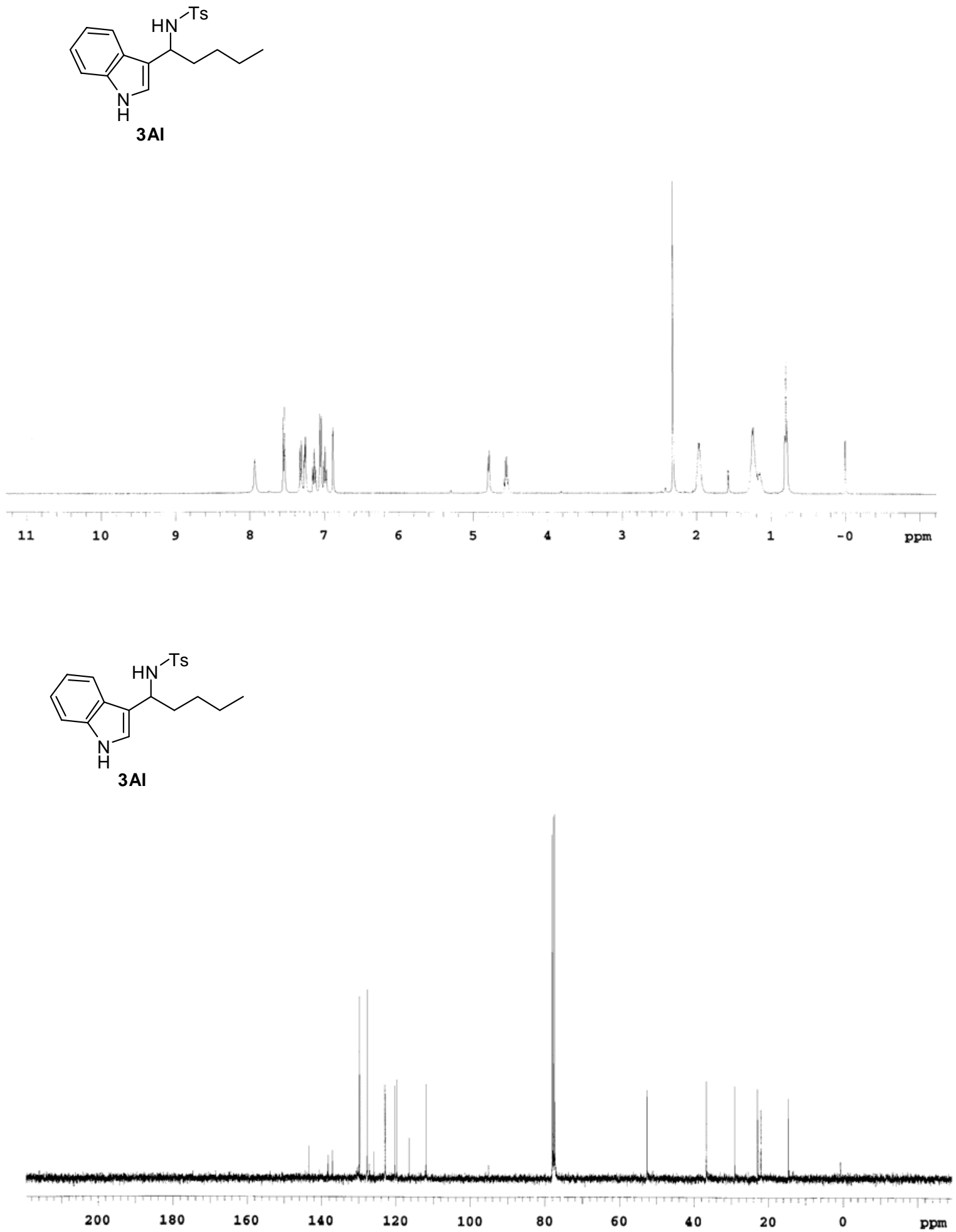

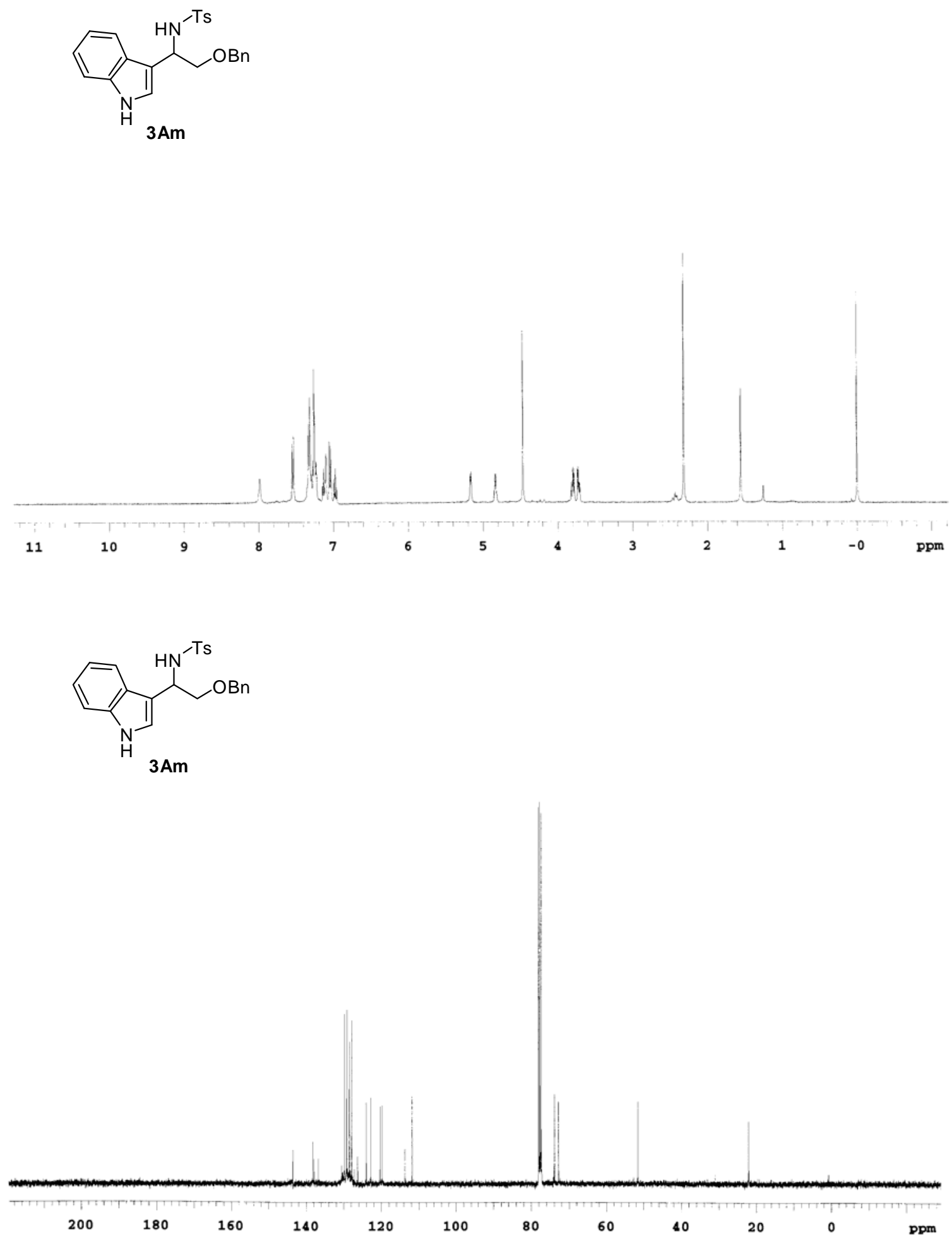

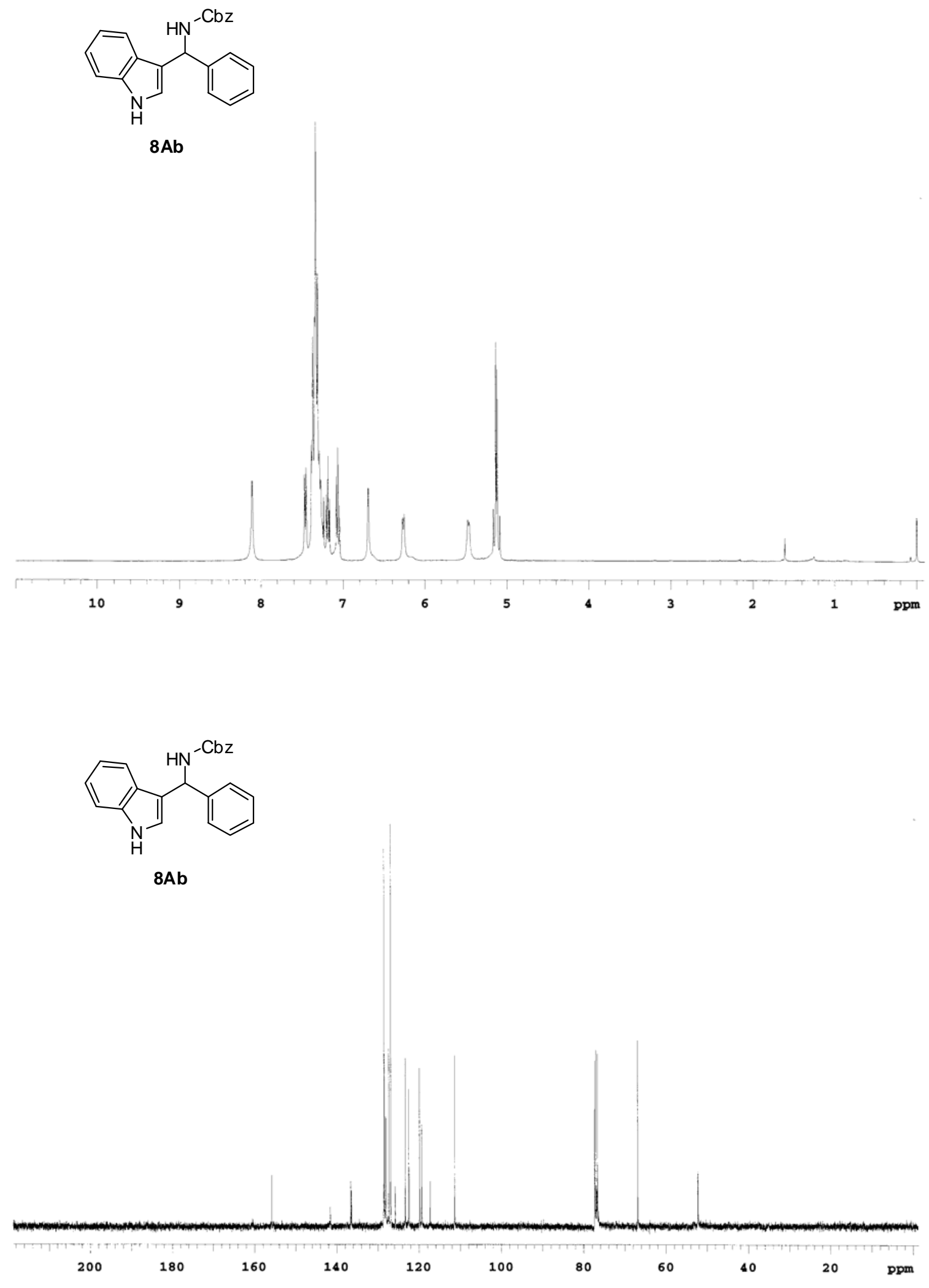

S-67 

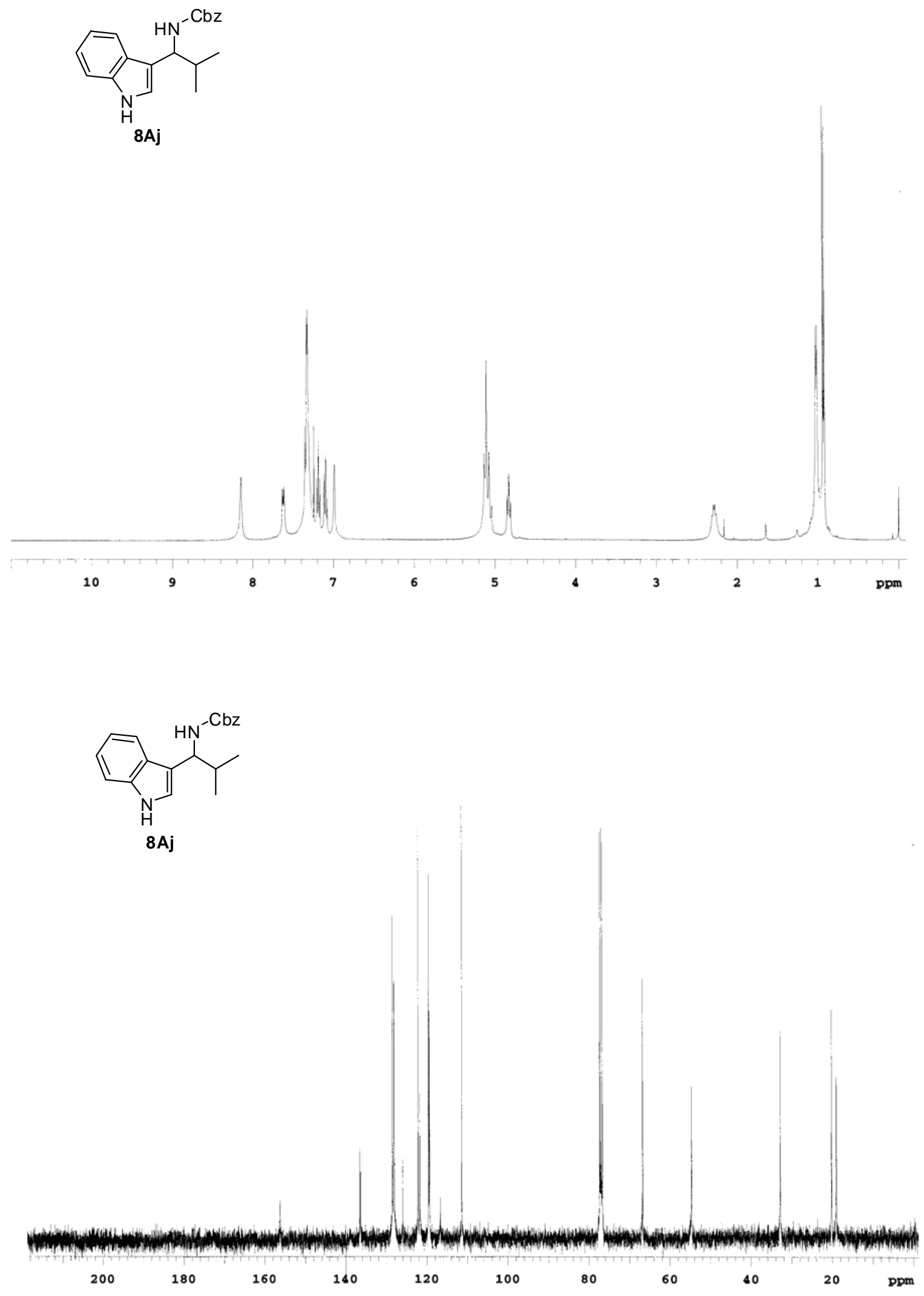

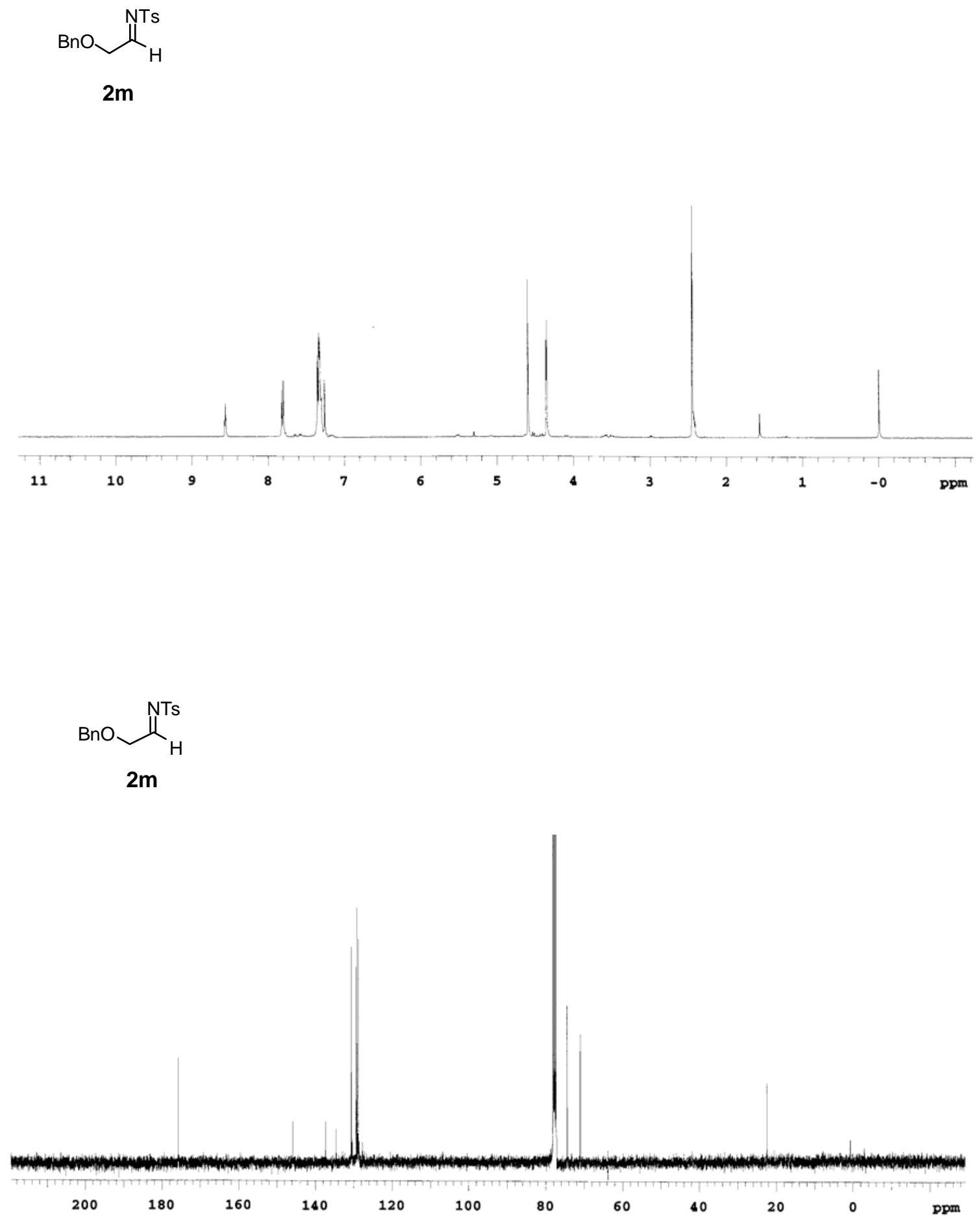